\title{
LAGRANGE'S THEORY OF ANALYTICAL FUNCTIONS AND HIS IDEAL OF PURITY OF METHOD
}

\author{
GIOVANNI FERRARO AND MARCO PANZA
}

\begin{abstract}
We reconstruct essential features of Lagrange's theory of analytical functions by exhibiting its structure and basic assumptions, as well as its main shortcomings. We explain Lagrange's notions of function and algebraic quantity, and concentrate on power-series expansions, on the algorithm for derivative functions, and the remainder theorem-especially the role this theorem has in solving geometric and mechanical problems. We thus aim to provide a better understanding of Enlightenment mathematics and to show that the foundations of mathematics did not, for Lagrange, concern the solidity of its ultimate bases, but rather purity of method - the generality and internal organization of the discipline.
\end{abstract}

\section{Preliminaries and Proposals}

Foundation of mathematics was a crucial topic for 18th-century mathematicians. A pivotal aspect of it was the interpretation of the algoritihms of the calculus. This was often referred to as the question of the "metaphysics of the calculus" (see Carnot 1797, as an example).

Around 1800 Lagrange devoted two large treatises to the matter, both of which went through two editions in Lagrange's lifetime: the Théorie des fonctions analytiques (Lagrange 1797, 1813; henceforth the Théorie); and the Leçons sur le calcul des fonctions (Lagrange 1797, 1813; henceforth the Leçons). His aim was to provide a new and non-infinitesimalist interpretation of these algorithms based on a general theory of power series. ${ }^{2}$ He viewed the direct algorithm as a rule for transforming functions, which-applied reiteratively to any function $y=f(x)$ - gives, apart from numerical factors, the coefficients of the expansion of $f(x+\xi)$ in a power series of the indeterminate increment $\xi .{ }^{3}$ Lagrange called such coefficients 'derivative functions [fonctions dérivées]' (Lagrange 1797, art. 17; 1801, p. 5; 1806, p. 5; 1813, Introduction, p. 2): a term whose meaning has since changed. In what follows, we shall use this term in Lagrange's sense.

Throughout his theory, Lagrange certainly pursues an ideal of conceptual clarity involving the elimination of any sort of infinitesimalist insight. This has been often noticed, and is emphasized by Lagrange himself from the very complete title of the Théorie: Théorie des fonctions analytiques, contenant les principes du calcul différentiel, dégagés de toute considération d'infiniment petits, d'evanouissans, de limites et de fluxions, et réduite à l'analyse algébrique des quantités finies. We shall not dwell on this point, then. We shall rather argue that this ideal was part of a more general ideal of purity of method: ${ }^{4}$ the reduction of all mathematics to an algebraic, purely formal theory ${ }^{5}$ centered of the manipulation of (finite or infinite) polynomials through the method of indeterminate coefficients. ${ }^{6}$

This was a sweeping project rooted in a mathematical program going back to the early mathematical work of Newton (see Panza 2005), and whose manifesto was the first volume of Euler's Introductio in analysin infinitorum (Euler 1748). ${ }^{7}$ Its main purpose was the development of a fairly general and formal theory of abstract quantities: quantities merely conceived as elements of a net of relations, expressed by formulas belonging to an appropriate language and subject to appropriate transformation rules.

\footnotetext{
${ }^{1}$ We use double inverted commas for quotation and simple ones for mention. We never use inverted commas for other purposes, namely for emphasizing a term or phrase.

${ }^{2}$ A few years earlier, Arbogast had proposed a similar interpretation in an unpublished treatise (Arbogast ESSAI; for a commentary, see: Zimmermann 1934; Panza 1985; Grabiner 1990, pp. 47-59). According to Grabiner (1981b, p. 316), this treatise was inspired by an even earlier paper by Lagrange himself (Lagrange 1772; see footnote 3.2, below). Lagrange mentions Arbogast's treatise at the beginning of the Théorie (Lagrange 1797, art. 7; 1813, Introduction, p. 5).

${ }^{3}$ Some clearly indicated exceptions apart, we shall use the term 'power-series expansion of $f(x+\xi)$ ' for referring to the expansion of $f(x+\xi)$ in a power series of $\xi$. Lagrange uses ' $\xi$ ' to denote the increment of $x$ in his earlier paper mentioned in footnote 2 . In the Théorie and the Leçon, he uses the Latin letter ' $i$ ' (as 'incrément')—but we prefer using ' $\xi$ ' in order to avoid any possible confusion with the symbol that is now used to denote $\sqrt{-1}$. Our quotations from Lagrange's treatises are altered accordingly.

${ }^{4}$ On the notion of purity of method, see Arana (2008), Detlefsen (2008)—which explicitly mentions Lagrange's "purification program", in footnote 6, p. 182-, and Hallett (2008). In this last paper (ibid., p. 199), M. Hallet describes as follows the concern for purity of method , by referring to Hilbert's mention of such a concern in Hilbert (1899, p. 199): "one can enquire of a given proof or of a given mathematical development whether or not the means it uses are 'appropriate' to the subject matter, whether one way of doing things is 'right', whereas another, equivalent way is 'improper'."

${ }^{5}$ The clarification of the exact sense in which the adjectives 'algebraic' and 'formal' have to be understood here is one of the main purposes of our paper.

${ }^{6}$ The centrality of the method of indeterminate coefficients is the common denominator of Lagrange's foundational programs both in pure mathematics and in mechanics. For the case of mechanics, see Panza (1991-1992) and (2003).

${ }^{7}$ An attempt to reconstruct the sources and evolution of this program up to Lagrange is made in Panza (1992). The present paper is partly based on chapter III.6.
} 
The interpretation of the calculus and its algorithms presented a crucial difficulty for the complete accomplishment of such a program. The difficulty was not only concerned with the conceptual inaccuracy of the current infinitesimalist or pseudo-infinitesimalist accounts. Above all, it was their reliance on suppositions-like the negligibility of infinitesimalsthat could hardly be explained in purely formal terms. The following passage, from a short report by Lagrange himself, clearly illustrates the point:

[...] I do not deny that one could rigorously prove the principles of the differential calculus through the consideration of limits envisaged in a particular way, as Maclaurin, d'Alembert and several others after them did. But the kind of metaphysics that has to be applied for this purpose is, if not contrary, at least foreign to the spirit of analysis, which should have no metaphysics but that which consists in the first principles and in the fundamental operations of calculation.

$[\ldots]$ je ne disconviens pas qu'on ne puisse, par le considérations des limites envisagées d'une manière particulière, démontrer rigouresement les principes du calcul différentiel, comme Maclaurin, d'Alembert et plusieurs autres auteurs après eux l'ont fait. Mais l'espèce de métaphysique qu'on est obligé d'y employer, est sinon contraire, du moins étrangère à l'esprit de l'analyse, qui ne doit avoir d'autre métaphysique que celle qui consiste dans les premiers principes et dans les opérations fondamentales du calcul.

(Lagrange 1799, p. 233.)

Hence, Lagrange wanted not only to provide a conceptually more convenient basis for the calculus. His principal ambition was to incorporate it within a unitary conception of mathematics based on the "spirit of analysis": a notion we shall try to shed light on. ${ }^{8}$

To do this, Lagrange should have shown that derivative functions could effectively replace differential quotients. Essentially, he should have shown that, for any function $y=f(x)$, there exists an infinity of other functions $f^{(k)}(x)(k=1,2, \ldots)$ that provide, apart from numerical factors, the coefficients of the power-series expansion of $f(x+\xi)$, and formally coincide with the differential quotients $\frac{d^{k} y}{d x^{k}}$. We shall come to this in section 3, bearing in mind Lagrange's notion of function, which we shall deal with in section 2 . For the time being it is only important to remark that both the Théorie and the Leçons begin with an argument that, if correct, would have convinced anybody already familiar with the differential calculus that this condition is met (even if it cannot really be stated, as such, within Lagrange's theory, since it involves the notion of differential quotient, which has no place in this theory). For brevity let us call this argument 'Lagrange's fundamental proof'?

Mathematicians and historians of mathematics have often discussed this argument (the relevant bibliographic references will be given below). Still, in our view, the current accounts, and, more generally, the usual interpretations of Lagrange's theory, do not insist enough on the connections between his technical achievements and his understanding of the crucial notions of quantity, function, and power-series expansion, on which his foundational program is based. These achievements are rather often anachronistically assessed and criticized according to a subsequent understanding of these notions. This tendency has been accentuated by the fact that, during the nineteenth century, several of Lagrange's technical devices and results were isolated from his foundational preoccupations, reinterpreted and used as basic elements of real analysis. For instance, one can mention the notion of transformed function, the remainder theorem, and proof techniques closely resembling the $\varepsilon$ - $\delta$ method (see sections 3 and 5 , and footnote 102).

A comprehensive account of Lagrange's theory in which technical achievements are adequately studied in connection with foundational intents, methodological issues, and philosophical perspectives is therefore still wanting. ${ }^{10}$ The main purpose of our paper is to offer such an account.

When the differences between Lagrange's theory and 19th-century real analysis are considered and the crucial notions of the former are appropriately explained, Lagrange's arguments appear in a new light, and many alleged counter-examples no longer apply. We shall see why this is so. However, in so doing we do not mean to suggest that Lagrange's theory has neither shortcomings nor discrepancies. Many of his arguments are, on the contrary, flawed even when considered in the appropriate context, and are far from constituting unquestionable proofs of the results they are supposed to establish. Furthermore, the actual deployment of the theory is often at odds with the ideal of purity that motivates it. We would like to draw attention to such defects. They help explain why Lagrange's theory was never really accepted by the Continental mathematical community.

${ }^{8}$ If Lagrange's program is so understood, it is not surprising that, after 1797 , he often relied on infinitesimal devices, for example in the second edition of the Mécanique analytique (Lagrange, 1811-1815). Concerning the internal organization of the subject, the Mécanique analytique is indeed quite close to the frame of the Théorie, particularly its mechanical part (see Panza 1991-1992). And for Lagrange this was certainly more relevant, in establishing the place of mechanics and its mathematical methods within the mathematical corpus as a whole, than the local (and non-substantive) use of infinitesimals.

${ }^{9}$ We shall use the term 'proof' and cognates for referring to arguments used, in the context of Lagrange's theory, to establish that something holds, regardless of whether these arguments are actually correct or sound. Analogously, we shall use the term 'theorem' for referring to statements that are taken to hold within this theory, or to re-formulations of these statements that we consider appropriate, regardless of whether these statements actually hold.

${ }^{10}$ Important exceptions are provided by C. Fraser's accounts, especially in Fraser (1987) and (1989). Our views derive, in many respects, from a development of points he makes. 
When he wrote and published the first editions of the Théorie and the Leçons, Lagrange was one of the most influential and respected mathematicians of the large international community to which he belonged, possibly the most influential and respected one. His analytical reformulation of the mechanics of discrete systems, undertaken in the Mécanique analytique (Lagrange 1788), had for example been welcomed, a few years before, as a major achievement, which significantly influenced the development of the subject. The same can be said of the approach to algebraic equations outlined in his treatise De la résolution des équations numériques de tous les degrés (Lagrange 1798), which came out a year after the first edition of the Théorie. The less significant influence his theory of analytical functions had on the Continent ${ }^{11}$ requires an explanation, then.

On the received view, Lagrange's theory is an algebraic treatment of real numbers, both considered as such and as representatives of geometric and mechanical magnitudes. In this picture, the adjective 'algebraic' is intended to evoke two basic features of the theory. On the one hand, it points to the understanding of these numbers as quantities related by operational relations, to the pivotal role assigned to these relations, and to the effort to make the theory as independent as possible both of geometric or mechanical intuition, and of the respective magnitude of the relevant quantities, especially of their being infinitely small or large. On the other hand, it points to the attempt to reduce any real function to polynomial form, so as to limit the use not only of transcendental functions (and numbers), but also of irrational and fractional ones. This double use suggests that Lagrange's theory is algebraic insofar as it carries out a reduction of analysis (both finite and infinitesimal) to algebra, and depicts the latter as an elementary field of study, on which all mathematics should be based, for Lagrange.

On this view, Lagrange's failure to impose his theory is usually explained by observing that such a reduction was manifestly impossible, because of the impossibility of expanding any function in a power series convergent to it. The failure would thus be due to a genuine mathematical mistake: Lagrange's fundamental proof would be flawed insofar as it includes, as a lemma, a wrong theorem, according to which any function has a power series expansion.

This explanation is ineffective. Lagrange'a alleged mathematical mistake is actually a mistake only if his theorem is judged according to alien notions of function and of power series expansion. Hence, if his failure were to be blamed on this theorem, it would depend more on the inappropriateness of the notions he relied on than on his flawed generalizations. To account for this failure, one should then explain, firstly, why Lagrange was working with such inappropriate notions, or rather what made other mathematicians aware of their inappropriateness and pushed them to develop new and more comprehensive notions.

We prefer then a radically different explanation, more intrinsically connected with Lagrange's ideal of purity, and with the general conceptions his theory is based on.

We do not take Lagrange's theory to be about real numbers, neither as such, nor as representatives of geometric and mechanical magnitudes. We rather argue that his theory is founded on a peculiar notion of function which provides quantities that differ fundamentally from both numbers and from geometric and mechanical magnitudes. In our view, numbers and geometric and mechanical magnitudes are, for Lagrange, quantities of a particular sort, whereas his theory of analytical functions is intended to deal with quantities in general, or better in abstracto. We borrow Lagrange's term 'algebraic quantity' to refer to quantities of this sort, and we describe them as relata of a net of operational relations lacking any particular intrinsic feature, characterized only by the sheaf of relations they are involved in (a more precise characterization will of course be offered later). We also insist that, in Lagrange's parlance, the term 'analytical function' —often shortened to 'function' alone-is used with the same meaning, though with a different emphasis. In 'algebraic quantity', the emphasis is on what makes the entities at issue quantities, in a significant sense of this term, that is, on what makes them share some essential features with numbers and geometric and mechanical magnitudes. In 'analytical function', or 'function', the emphasis is on what connects these entities to one another through operational relations displayed by the expressions that denote them, which are of course nothing but the usual expressions entering into mathematical analysis, like ' $x^{2},, \cdot \frac{\sqrt{x}}{a+x},{ }^{\prime} \log x+e^{\frac{a}{x}}$, etc.

It follows that, in our view, Lagrange's theory is not algebraic insofar as it carries out a reduction to algebra understood as a separate elementary field, considered more primitive than this theory. Rather, it is algebraic insofar as it deals with algebraic quantities, that is, insofar as it is not concerned with particular quantities, but with quantities in general. And it is formal, insofar as these quantities are identified through the relations they have with each other, which are in turn displayed by appropriate formulas.

This remains a broad description. It should be clear enough, however, for raising a rather basic problem: what makes algebraic quantities to be quantities in an appropriate significant sense of the term? In other words, what makes the entities denoted by the usual formulas of mathematical analysis to behave the way quantities are supposed to, namely to be ordered linearly, to stay in certain metric relations, and to be subject to certain continuity conditions? In Lagrange's framework (understood as we suggest), this cannot be warranted by independent properties ascribed to the referent of these formulas,

\footnotetext{
${ }^{11}$ For an account of the quite limited "Lagrangian tradition" in the foundation of the calculus, see Grattan-Guinness (1990), vol. 1, sect. 4.3, pp. 195-223. As is well known, Lagrange's theory instead had a notable influence on R. Woodhouse and his followers gathered around the Cambridge Analytical Society, especially C. Babbage, J. Herschel and G. Peacock, who in turn had an important and also well-known influence on the development of 19th-century British mathematics and on the birth of abstract algebra. On the Cambridge Analytical Society, see for instance, Wilkers (1990).
} 
that is, by the features of the intended model on which these formulas are interpreted. For this would amount to reducing algebraic quantities to particular quantities of some sort, thus denying the very raison d'être of Lagrange's theory.

The problem is aggravated by the reductionist ambition of Lagrange's program. Indeed, he purposed not only to foster a particular branch of mathematics, but even to reduce all mathematics to it. But for such a reduction to work, the theory of algebraic quantities had to include general results capable of being interpreted on the particular quantities that mathematics was taken to be about. So Lagrange could hardly avoid requiring that algebraic quantities behave the way particular quantities are supposed to, for otherwise his whole program would have been pointless. Still, reduction should have gone, for him, together with foundation: his aim was to provide a ground for mathematics as a whole, not just a simple framework for a nice reformulation of it. Hence, this requirement had not merely to be imposed on algebraic quantities from the outside, but met because of the very features possessed by algebraic quantities as such.

Our basic point in explaining Lagrange's failure is precisely that his notion of algebraic quantity does not guarantee that algebraic quantities meet this crucial requirement, to the effect that he cannot but surreptitiously suppose that they meet it. Hence, this notion appeared too weak to bear the weight of Lagrange's reductionist purpose. But once reinforced with this surreptitious assumption, it became too strong to play the role of a starting point of his foundational program.

Of course, this basic point has, in our view, different ramifications, capable of accounting for a number of different difficulties to which Lagrange's theory was subject, which made it quite unattractive. We shall come to the details later; what is relevant for the time being is that Lagrange's failure marked, at least on the Continent, the end of the program of eighteenth-century algebraic analysis begun by Euler. By taking the ideal of purity pervading this program to its extreme consequences, Lagrange's theory made it clear that, if conceived as Euler and Lagrange suggested, purity was incompatible with reductionism and foundationalism: if algebraic analysis had to be pure in this sense, the goal of recovering the whole edifice of mathematics within its limits and of grounding this edifice on it could never be reached. This makes the historical interest of this failure and of its explanation clear. Such an interest does not merely rest on the preeminent role Lagrange had in his time and continues to have in the history of mathematics. It is also related to the fact that this failure brings with it the end of a way of doing and conceiving mathematics that characterized a long season of its history. Reacting to Lagrange's foundational perspective and to his ideal of purity was then also a way of promoting a new idea of what mathematics should have been.

This being said concerning the general purpose of our paper, we can describe its plan.

Before undertaking a detailed examination of Lagrange's theory, we consider necessary to clarify his understanding of the crucial notions of quantity, function, and power-series expansion, and, more generally, set out his foundational program and its crucial difficulties. This will be done in Section 2.

In our view, Lagrange's theory includes four main components:

- (i) The fundamental proof;

- (ii) The reformulation of the edifice of the calculus in terms of derivative functions (independently of its geometric and mechanical applications);

- (iii) The remainder theorem;

- (iv) The applications of the algorithm of derivative functions to the solution of particular geometric and mechanical problems.

Section 3 is devoted to component $(i)$.

The other three components are intended to show that the whole corpus of the calculus-including all of its applicationscan be reformulated in terms of derivative functions and their relations, without any appeal to differentials, integrals, or variations. $^{12}$ To carry this purpose out, Lagrange could not limit himself to offer his fundamental proof- so as to guarantee the formal coincidence between the derivative functions $f^{(v)}(x)(v=1,2, \ldots)$ of any function $f(x)$ and the differential quotients $\frac{d^{v} y}{d x v}$ (for $y=f(x)$ ) - and then appeal to an appropriate translation rule for transforming any statement including terms for differentials, integrals or variations in a statement where these terms are replaced by terms for derivative and primitive functions (the primitive function of a given function being, of course, the function having this given function as its derivative). Much of the corpus of the calculus did not, indeed, follow from the algorithm of differential ratios, but rather rested on infinitesimalist arguments, or, more generally, on arguments related to particular interpretations of this algorithm. Thus, once he had provided the fundamental proof, Lagrange still should have shown how to recover anew the totality of this corpus by grounding it only on the place taken by the derivative functions $f^{(v)}(x)$ of any function $f(x)$ in the power series expansions of $f(x+\xi)$.

\footnotetext{
${ }^{12}$ In its first explicit formulation, due to Euler (1744), the calculus of variations involved a special kind of differentials distinguished by usual differentials only for being independent of each other even if the relative variables were functionally related. We call them 'variations'. The reformulation of this calculus as a formalism involving a new operator $\delta$ distinct from $d$ is an important achievement of Lagrange himself, obtained in one of his first scientific memoirs (Lagrange 1761). In the Théorie and the Leçons, he shows then how to reformulate this calculus by relying on nothing but appropriate derivative functions, so as to eliminate the $\delta$-operator itself. On this matter, see Fraser (1985).
} 
Section 4 presents and discusses some examples of component (ii) especially concerned with the reformulation of the theory of differential equations, which Lagrange called 'derivative equations' (Lagrange 1797, arts. 33 and 54; 1801, p. $84 ; 1806$, p. 112; and 1813, arts. I.17 and I.41). This will allow us to illustrate other, more technical difficulties associated with Lagrange's program. We shall show in particular that his actual treatment of derivative equations involves considerable departures from the ideal of purity of method informing this program.

Section 5 deals with the remainder theorem (component (iii)), which is generally considered the major mathematical achievement of the Théorie and the Leçons. To understand how it fits into Lagrange's theory, we begin by considering examples of component (iv) where it applies. We then go through Lagrange's two proofs of this theorem.

Finally, section 6 presents a short conclusion.

The Théorie and Leçons differ in many ways, of which the absence of component (iv) in the Leçons is the most conspicuous. Also the two editions of each treatise respectively differ in several details. But since the theory laid out in the four expositions is essentially the same, a systematic comparative study is unnecessary for our purpose. The annex nonetheless provides information about the different editions and the respective contents of both treatises. It includes four tables: the first shows the place of components $(i)-(i v)$ in these treatises and their respective editions; the three others itemize the different topics included in components (ii) and (iv).

\section{Functions and Algebraic Quantities}

According to a classical way of thinking, dating back to Greek mathematics and philosophy (see Aristotle, Metaphysics, $\Delta, 13,1020 a, 7-14$, and Categories, part 6.), quantities are objects of a specific sort—numbers, for example, or segments or lapses of time-meeting two distinctive requirements: additivity and order comparability. The former specifies that these objects have to be capable of being added up to each other, so as to give rise to other objects of the same sort. The latter specifies that these objects have to be capable of being compared with respect to their size, to the effect that for any two of them, belonging to the same sort, either they are equal, or one of them is greater than the other (and then the latter smaller then the former).

In the Discours préliminaire of the Encyclopédie, d'Alembert makes the following claim:

[...] though, properly speaking, one could only calculate with numbers and the only measurable magnitude is extension (since we could not measure time without space), by generalizing our ideas we reach such a principal part of mathematics and of all natural sciences which is called 'science of magnitudes in general', which is the ground of any discovering that might be made about quantity, that is, about all what is susceptible of increasing or decreasing.

[... ] quoiqu'il n'y ait proprement de calcul possible que par les nombres, ni de grandeur mesurable que l'étendue (car sans l'espace nous ne pourrions mesurer exactement le tems) nous parvenons, en généralisant toûjours nos idées, à cette partie principale des Mathématiques, \& de toutes les Sciences naturelles, qu'on appelle Science des grandeurs en général ; elle est le fondement de toutes les découvertes qu'on peut faire sur la quantité, c'est-à-dire, sur tout ce qui est susceptible d'augmentation ou de diminution.

(Alembert DIS, pp. V-VI.)

This is far from providing a clear and unambiguous definition, but suggests at least the idea that there is a fundamental part of mathematics concerned with quantity conceived in general: not with one or several particular sorts of quantities, but with abstract quantities, so to say ${ }^{13}$. Mutatis mutandis, this is also Lagrange's view. We can try to gain a better understanding of it.

2.1. Lagrange's Definition of Functions. The Théorie begins with an explicit definition of functions:

One calls 'function' of one or several quantities any expression of calculation into which these quantities enter in any way whatsoever combined or not with other quantities which are regarded as having same given and invariable values, whereas the quantities of the function may receive any possible value.

On appelle fonction d'une ou de plusieurs quantités toute expression de calcul dans laquelle ces quantités entrent d'une manière quelconque, mêlées ou non avec d'autres quantités qu'on regarde comme ayant des valeurs données et invariables, tandis que les quantités de la fonction peuvent recevoir toutes les valeurs possibles.

(Lagrange 1797 , art. 1; 1801, p. 6; 1806, p.6; 1813, Introduction, p. 1.) ${ }^{14}$

Lagrange's definition is surprising for us, since, according to it, functions are expressions and contain quantities. This looks incompatible with our familiar distinction between syntactical items and the things some of these items refer to, or, in other words (or more generally), between syntax and semantics. Expressions are syntactical items, indeed, while, in our

\footnotetext{
${ }^{13}$ D'alembert also says (DIS, p. XLIX) that "the object of mathematics is quantity" and repeats that "one calls 'quantity' or 'magnitude' all that can be increased or diminished". This makes clear that he uses 'quantity' and 'magnitude' as synonyms. We shall be more precise and take all magnitudes to be quantities (namely continuous ones), but some quantities (namely discrete ones, such as integer positive numbers) not to be magnitudes. This agrees with Lagrange's use of these terms.

${ }^{14}$ Lagrange often leaves passages largely unchanged, going from the first edition of the Théorie to the Leçons, and from these to the second edition of the Théorie. Where there are some slight changes (as in this case), we quote the most recent version, but include bibliographical references to all the occurrences of the relevant passages.
} 
view, quantities cannot but be things referred to by appropriate expressions, to the effect that an expression might contain terms, some of which might refer to quantities, but it might not contain quantities as such.

One could blame inadvertence, and take 'quantités' in Lagrange's definition to refer not to quantities but to terms referring to quantities, in which case a function would be an expression that includes terms referring to quantities. But there are at least two reasons for discarding such an interpretation.

The first is straightforward: Lagrange's definition resembles many others offered by contemporary mathematicians, ${ }^{15}$ to the effect that inadvertence would then be a quite widespread phenomenon.

The second reason is more complex. Lagrange could have understood functions as expressions including terms referring to quantities only if he had been ready to define quantities without relying on the notion of function. But he was not ready for that, in fact. Against this, one could retort that, following Greek mathematicians, he could have taken quantities to be particular objects defined within appropriate theories, somehow prior to the theory of functions, for example arithmetic, geometry, or mechanics. But if that were the case, there would be arithmetical, geometric, or mechanical functions according to whether they include terms referring to arithmetical, geometric or mechanical quantities. And this openly contradicts the idea that the theory of functions is the most general part of mathematics, which includes all others: an idea that Lagrange doubtless shared with many other 18th-century mathematicians (see: Grabiner 1974, pp. 355-358; Fraser 1987; Fraser 1989; Panza 1992; Panza 1996; Ferraro and Panza 2003).

Lagrange himself suggests an alternative understanding when he remarks, against Newton's theory of fluxions, that:

[...] introducing motion in a calculation whose object is nothing but algebraic quantities is the same as introducing an extraneous idea [...].

[...] introduire le mouvement dans un calcul qui n'a que des quantités algébriques pour objet, c'est y introduire une idée étrangère $[\ldots]$.

(Lagrange 1797, art. 5; 1813, Introduction, p. 3.)

This criticism suggests that the quantities which Lagrange refers to in his definition of functions are algebraic ones. But what are algebraic quantities?

At first glance, the simplest answer would be that algebraic quantities are what algebra deals with. The calculus would then relate to algebraic quantities insofar as it is part of algebra. This was certainly Lagrange's idea, ${ }^{16}$ but it is not enough to appeal to it in order to understand his definition of functions. One should also explain, without appealing to the notion of function, what he took algebra to be. One could consider algebra to be a mere formalism, and algebraic quantities to be what appropriate formulas of this formalism refer to, when they are taken to refer to something. If this were Lagrange's view, his theory would thus be concerned with two sorts of expressions: algebraic formulas and functions. But it is a fact that in Lagrange's treatises there is no trace of such a duplicity.

Rather, Lagrange openly admits that functions are themselves quantities:

The word 'function' has been employed by the first analysts in order to designate in general the powers of a same quantity. Then its meaning has been extended to any quantity however formed by another quantity. Leibniz and the Bernoullis employed it firstly in this general sense, and it is today generally adopted.

Le mot function a été employé par les premiers analystes pour désigner en general les puissances d'une même quantité. Depuis, on a étendu la signification de ce mot à toute quantité formée d'une manière quelconque d'une autre quantité. Leibniz et les Bernoulli l'ont employé les premiers dans cette acception générale, et il est aujourd'hui généralement adopté.

(Lagrange 1797, art. 2; 1813, Introduction, pp. 1-2. See. also: Lagrange 1801, p. 4; 1806, p. 4.)

Lagrange clearly takes his definition to be entirely consistent with this "generally adopted" meaning; namely, he takes it to be consistent with the idea that a function is a "quantity formed in any way from another quantity", as Johann Bernoulli had stated in (1718, p. 241). ${ }^{17}$ In the second edition of the Théorie, he is explicit:

${ }^{15}$ The most notable example is the definition occurring in Euler's Introductio:

A function of a variable quantity is an analytical expression composed in any way whatever of this variable quantity and numbers or constant quantities.

Functio quantitas variabilis est expressio analytica quomodocunque composita ex illa quantitate variabili, et numeris seu quantitatibus constantibus.

(Euler 1748, vol. I, art. 4; here and later, we slightly modify Blanton's English translation: BLA, vol. I, p. 3.)

\footnotetext{
${ }^{16}$ In presenting his course at the École Polytechnique for 1799 (Lagrange 1799, p. 232; see the annex), Lagrange explicitly claims that his aim is to "eliminate the difficulties that one meets in the principles of the differential calculus [...] by linking this calculus immediately to algebra [...]."

${ }^{17}$ Here is Bernoulli's definition:

[...] one calls 'function of a variable quantity' a quantity however composed by this variable quantity and constants. On appelle [...] function d'une grandeur variable, une quantité composée de quelque manière que ce soit de cette grandeur variable et de constantes.

More than fifty years earlier, James Gregory had already advanced a similar definition as being that of quantities:

We say that a quantity is composed by [some] quantities when another quantity results from the addition, subtraction, multiplication, division, root extraction of [these] quantities, or from any other imaginable operation [on them].
} 
Through the character ' $f$ ' or ' $F$ ' placed before a variable, we shall designate in general any function of this variable, that is, any quantity depending on this variable and which varies with it according to a given law.

Nous désignerons en général par la caractéristique $f$ ou $F$, placée devant une variable, toute fonction de cette variable, c'est-à-dire, toute quantité dépendante de cette variable, et qui varie avec elle suivant une loi donnée. (Lagrange 1813, art. I.1.) ${ }^{18}$

It thus seems that for Lagrange a function is a quantity, and that it is so insofar as it is an expression. But then the quantities contained by a function can, according to his definition, in turn be identified with expressions or terms. They are not terms that refer to quantities, but terms that are quantities.

This bring us back to the difficulty raised above, since this understanding is incompatible with our distinction between syntax and the semantics. We nonetheless suggest that Lagrange's notion of function is foreign to such a distinction and can only be understood if this distinction is dispensed with.

To see how, consider two further quotations, from the Leçons and a short paper Lagrange published in 1799 in the Journal de l'École Polytechnique:

[...] one should regard algebra as the science of functions, and it is easy to see that the solution of equations does not consist in general but in finding the values of unknown quantities as determined functions of known quantities. These functions represent, then, the different operations that have to be performed to the known quantities in order to get the values of those which are sought, and they are properly only the last result of the calculation. But in algebra, one considers functions only insofar as they result from the operations of arithmetic, these operations having been generalized and transferred to letters, whereas in the calculus of functions strictu sensu, one considers functions that result from the algebraic operation of expansion in series when one assigns indeterminate increments to one or several quantities of the function.

[...] on doit regarder l'Algèbre comme la science des fonctions; et il est aisé de voir que la résolution des équations ne consiste, en général, qu'à trouver les valeurs des quantités inconnues en fonctions déterminées des quantités connues. Ces fonctions représentent alors les différentes opérations qu'il faut faire sur les quantités connues pour obtenir les valeurs de celles que l'on cherche, et elles ne sont proprement que le dernier résultat du calcul. Mais, en Algèbre, on ne considère les fonctions qu'autant qu'elles résultent des opérations de l'Arithmétique, généralisées et transposées aux lettres, au lieu que dans le Calcul des fonctions proprement dit, on considère les fonctions qui résultent de l'opération algébrique du développement en série lorsqu'on attribue à une ou à plusieurs quantités de la fonction, des accroissements indéterminés.

(Lagrange 1801, p. 4; 1806, p. 4.) $)^{19}$

Strictly speaking, algebra in general is nothing but the theory of functions. In Arithmetic, one looks for numbers according to given conditions between these numbers and other numbers; and the numbers that are found meet these conditions without conserving any trace of the operations that were needed in order to form them. In algebra, instead, the sought after quantities have to be functions of given quantities, that is, expressions representing the different operations that have to be performed on these quantities in order to get the values of the sought after quantities. In algebra stricto sensu, one only considers primitive functions that result from ordinary algebraic operations; this is the first branch of the theory of functions. In the second branch, one considers derivative functions, and it is this branch that we simply designate with the name 'theory of analytical functions', and that encompasses all that which pertains to the new calculi.

À proprement parler, l'Algèbre n'est en général que la théorie des fonctions. Dans l'Arithmétique, on cherche des nombres par des conditions données entre ces nombres et d'autres nombres; et les nombres qu'on trouve satisfont à ces conditions sans conserver aucune trace des opérations qui ont servi à les former. Dans l'Algèbre, au contraire, les quantités qu'on cherche doivent être des fonctions des quantités données, c'est-à-dire, des expressions qui représentent les différentes opérations qu'il faut faire sur ces quantités pour obtenir les valeurs

Quamitatem dicimus à quantitatibus esse compositam; cum à quantitatum additione, subductione, multiplicatione, divisione, radicum extractione, vel quacunque alia imaginabili operatione, fit alia quantitas.

(Gregory 1667, p. 9; see Youschkevitch 1976-1977, p. 58.)

According to Gregory, quantities so defined are analytically composed just in case that the relevant operations are algebraic (in modern sense):

When a quantity is composed by the addition, subtraction, multiplication, division, [or] root extraction of [some] quantities, we say that it is analytically composed.

Quandò quantitas componitur ex quantitatum additione, subductione, multiplicationr divisione, radicum extractione; dicimus illam componi analyticè.

(Gregory 1667, p. 9.)

This use of the adjective 'analytical' and its cognates is thus more restrictive than Lagrange's: see 2.3 section below.

${ }^{18}$ Lagrange does not enclose the argument of a function of one variable in parentheses when the argument is expressed by an atomic symbol: he writes ' $f x$ ' when we would rather write ' $f(x)$ ', but he writes ' $f\left(x^{2}\right)$ ', ' $f(x+\xi)$ ' and ' $f(x, y)$ ' as we do. He furthermore writes ' $f$ ' $(x)$ ' for the derivative of a function of several variables with respect to $x$. For simplicity we adhere to modern notational conventions even in our quotations from Lagrange.

${ }^{19}$ This passage is quoted by J. L. Ovaert (Ovaert 1976, p. 172) to emphasize the "algebraic character" of Lagrange's notion of function. 
des quantités cherchées. Dans l'Algèbre proprement dite, on ne considère que les fonctions primitives qui résultent des opérations algébriques ordinaires; c'est la première branche de la théorie des fonctions. Dans la seconde branche on considère les fonctions dérivées, et c'est cette branche que nous désignons simplement par le nom de Théorie des fonctions analytiques, et qui comprend tout ce qui a rapport aux nouveaux calculs. (Lagrange 1799, p. 235.) ${ }^{20}$

These two quotations suggest that 'algebra' and 'theory of (analytical) functions' (that we take to be synonymous of 'science of functions' and 'calculus of functions') are, for Lagrange, two names for the same theory-also known as 'analysis' or better 'algebraic analysis', after the complete title of the Théorie (see p. 1, above)—, or at any rate, two names of two intimately connected branches of this very theory.

This theory concerns quantities that, in Lagrange parlance, are viewed as "expressions that represent operations" that are to be performed for passing from one quantity to another. ${ }^{21}$ More precisely, it deals with the system of relations induced by the indefinite composition of certain elementary operations applied to indeterminate arguments. ${ }^{22}$ Though these $^{2}$ arguments are indeterminate as such, they can be identified by looking at the net of relations in which they occur. Once this is done, they become quantities, namely algebraic quantities. Hence, quantities and expressions are not distinct for Lagrange because neither operations nor their arguments are there before the corresponding symbols. First there are only symbols and the formulas they make up, which are subject to rules of composition and transformation. Operations and quantities appear next, whenever these symbols and formulas are supposed to express something. Thus, for Lagrange, expressions do not refer to operations or quantities that are there independently of them; they constitute, or rather generate, operations and quantities. The universe of his theory of functions is a universe of symbols governed by rules of composition and transformation, not a universe of objects and relations which these symbols refer to.

In Lagrange parlance, the verb 'to express' should therefore not be taken to mean the same as 'to refer to': an expression does not express a quantity insofar as it refers to it, but rather insofar as it can be taken to be this very quantity, or, as Lagrange admits without any reluctance, insofar as it is this quantity. In the same vein, the verb 'to represent' should not be taken to evoke a relation linking two items that might be given independently of each other: an expression represents an operation insofar as it expresses (or better is) a quantity which is viewed as the result of performing this operation on another quantity. It follows that, for Lagrange, a quantity is algebraic insofar as it is a quantity whose identity merely depends on its being expressed by an appropriate expression, and is then to be conceived as nothing but a relatum of the net of relations corresponding to the operations that this very expression represents. ${ }^{23}$

To take a simple example, insofar $x$ is not taken to be a quantity of a certain particular sort, $x^{2}$ is an algebraic quantity which stays in the two-places relation $\ulcorner$ (to be the) square of $\urcorner$ with the other algebraic quantity $x$. It is then characterized as such (that is, as this very quantity rather than any other one) merely by its being expressed by the expression ' $x^{2}$, , and is consequently to be conceived as nothing but a relatum of this two places relation (which links it to the other relatum $x$, and corresponds to the operation of square power). Hence, the expression ' $x^{2}$ ' makes different things at once: it expresses the algebraic quantity $x^{2}$, in the sense that it is this very quantity; it expresses the relation that links this quantity to $x$; it furthermore represents the operation of square power insofar as it is applied to $x$ and gets $x^{2}$.

Once this is accepted, Lagrange's definition of functions becomes clear. Insofar as expressions are quantities, for him, and quantities are to be taken, in the context of (the pure part of) his theory of analytical function, as algebraic (rather than as particular) ones, functions are twofold entities: they are expressions insofar as they express (algebraic) quantities, and (algebraic) quantities insofar as they are expressed by appropriate expressions. The following definition, which Lagrange advance in his treatise on numerical equations, is fairly explicit:

[...] when a quantity depends on other quantities in such a way that it can be expressed by a formula that includes these quantities, one says then that it is a function of these same quantities.

[...] lorsqu'une quantité dépend d'autres quantités, de manière qu'elle peut être exprimée par une formule qui contient ces quantités, on dit alors qu'elle est une fonction de ces mêmes quantités.

(Lagrange 1798, p. VII; 1808, pp. 14-15.) ${ }^{24}$

\footnotetext{
${ }^{20} \mathrm{~A}$ similar claim is also made in manuscript 1323 of the library of l'École des Ponts et Chaussées (see the annex): "[...] le calcul des fonctions [... ] n'a donc rien qui le distingue de l'algèbre proprement dite." (Pepe 1986, p. 31.)

${ }^{21}$ The idea that an expression "represents operations " seems already implied in Lagrange's use of the term 'expression of calculation', as opposed to the term 'expression' tout court, in his definition of functions.

${ }^{22}$ According to Grabiner (1990, p. 72): "if Lagrange said he would reduce the calculus to algebra, he meant that its subject matter would be systems of operations which are expressible by symbolic formulas.” See also Fraser (1987), p. 39.

${ }^{23}$ This way of thinking quantities was in no way specific to Lagrange, though Lagrange was certainly the mathematician that tried, more than any other one, to reform the calculus in conformity with this conception. A crystal clear example is offered by Klügel (1800, p. 146). Rather than expressions or functions, he speaks of forms, but his basic idea is just the same as Lagrange's: in mathematics, the form is the "modality of composition of a quantity by another quantity [Art der Zusammensetzung einer Größe aus andern Größen]", and mathematics as a whole is "the science of the form of quantities [Wissenschaft der Formen der Grossen]".

${ }^{24}$ This quotation should be enough to show that the opposition proposed by Youschkevitch (1976-1977) and Monna (1972) between two notions of function in 18th-century mathematics - according to which a function is respectively an expression or a quantity depending on other quantities-is groundless. On this matter, see: Panza (1992), ch.II.2, especially sect. II.2. $\eta$; Panza (1996); Ferraro (2000).
} 
All these considerations should be enough in order to clarify the first part of Lagrange's definition of functions, according to which a "function of one or several quantities" is an "expression of calculation into which these quantities enter in any way whatsoever combined or not with other quantities". But more is needed for understanding what does it mean that these last quantities "are regarded as having same given and invariable values, whereas the quantities of the function may receive any possible value", and, more generally, for explaining how functions enter into Lagrange's theory. The following subsections 2.2-2.7 are devoted to this.

2.2. Functions and Numbers. We begin by discussing a possible objection to the outlined understanding of Lagrange's definition.

At the beginning of the Introductio, Euler claims that "numbers of any sort" are constant quantities, and that- "since all determined values" that a variable quantity "encompasses within itself" can be expressed by numbers-“a variable quantity involves all numbers of any sort" (Euler 1748, vol. I, arts. 1-2; Euler BLA, vol. I, p. 2). One could take these claims - together with Euler's practice of assigning numerical (real or complex) values to variable quantities-to suggest that quantities are just numbers for Euler. One could then argue that the same applies to Lagrange. ${ }^{25}$ Though we do not in fact feel that Euler identified quantities with numbers, we shall stick to Lagrange (concerning Euler, see Panza 2007, sect. 1.1).

In the two passages from the Leçons and the 1799 paper of the Journal de l'École Polytechnique quoted above (pp. 7 and 8), Lagrange explicitly distinguishes algebra or theory of functions from arithmetic, and explicitly uses the terms 'quantity' and 'number' to speak of the entities they deal with, respectively. This explicitly suggests what is also implicitly suggested by Lagrange's theory as a whole: that numbers are for him quantities of a particular sort, whereas the theory of functions deals with quantities in general, i. e. with algebraic quantities.

The presence of numerals in many formulas occurring in Lagrange's treatises does not constitute evidence to the contrary. Numerals admit, indeed, an easy interpretation as symbols either for constant quantities characterized by a particular operational role or for indices of operations.

To begin with, symbols like ' 0 ', ' 1 ', can be easily understood as symbols for constant quantities characterized by a particular operational role. Concerning ' 0 ' a similar understanding is natural: ' $x+y=0$ ' is, for example, shorthand for ' $x=-y$ '. Concerning ' 1 ', a similar understanding is explicitly fixed by Descartes in his Géometrie, where he takes this symbol to refer to the unity of segments conceived as the neuter element for multiplication on them (Descartes 1637 , pp. 297-300; Panza 2005, pp. 23-27). There is no reason to think that Lagrange does not accept and generalize Descartes' stipulation on this matter, so as to take ' 1 ' to express (in the sense clarified above) the neuter element of multiplication on abstract quantities.

If this is accepted, any numeral usually understood as referring to an algebraic number (in modern sense) can be conceived as a symbol either expressing a quantity resulting from appropriate operations applied to such a neuter element, or indicating such operations themselves. To take the simplest example, ' 2 ' in ' $x+2$ ' can be viewed as shorthand for ' $1+1$ '.

A similar understanding also applies to symbols like ' $e$ ' or ' $\pi$ ' in expressions like ' $e$ ' or ' $\frac{\pi}{2}$ '. They can also be taken as symbols for constant quantities characterized by a certain operational role to be specified by defining the logarithmic, exponential, and trigonometric functions.

On the other hand, expressions like ' $x^{3}$ ' or $\sqrt[3]{2}$ ' or display the use of numerals (the numeral ' 3 ', in these cases) as symbols for indices of operations. Under this use, numerals clearly refer to numbers, but these numbers are not the quantities which Lagrange's theory is is dealing with, but are rather there to help in designing certain operations on these last quantities. Thus, ' $x^{3}$ ' is to be read as the expression of the result that is got by multiplying the quantity $x$ three times by itself, whereas $\sqrt[3]{2}$ ' is to be read as the expression of the quantity that, if multiplied three times by itself, yields the quantity 2 (or $1+1$ ). A similar understanding also applies to ' $3 x$ ' or ' $\frac{3}{x}$, , which are then to be viewed as shorthand for ' $x+x+x$ ' and ' $\frac{1}{x}+\frac{1}{x}+\frac{1}{x}$ ', respectively.

2.3. Why Lagrange's Functions are Analytical? Another relevant question concerns Lagrange's use of the adjective 'analytical' in the term 'analytical functions'.

In Lagrange's treatises, this adjective is clearly not intended to qualify a particular class of functions. Lagrange may have borrowed the term 'analytical functions' from Condorcet's unpublished Traité du calcul integral. ${ }^{26}$ But he uses it differently, namely to refer to the totality of functions, understood as we have suggested. This is also Dugac's view (2003, p. 71), according to which the adjective 'analytical', in the title of the Théorie, has the sense given to it by d'Alembert in the article "Analytique" of the Encyclopédie: according to this sense, it merely applies to all that "belongs to the analysis, or is of the same nature as analysis, or is done through analysis" (Alembert ANA, p. 403).

\footnotetext{
${ }^{25}$ We thank Jesper Lützen for having attracted our attention on this point.

${ }^{26}$ The manuscript of Condorcet's treatise is conserved at the Library of the Institut, in Paris. On the occurrence and meaning of the term 'functions analytiques' in such a manuscript, see Youschkevitch (1976-1977), pp. 75-76, and Gilain (1988), p 103.
} 
2.4. In what Sense are Functions Quantities? After these two preliminary questions (those addressed in sections 2.2 and 2.3, respectively), we move to a more substantial matter. Our understanding of Lagrange's definition of functions leaves a problem open: in what sense can algebraic quantities be considered quantities? Or rather, what do they have in common with quantities of a particular sort, such as numbers, segments or speeds, that allows one to view them as quantities too?

One could think that what is crucial here is that algebraic quantities are arguments of operations supposed to have the same formal properties as the usual operations on numbers or segments. This is only a partial answer, however.

Indeed Lagrange assigns to algebraic quantities certain features that do not merely depend on their being expressed by appropriate expressions. He attributes to them, namely, a linear order and certain metric relations, and assumes they respect certain conditions of continuity. In a word, he assumes that algebraic quantities, even if they are not numbers, behave in many respects the way real numbers, or better, oriented segments (a null segment included) do. For short, we can term 'real' those quantities that behave this way. It follows that, for Lagrange, algebraic quantities are real ${ }^{27}$.

This assumption has a crucial consequence: it implicitly introduces a discrepancy between the understanding of functions as expressions and their understanding as quantities, and this gives rise to an important discrepancy between Lagrange's ideal of purity and the actual deployment of his theory. On the one hand, Lagrange's ideal of purity requires functions to be studied merely as expressions; on the other, many arguments in his theory rely on properties that functions have insofar as they are real quantities. ${ }^{28}$

It is a matter of fact that Lagrange takes algebraic quantities to be real. But—one could wonder-why does he do it? The simple remark that this supposition occurs in some of his crucial arguments is not enough to answer the question. The crucial point is rather that Lagrange uses these arguments because the very purpose of his theory is that of reducing the study of any particular sort of quantities to the study of functions. Indeed, to do this, he must do more than just providing a theory of functions. He has to show, or at least to suggest, that all the known results concerned with particular sorts of quantities may be recovered within this theory. This is precisely what forces him to suppose that algebraic quantities are real.

The double concern of Lagrange's theory for algebraic quantities and for particular sorts of quantities is reflected by a separation that is made manifest by the table of contents of the Théorie. This is the separation of this theory into two distinct parts: a pure part dealing with functions in general, including components (i)-(iii) among the four ones distinguished at p. 4; and an applicative part dealing with particular sorts of quantities, including element (iv). Now, on one hand, this separation is downplayed by the fact that the first part is greatly influenced by the aim of making geometric and mechanical applications possible: these applications do not appear as mere corollaries of the fundamental principles of the theory; they are rather built into these same principles, especially into the remainder theorem. ${ }^{29}$ On the other hand, this same separation is not only reflected by the very structure of Lagrange's treatises. It is also emphasized by Lagrange's effort of keeping formal considerations as detached as possible from considerations concerning order, metric

\footnotetext{
${ }^{27}$ At first glance, this claim appears to contradict the argument Lagrange relies on to exclude the possibility that "in the series that results from the development of a function $f(x+\xi)$ there cannot be any fractional powers of $x$ " (Lagrange 1797, art. 10; 1801, p. 8; 1806, p. 9; 1813, art. I.2; see section 3.1, below). As we shall show in section 3.1.3, below, this argument can be justified, however, without denying that algebraic quantities are real. In another case-namely in his treatment of trigonometric functions in both editions of the Théorie (Lagrange 1797, arts. 25-29; 1813, arts. I.14 and I.18-32)—Lagrange relies on imaginary coefficients and exponents (explicitly identified as such through the occurrence of the factor $\sqrt{-1}$ ), but these are handled in a purely formal way and are used to study the sine and the cosine functions under the implicit assumption that their arguments are real quantities.

${ }^{28}$ This discrepancy should not conceal that Lagrange's ideal of purity forces him to treat algebraic quantities as far as possible as pure relata of the net of relations expressed by the relevant expressions. This attitude was famously contrasted by many coeval mathematicians. When this criticism is mentioned, Cauchy's rejection of the "arguments drawn from the generality of algebra", made clear in the preface of his Cours d'analyse (Cauchy 1821 , p. ii; CABS, p. 1), is often quoted. A much earlier example of this criticism is the following passage included in a memoir by Ampère presented to the Institut des sciences in 1803 and appeared in 1806:
}

That which is termed a fact of analysis has always to be reduced to the metaphysical principles of this science, if one wants to have a right idea of it. It is evident, indeed, that one has always to find the reasons of all results obtained through calculation in the attentive examination of the conditions of any question, since the use of algebraic characters can adds nothing to the ideas that they represent.

Ce qu'on appelle un fait d'analyse doit toujours être ramené, si l'on veut s'en faire une idée juste, aux principes métaphysiques de cette science. Il est évident, en effet, que l'emploi des caractères algébriques ne pouvant rien ajouter aux idées qu'ils représentent, on doit toujours trouver dans l'examen attentif des conditions de chaque question la raison de tous les résultats où l'on est conduit par le calcul. (Ampère 1806, p. 496.)

\footnotetext{
${ }^{29}$ This is to say that Lagrange's theory satisfies, with respect to its geometric and mechanical applications, what today's philosophers of mathematics usually call 'Frege's constraint': see Dummett (1991), p. 274; Wright (2000), p. 324.
} 
and continuity, ${ }^{30}$ of developing the pure part of the theory as far as possible without appealing (at least explicitly) to order, metric and continuity for algebraic quantities.

In this context, the explicit recourse to conditions of order and continuity that appears in the proofs of the remainder theorem is striking, since it appears as an inevitable deviation from a basic methodological purpose. The same holds for other surreptitious recourses to these conditions in other crucial arguments included in the pure part of Lagrange's theory, even in some quite crucial ones, as the fundamental proof itself.

The idea that the study of quantities of any particular sort has to be reduced to the study of functions has another important consequence. As we have said above, algebra for Lagrange coincides with the theory of (analytical) functions or is at least intimately connected with it, insofar as the latter as well as the former are branches of the same more general theory. As the study of quantities is just mathematics, it follows that this more general theory is not a branch of mathematics, but a unitary framework to which all mathematics is to be reduced. Hence, speaking of algebra or of the theory of (analytical) functions is not for Lagrange a way of speaking of a portion of mathematics, but rather a way of speaking of mathematics as a whole, appropriately understood. In fact this use of the term 'algebra' is not surprising: on the one hand, the conception of algebra as a well delimited domain within mathematics is quite modern and in the eighteenth century it was far from being generally accepted; on the other, the possibility of expanding any function in a power series-a possibility that, as we shall see in section 3, Lagrange believed he had established-strongly suggests that any function can be recast in the form of a polynomial expression.

2.5. Variables, Constants and Indeterminate Quantities. We are now ready for considering Lagrange's distinction between the quantities that "are regarded as having same given and invariable values", and those that "may take on any possible value", i. e. the distinction between constant and variable quantities.

There are two points to be clarified in connection with this distinction: how should we understand, in the context of Lagrange's theory, the notion of a value of a quantity? What does it mean that a quantity may take on not just several values, but rather all possible values?

Let us begin with the first point.

Lagrange's theory appears, to a modern mathematician, as a (flawed) version of real analysis. From this point of view, it is natural to admit that Lagrange's variables vary over the real numbers and his constants are real numbers. As said, we do not share this understanding (though we recognize, of course, that variables and constants can, for Lagrange, be interpreted as numbers). So we have to suggest an alternative understanding of Lagrange's notion of value.

We suggest that, for Lagrange, a value of an algebraic quantity is another algebraic quantity entitled to replace the former, and that may be explicitly associated with it by means of an appropriate equality. A constant algebraic quantity is thus an algebraic quantity that—within a certain argumentative context—does not admit arbitrary replacements, that is, either does not admit replacements at all, or admits only some suitable replacements. A variable algebraic quantity is instead an algebraic quantity that—again, within a certain argumentative context—admits any arbitrary replacement compatible with the rules of composition. So conceived, the distinction between constant and variable algebraic quantities not only depends on the formal treatment of these quantities, it is also relative to argumentative contexts.

According to Lagrange, some algebraic quantities can also be taken to be indeterminate. This means that, within a certain argumentative context, they are handled independently of their having, or their being able to take, a certain value and then, of their admitting or not some replacements. Often, indeterminate quantities are supposed to be whichever constant quantities, and are then open to take whichever suitable value, and even to be later considered as variable. This is clearly the case of the increment $\xi$ in $f(x+\xi)$.

Let us consider now the second point.

We have already mentioned what Euler says about quantities and numbers at the beginning of the Introductio. Besides this, he also claims that a variable quantity "encompasses within itself, in general, any determined value", and that "a variable quantity is the genus in which are contained all determined quantities" (Euler 1748, vol. I, art. 2; BLA, vol. I, pp. 2-3). Moreover, he adds that a "function of a variable" is a variable quantity, in turn, which means that it takes on any determined value. More precisely, he argues that, "since it is permitted to substitute all determined values for the variable quantity, the function takes on innumerable determined values", and adds that "no determined value is excluded from those which the function can take on, if the variable quantity also involve imaginary values" (Euler 1748, vol. I, art. 5; BLA, vol. I, p. 3; on Euler views on this matter, we refer the reader to Panza 2007, sect.1.1).

Lagrange merely repeats the first of Euler's claims, but he does not try to explain it, and does not insist on its consequences relative to the values that a function may take on, in turn. On the top of that, in expounding his theory, he even seems to make at least two concessions that contradict Euler's second claim. On the one hand, he seems to admit the possibility of taking a constant as a function of any variable. On the other, he seems to grant that a function might

\footnotetext{
${ }^{30}$ Ovaert (1976, p. 173) has argued that in the Leçons, though not in the Théorie, Lagrange is striving to sever the "formal point of view" from the "numerical point of view". On this point, see also Alvarez-Jimenez (1997), pp. 121 and 125, according to which Lagrange's theory presents "two levels", the first being given by a "purely formal representation for functions", and the second by the "effective calculation" of "numerical value of a function". The adjective 'numerical' seems to us inappropriate, since quantities of a particular sorts do not have do be numbers. Still, these remarks seem to us to capture an important feature of Lagrange's theory.
} 
be such that its variable be not licensed to take on any value. The first concession occurs quite locally in Lagrange's theory, for example in his treatment of singular solutions of derivative equations (we shall return to this matter in section 4.2), though it is implied by the acknowledgment (that is natural in view of his notion of function) that expressions like ' $2 a^{2}-a x-a(a-x)$ '-in which a variable occurs, as it were, only vacuously - are or express functions. The second concession pervades the whole theory, instead, and it is much more relevant.

To see why, consider two simple examples, namely the expressions: ' $\frac{a}{a-x}$ ' and ' $\sqrt{a-x}$ '. It seems that, in Lagrange's view, expressions like these are not well-formed if $x$ takes some particular values: for ' $\frac{a}{a-x}$ ', this is the case if $x$ takes the value $a$; and for ' $\sqrt{a-x}$, this is the case if $x$ takes any value greater than $a$.

These two examples are very different. To argue that ' $\frac{a}{a-x}$ ' is not well-formed if $x=a$ it is enough to maintain that division by 0 (i.e. by the neuter element of addition on abstract quantities) is not allowed, or that ' $\frac{a}{0}$ ' is meaningless since there is no such thing as an infinite quantity. To argue that the expression ' $\sqrt{a-x}$ ' is not well-formed if $x>a$, one has to say that no quadratic form is negative, or that ' $\sqrt{a-b}$, is meaningless if $b>a$, since there is no quantity whose square is negative. Lagrange seems to implicitly endorse both these arguments. In a sense, this is natural, since they perfectly fit with the assumption that algebraic quantities are real. But, this has also strong consequences on the pure part of the theory.

According to our modern set-theoretic understanding, a function is a mapping $f: X \rightarrow Y$ from a certain set $X$ to a certain set $Y$, and its very nature depends not only on the rules the mapping is complying with, but also on the nature of these sets. For Lagrange, a function is, instead, an expression that expresses a quantity. There is thus no way to specify beforehand a certain domain of values of its variable (or variables) where it is defined. Still, once it is assumed that algebraic quantities are real, and that functions express them, it becomes necessary to assign to functions something like a domain of definition.

We employ this last term for short, but we insist on the fact that what we use it to refer to is quite different from what this last term refers to in a set-theoretic context. In the context of Lagrange's theory, the domain of definition of a function is given by the possible substitutions for its variable (or variables) that preserve its property of expressing a real quantity, thus allowing the function to subsist as such. So understood, the domain of definition of a function is not established independently of the expression that constitutes the function itself, but is imposed by it.

It follows that for Lagrange, insofar the functions $\frac{a}{a-x}$ and $\sqrt{a-x}$ are not defined for $x=a$ and $x>a$, respectively, they have simply no property under the condition that $x=a$ and $x>a$. To claim, for example, that the first of them is discontinuous for $x=a$ is simply nonsensical: this function is neither a mapping from $\mathbb{R}$ to $\mathbb{R}$, nor a mapping from any set $X$ including the value $a$ to any other appropriate set $Y$; it is just the expression itself insofar as it expresses a real quantity; and this last condition is precisely what does not obtain if $x=a$.

Strictly speaking, such a conception contradicts Lagrange's claim that a variable quantity "may take on all possible values", at least if it is admitted that functions like $\frac{a}{a-x}$ and $\sqrt{a-x}$ are variable quantities, which Lagrange could have not denied. But there is a sense in which this conception is entirely consistent with this claim. According to the former, the values that a variable occurring in a function can take on cannot be established at will. It follows that Lagrange's functions are not only defined everywhere on their domain of definition, but also that in Lagrange's theory there is no room for piece-wise defined functions like the following:

$$
f(x)=\left\{\begin{array}{cc}
x^{2} & \text { for } x \geq 0 \\
x & \text { for } x<0
\end{array},\right.
$$

as already observed by Fraser (1987, pp. 40-41).

2.6. The Compositional Conception of Functions. It is now time to consider a question we have so far left aside: what sort of expression is a function?

Lagrange adds to his definition neither a list of admitted atomic symbols that can compose functions, nor a specification of the rules of composition of these symbols. He merely takes it for granted that the reader is familiar with the formalism used to provide the relevant expressions, and there is thus no need of fixing it.

The crucial point here is however not concerned with the choice and delimitation of this formalism, but rather with the compositional conception of functions that Lagrange seems also to take for granted. His endorsement of this conception is made clear by the following claim, which he makes after having established the power-series expansions of $(x+\xi)^{m}$, $a^{(x+\xi)}, \log _{a}(x+\xi), \sin (x+\xi)$ and $\cos (x+\xi)$ :

The functions $x^{m}, a^{x}, l x{ }^{31} \sin x, \cos x$ we have just considered can be regarded as the simple analytical functions of only one variable. All other functions of the same variable are either composed by these ones through addition, subtraction, multiplication, or division, or are given in general through some equations into which some functions of this same forms enter.

Les fonctions $x^{m}, a^{x}, l x, \sin x, \cos x$ que nous venons de considérer, peuvent être regardées comme les fonctions simples analytiques d'une seule variable. Toutes les autres fonctions de la même variable se composent de

\footnotetext{
${ }^{31}$ In Lagrange's notation, $l x$ is the Neperian logarithm of $x$.
} 
celles-là par addition, soustraction, multiplication ou division; ou sont données en général par des équations dans lesquelles entrent des fonctions de ces mêmes formes.

(Lagrange 1797, art. 30; 1813, art. I.15; see also Lagrange 1801, p. 36; 1806, p. 47.)

Like Euler (1748, vol. I, ch. VIII), Lagrange seems to include both the sine and the cosine among simple functions, but no inverse trigonometric function. ${ }^{32} \mathrm{He}$ also neglects to observe that functions can compose by replacing their arguments with other functions, though, in his treatises, he makes a great use of such a form of composition, of course.

Leaving aside these harmless flaws, his point is clear: functions are generated by finitary algebraic compositions of a handful of elementary (or simple) ones ${ }^{33}$ or are implicitly defined through equations involving functions generated in this way. This point deserves further clarification.

Lagrange's acceptation of exponential, logarithmic and trigonometric functions is not at odd with his understanding of functions as algebraic quantities. Since, as we have seen above, for him algebra is not a portion of mathematics, but mathematics as a whole, appropriately conceived. In the context of Lagrange's (extended) algebra, trigonometric functions do, however, have a different role from exponential and logarithmic functions. The functions $a^{x}$ and $\log _{a} x \operatorname{can}$ be viewed as resulting from a generalization of elementary arithmetical operations, and their presence among elementary functions is thus perfectly consistent with the "genetic" (Vuillemin 1962, p. 64; Gusdorf 1971, pp. 232-249) conception that was shared by many coeval mathematicians, according to which mathematics is gradually generated by extension, starting from an elementary base. ${ }^{34}$ This is not so for trigonometric functions, whose basic properties depend, rather, on their geometric origins. It is true that the sine and the cosine can also be defined through imaginary exponentials (see footnote 27, above). But if they are so defined, they are no longer elementary function, and there is no reason for assigning to them a relevant role in the theory of functions. The fact that Lagrange's elementary functions include the functions sine and cosine is thus another case of discrepancy between Lagrange's ideal of purity and the actual deployment of his theory guided by its reductionist purpose.

Another relevant question is concerned with the last part of the claim quoted above, where Lagrange says that functions can be "given" through equations. Later, Lagrange confirms this claim by remarking that "the function $y$ could be given through any equation between $x$ and $y$ " (Lagrange 1797, art. 33; 1801, p. 47; 1806, p. 52; 1813, art. I.17). Right after this second claim, Lagrange proves that if $F(x, y)=0$, then the first derivative of $y$ is $-\frac{F^{\prime} x(x, y)}{F^{\prime} y(x, y)}$, where $F^{\prime} x(x, y)$ and $F^{\prime} y(x, y)$ are the first derivatives of $F(x, y)$ taken respectively with respect to $x$ and to $y$ (see equality (21, below).

This suggests that here Lagrange is referring only to non-derivative equations. He therefore does not seem to countenance the possibility that a function can be implicitly defined through a derivative equation. What he appears to have in mind is that functions are either elementary or derive from a finitary algebraic composition of elementary functions, or from the solution of an equation involving elementary functions of two variables, algebraically composed. For short, call this the 'compositional conception' (of functions).

The trouble with this conception is that it is at odds with a number of arguments and results occurring in the Théorie and the Leçons. We shall later consider two of them (see sections 3 and 4.4, respectively): the first is Lagrange's fundamental proof, no less; the second concerns partial derivative equations.

2.7. Generality. The last point we want to make about Lagrange's notion of function concerns its relation with a typical feature of Lagrange's proofs, namely his efforts to avoid as far as possible the assignment of constant values to some variables (Ovaert 1976, pp. 173; Fraser 1987, pp. 44).

This attitude is quite natural if functions are conceived as expressions. For a function so conceived can dramatically alter its nature when a symbol that occurs in it is replaced with another. Consider the function $x^{2}+\sqrt{x-a}$. If the variable $x$ takes the value $a$, the radical disappears and the function loses one of its essential features: that of including a fractional power. Hence, Lagrange merely wants to avoid the mistakes that could derive from such an alteration, if it happens surreptitiously.

\footnotetext{
${ }^{32}$ But note that in the Leçons (Lagrange 1801, pp. 35-36: 1806, pp. 45-46), he obtains the derivatives of arcsin $x$ and arccos $x$ by inverting the power-series expansion of $\sin (x+\xi)$ and $\cos (x+\xi)$.

${ }^{33}$ Here and below we use the term 'composition' and its cognates in relation with functions in a sense broader than that in which this term is habitually used today. In this broad sense, two functions $f(x)$ and $g(x)$ can be composed by going over not only the functions $f(g(x))$ and $g(f(x))$, but also the functions $f(x)+g(x), f(x)-g(x),[f(x)][g(x)]$, and $\frac{f(x)}{g(x)}$, that is, as Lagrange says, by addition, subtraction, multiplication or division.

${ }^{34}$ This conception was especially promoted by Condillac in his influential essay La langue des calculs (Condillac 1797). For a study of this essay, see Dhombres (1982-1983). The following passage drawn from the Leçons provides evidence for Lagrange's acceptance of this conception in the case of the exponential function:

The function $x^{m}$, in which $x$ is the variable and $m$ is a constant, naturally brings to the consideration of the function $a^{x}$, in which the variable is $x$, and $a$ is a constant.

La fonction $x^{m}$, dans laquelle $x$ est la variable et $m$ est une constante, conduit naturellement à la considération de la fonction $a^{x}$, dans laquelle la variable est $x$, et où $a$ est une constante.

(Lagrange 1801, p. 20; 1806, p. 25.)
} 
But that is not all. What is more relevant is that the very possibility of such an alteration of the nature of a function goes together with a conception of generality concerning functions that is essentially different from the one we are familiar with. Lagrange often says that a certain result holds in general, or better that certain functions have certain properties in general. This means, for him, that certain expressions can be subjected to certain transformations whenever no particular values are assigned to the variables or indeterminate quantities that occur in them, or, to use Lagrange's words, whenever these quantities remain indeterminate. For Lagrange, it is thus natural to assume that a general result concerning functions could have exceptions for particular values of some variables.

This is an intensional conception of generality, which is quite different from the extensional conception we are familiar with. According to the latter, $P$ holds in general for a certain set $S$ of individuals when $P(s)$ holds for any $s$ in $S$. According to the former, $P$ holds in general for the $s$ 's when it holds for an expression $S$ that is taken as the typical expression of the $s$ 's. ${ }^{35}$ A clear illustration of such a conception is provided by Lagrange's fundamental proof, to which we now turn.

\section{LAGRANGE's FUndAMENTAL PROOF}

We have already observed that, in order to achieve his purpose, Lagrange should have shown that derivative functions could effectively replace differential quotients. More precisely, he should have proved that the following condition holds:

\section{: [Fundamental condition of the theory of analytic functions]}

- FC. $i)$ For any function $y=f(x)$ there exist an infinity of other functions $f^{(k)}(x)(k=1,2, \ldots)$, called 'derivative functions', that are such that $\sum_{k=0}^{\infty} \frac{f^{(k)}(x)}{k !} \xi^{k}$ (where $f^{(0)}(x)=f(x)$ and $\xi$ is an indeterminate increment) is the (unique) power-series expansion of $f(x+\xi)$, and that may be uniquely determined with the same rules used in going from $f(x)$ to the corresponding differential quotients $\frac{d^{k} y}{d x^{k}}$, and therefore formally coincide with these quotients, to the effect that they are defined for any $x$ for which the differential quotients $\frac{d^{k} y}{d x^{k}}$ are.

- FC. ii) If $x_{0}$ is an isolated value of $x$ and $n$ a non-negative integer such that $\left[\frac{d^{k} y}{d x^{k}}\right]_{x=x_{0}}$ is defined if and only if $k \leq n$, then $\sum_{k=0}^{n} \frac{f^{(k)}\left(x_{0}\right)}{k !} \xi^{k}+\sum_{k=n+1}^{\infty} \digamma_{k}(\xi)$ (where $f^{(k)}(x)$ coincides with $\frac{d^{k} y}{d x^{k}}(k=0,1, \ldots, n)$ and $\digamma_{k}(\xi)$ $(k=n+1, n+2, \ldots)$ are appropriate functions of $\xi$ alone) is the (unique) expansion of $f\left(x_{0}+\xi\right)=f_{x_{0}}(\xi)$ including $n+1$ terms having respectively the form $A_{k} \xi^{k}(k=0,1, \ldots, n)$, where $A_{k}$ does not depend on $\xi$.

This condition cannot be stated as such within Lagrange's theory, and indeed it is not stated neither in the Théorie nor in the Leçons. Still, as we already observed, both these treatises open with an argument that, if correct, would have convinced anybody already familiar with the differential calculus that such a condition is met. This is Lagrange's fundamental proof. The present section is devoted to a reconstruction and evaluation of this argument.

3.1. Power-Series Expansion. The first part of Lagrange's fundamental proof is intended to establishes a result that he initially states as follows:

Theorem 1 (Power-series expansion theorem). $\quad$ Let us consider [...] a function $f(x)$ of whatever variable $x$. If, one replaces in it $x$ with $x+\xi$, where $\xi$ is whatever indeterminate quantity, it will become $f(x+\xi)$, and, for the theory of series, one will be able to expand it in a series of this form

$$
f(x)+p \xi+q \xi^{2}+r \xi^{3}+\& c .,
$$

in which the quantities $p, q, r, \& c$.- which are the coefficients of the powers of $\xi$-will be new functions of de $x$, derived from the primitive function $f(x)$, and independent of the indeterminate [quantity] $\xi$.

Considérons [...] une fonction $f(x)$ d'une variable quelconque $x$. Si à la place de $x$, on y met $x+\xi$,

$\xi$ étant une quantité quelconque indéterminée, elle deviendra $f(x+\xi)$, et par la théorie des séries on pourra la développer en une série de cette forme

$$
f(x)+p \xi+q \xi^{2}+r \xi^{3}+\& c .
$$

dans laquelle les quantités $p, q, r, \& c$., coefficients des puissances de $\xi$, seront des nouvelles fonctions de $x$, dérivées de la fonction primitive $f(x)$, et indépendantes de l'indéterminée $\xi$.

(Lagrange 1797, art. 3; 1801, p. 7; 1806, p. 8; 1813, art. I.1.) ${ }^{36}$

\footnotetext{
${ }^{35}$ Lagrange's view on this matter was shared by most of his contemporaries. In the first volume of his Traité du calcul différentiel et intégral, Lacroix observes for example that "though true in general", the form of a power series for the expansion of a function of $x+\xi$ "is not appropriate for certain particular cases" (Lacroix 1797-1798, vol. 1, p. 232).

${ }^{36}$ We correct an evident misprint of the second edition of the Théorie. In this edition, Lagrange uses the symbol '...' whenever, in the first edition of the Théorie and in both editions of the Leçon, he had used the symbol '\&c.'. We prefer to use the latter symbol, which was much commoner in te 18th century. The term 'theorem of power-series expansion' is not Lagrange's. We use it for brevity and simplicity.
} 
The reference to the theory of series requires explanation. Lagrange's intention was presumably to refer to the corpus of results expounded in the first volume of Euler's Introductio, where-by relying on appropriate formal procedures-it is showed that any rational and irrational function can be expanded in a power series, and the power-series expansions of the exponential, the logarithm, the sine and the cosine are provided. One could then think that Lagrange considered these results as a sufficient basis for proving the theorem. But this is contradicted by what he writes right away, which rather suggests that, for him, any proof merely relying on Euler's result would have been inappropriate:

But, for not to go ahead gratuitously, we begin by examining the very form of the series that should represent the expansion of any function $f(x)$ when one replaces in it $x$ with $x+\xi$, and that we have supposed to contain only integer positive powers of $\xi$. In fact, this supposition obtains for the expansion of [all] the different functions we know, but, to the best of my knowledge, nobody tried to prove it $a$ priori, which seems to me all more necessary that there are some particular cases in which it cannot obtain. ${ }^{37}$

Mais pour ne rien avancer gratuitement, nous commencerons par examiner la forme même de la série qui doit représenter le développement de toute fonction $f(x)$ lorsqu'on y substitue $x+\xi$ à la place de $x$, et que nous avons supposée ne devoir contenir que des puissances entières et positives de $\xi$. Cette supposition se vérifie en effet par le développement des différentes fonctions connues; mais personne, que je sache, n'a cherché à la démontrer a priori, ce qui me paraît néanmoins d'autant plus nécessaire, qu'il y a des cas particuliers où elle ne peut pas avoir lieu.

(Lagrange 1797, art. 10, 1801, p. 8, 1806, pp. 8-9, and 1813, art. I.2)

Such an a priori proof is precisely what the first part of Lagrange's fundamental proof is intended to provide.

In his treatises, Lagrange makes no effort to explain what it means for him that a certain series is an expansion of a certain function. In the second edition of the Théorie, after having provided his alleged a priori proof, he refers to the result allegedly established by this proof with the phrase 'la formule générale $f(x+\xi)=f(x)+p \xi+q \xi^{2}+r \xi^{3}+\& c$.' (Lagrange 1813, art. I.8; in the first edition-1797, art. 16-, the adjective 'générale' is absent). This is only one among the several occasions where Lagrange uses the symbol ' $=$ ' to denote the relation between a certain function and its powerseries expansion (or some other sort of expansions). The mere use of this symbol is, however, not enough to clarify the exact nature both of this result and of this relation. The best (and possibly only) way to achieve such a clarification is by parsing Lagrange's proof. This is what we shall do in section 3.1.2. The discussion made in this last section will be based, however, on our understanding of what Lagrange means by requiring that his proof be a priori. Before we turn to the proof it is therefore necessary to consider this last matter.

3.1.1. Why an a priori Proof? Providing functions comply with the compositional conception (explained in section 2.6, above), Euler's mentioned results on the power-series expansion of functions would be enough to prove the power-series expansion theorem. To prove that any finitary algebraic composition of the elementary functions has a power-series expansion it would be enough, indeed, to show how to obtain a power-series expansion of a composed function by composing the power-series expansions of the elementary functions that compose it. The method of indeterminate coefficients would, then, be appropriate to obtain a power-series expansion of any function implicitly defined through a (finitary) equation in two variables involving elementary functions algebraically composed. For short, call such a proof 'compositional.' It is natural to wonder why Lagrange considered it inappropriate. ${ }^{38}$

In the Introductio, Euler proves his mentioned results by appealing both to infinitesimalist arguments and to an unproved generalization of the binomial theorem to any real exponent. This is not, however, a reason that Lagrange could have advanced for requiring an alternative proof of these results based on a non-compositional proof of the power-series expansion theorem. On the one hand, the argument that he relies on in the Leçons (see pp. 25-26, below) to obtain the first coefficient of the power-series expansion of $(x+\xi)^{m}$ is a clever version of a later proof of the binomial theorem due to Æpinus and Euler himself, which works for any sort of exponent and relies on no infinitesimalist assumptions (Æpinus 1760-1761; Euler 1787; Dhombres 1987; Dhombres and Pensivy 1988; Pensivy 1987-1988.). On the other hand, when he looks for the first coefficients of the power-series expansions of $a^{(x+\xi)}, \log _{a}(x+\xi), \sin (x+\xi)$, and $\cos (x+\xi)$, both in the Théorie and in the Leçons (Lagrange 1797, arts. 19-23 and 25-29; 1801, pp. 20-24 and 31-35; 1806, pp. 25-3; pp. 40-47; 1813, arts. I.11-14 and I.18-I.19; Panza 1992, pp. 749-760), Lagrange offers arguments that are perfectly suitable to provide, independently of all infinitesimalist considerations, the very power-series expansion of the exponential, the logarithm, the sine and the cosine obtained by Euler. Lagrange could thus have easily obtained Euler's results through arguments that he would certainly have considered correct, and that are independent of the power-series expansion theorem.

One can wonder, then, why, rather than proceeding accordingly, he sought a non-compositional (a priori) proof. Three explanations come to mind.

\footnotetext{
${ }^{37}$ For an explanation of this last disclaimer, see p 19, below.

${ }^{38}$ Lagrange's phrase 'pour ne rien avancer gratuitement', included in one of the passages quoted above, makes it hard to argue that Lagrange merely preferred an a priori proof but did not consider a compositional one inappropriate.
} 
The first is as follows. Let $F(x, y)=0$ a (finitary) equation involving Lagrange's elementary functions algebraically composed, and $f(x+\xi)$ the function implicitly defined through the corresponding equation $F(x+\xi, y)=0$. To develop this function into a power-series expansion by relying on the method of indeterminate coefficients, one has to suppose that $f(x+\xi)=\sum_{k=0}^{\infty} A_{k} \xi^{k}$, then apply this method to determine the coefficients $A_{k}$ in terms of $x$. It follows that either it is taken for granted that any function $f(x+\xi)$ so defined reduces to a finitary algebraic composition of Lagrange's elementary functions, or a compositional proof of the power-series expansion theorem is in some way circular, since the equality $f(x+\xi)=\sum_{k=0}^{\infty} A_{k} \xi^{k}$ could only be justified by the assumption that $f(x+\xi)$ has a power-series expansion. It is thus possible that Lagrange required a non-compositional proof of the power-series expansion theorem to avoid assuming that any function $f(x+\xi)$ so defined reduces to a finitary algebraic composition of its elementary functions.

The second explanation is simpler. It could be that Lagrange wanted a non-compositional proof of the power-series expansion theorem to avoid making another assumption (that a compositional proof would have required): the assumption that power series obtained by appropriately composing the power-series expansions of elementary functions is a powerseries expansion of the function obtained by analogously composing these same elementary functions.

The third explanation is radical. Perhaps Lagrange wanted a non-compositional proof of the power-series expansion theorem because he wanted the proof of this crucial theorem to work even independently of the adoption of the compositional conception of functions. ${ }^{39}$

All these explanations indicate a discrepancy between Lagrange's actual proof of the power-series expansion theorem and his ideal of purity, as they suggest that Lagrange sought a proof that does not depend on assumptions concerning functions that are natural to make if functions are conceived as expressions according to the view explained in section 2. They suggest, moreover, that what Lagrange means by an a priori proof of this theorem is a proof that does not rely on formal procedures like Euler's: procedures leading to the power-series expansion of certain functions through appropriate transformations of the relevant expression.

3.1.2. The Premises of Lagrange's Proof of the Power-Series Expansion Theorem and the Appropriate Understanding of this Theorem. We are now ready to consider Lagrange's a priori proof of the power-series expansion theorem. This is far from unequivocal, as has often been noticed. We will therefore quote it in extenso, before parsing it:

I shall firstly prove that, in the series resulting from the extension of the function $f(x+\xi)$, there cannot be any fractional power of $\xi$, unless one assigns to $x$ some particular values.

It is clear, indeed, that the radicals of $\xi$ can only result from some radicals included in the primitive function $f(x)$, and it is also clear that replacing $x$ with $x+\xi$ could neither increase nor decrease the number of these radicals, and it could no more change their nature, as far as $x$ et $\xi$ are indeterminate quantities. Furthermore, from the theory of equations, we know that any radical has as many different values as there are unities in its exponent, and any irrational function has then as many different values as possible combinations of the different values of the radicals it includes. Hence, if the expansion of the function $f(x+\xi)$ were able to contain a term of the form $u \xi^{\frac{m}{n}}$, the function $f(x)$ would necessarily be irrational and would then have a certain number of different values, which would be the same for the function $f(x+\xi)$ as for its expansion. But, since this expansion is represented by the series

$$
f(x)+p \xi+q \xi^{2}+\& c .+u \xi^{\frac{m}{n}}+\& c .
$$

\footnotetext{
${ }^{39}$ In the first edition of the Leçons, Lagrange takes his proof to be concerned only with "algebraic functions" and makes, at the end of it, the following quite peculiar remark:

If the function $f(x)$ is not algebraic, one can nevertheless suppose that the expansion of $f(x+\xi)$ be, in general, of this same form, regarding as particular exceptions the cases where this expansion would contain some powers of $\xi$ other than the integer positive ones. Thus, whatsoever the function $f(x)$ might be, we only consider the functions $p, q, r, \& c$. that result from the expansion of $f(x+\xi)$ according to the powers $\xi, \xi^{2}, \xi^{3}, \& c$.

Si la fonction $f(x)$ n'est pas algébrique, on peut néanmoins supposer que le développement de $f(x+\xi)$ soit en général de cette même forme, en regardant comme des exceptions particulières les cas où ce développement contiendrait d'autres puissances de $\xi$ que des puissances positives et entières. Ainsi, quelle que soit la fonction $f(x)$ nous ne considérerons que les fonctions $p$, $q, r, \& c$. résultant du développement de $f(x+\xi)$ suivant les puissances $\xi, \xi^{2}, \xi^{3}, \& c$.

(Lagrange 1801 , pp. 9-10.)
}

This remark, together with the restriction to algebraic functions, are absent both from the two editions of the Théorie and from the second edition of the Leçons. It isn't clear what sort of exceptions Lagrange had in mind. So it is hard to decide whether this remark should be taken as evidence that Lagrange sought a proof of the power-series expansion theorem that worked independently of the adoption of the compositional conception of functions. 
each value of $f(x)$ would combine with each one of the $n$ values of the radical $\sqrt[n]{\xi^{m}}$, to the effect that the function $f(x+\xi)$ would have, when it is expanded, more different values than this same function when it is not expanded, which is absurd.

This proof is general and rigorous as far as $x$ and $\xi$ remain indeterminate; but it would cease to be so if one assigned to $x$ some determinate values, since it would be possible that these values destroy some radicals in $f(x)$ that could nevertheless subsist in $f(x+\xi)$. [...]

We have just seen that the expansion of the function $f(x+\xi)$ could not include, in general, some fractional powers of $\xi$; it is also possible to warrant that it could no more include some negative powers of $\xi$.

Since, if among the terms of this expansion, there were one of the form $\frac{r}{\xi^{m}}$, where $m$ is a positive number, this term would become infinite; thus the function $f(x+\xi)$ should also become infinite when $\xi=0$; and consequently $f(x)$ should become infinite, which could only happen for some particular values of $x$.

Je vais d'abord démontrer que, dans la série résultante du développement de la function $f(x+\xi)$, il ne peut se trouver aucune puissance fractionnaire de $\xi$, à moins qu'on ne donne à $x$ des valeurs particulières.

En effet, il est clair que les radicaux de $\xi$ ne pourraient venir que des radicaux renfermés dans la fonction primitive $f(x)$, et il est clair en même temps que la substitution de $x+\xi$ au lieu de $x$ ne pourrait ni augmenter ni diminuer le nombre de ces radicaux, ni en changer la nature, tant que $x$ et $\xi$ sont des quantités indéterminées. D'un autre côté, on sait par la théorie des équations que tout radical a autant de valeurs différentes qu'il y a d'unités dans son exposant, et que toute fonction irrationnelle a, par conséquent, autant de valeurs différentes qu'on peut faire de combinaisons des différentes valeurs des radicaux qu'elle renferme. Donc, si le développement de la fonction $f(x+\xi)$ pouvait contenir un terme de la forme $u \xi^{\frac{m}{n}}$, la fonction $f(x)$ serait nécessairement irrationnelle et aurait par conséquent un certain nombre de valeurs différentes, qui serait le même pour la fonction $f(x+\xi)$, ainsi que pour son développement. Mais, ce developpement étant représenté par la série

$$
f(x)+p \xi+q \xi^{2}+\& c .+u \xi^{\frac{m}{n}}+\& c .,
$$

chaque valeur de $f(x)$ se combinerait avec chacune des $n$ valeurs du radical $\sqrt[n]{\xi^{m}}$, de sorte que la fonction $f(x+\xi)$ développée aurait plus de valeurs différentes que la même fonction non développée, ce qui est absurde. Cette démonstration est générale et rigoureuse tant que $x$ et $\xi$ demeurent indétérminés; mais elle cesserait de l'être si l'on donnait à $x$ des valeurs détérminées, car il serait possible que ces valeurs détruisissent quelques radicaux dans $f(x)$ qui pourraient néanmoins subsister dans $f(x+\xi)$. [...]

Nous venons de voir que le développement de la fonction $f(x+\xi)$ ne saurait contenir, en général, des puissances fractionnaires de $\xi$; il est facile de s'assurer aussi qu'il ne pourra contenir non plus des puissances négatives de $\xi$.

Car, si parmi les termes de ce développement, il y en avait un de la forme $\frac{r}{\xi^{m}}, m$ étant un nombre positif, en faisant $\xi=0$, ce terme deviendrait infini; donc la fonction $f(x+\xi)$ devrait devenir infinie lorsque $\xi=0$; par conséquent, il faudrait que $f(x)$ devînt infinie, ce qui ne peut avoir lieu que pour des valeurs particulières de $x$.

(Lagrange 1797, art. 10; 1801, pp. 8-9; 1806, pp. 9-10; 1813, art. I.2; the second part of the quotation, concerned with negative powers of $\xi$, is absent from the first edition of the Théorie.)

If this argument is taken to provide the complete proof of the power-series expansion theorem, as we suggest, ${ }^{40}$ it is based (among others) on an unproved premise. For brevity, let us say that a series $S(x, \xi)$ is a generalized power series if it is of the form $\sum_{k=0}^{\infty} p_{k}(x) \xi^{\alpha_{k}}$, where the coefficients $p_{k}(x)$ are functions of $x$ and the exponents $\alpha_{k}$ are rational (and, of course, different from each other). ${ }^{41}$ Lagrange's unproved premise can then be stated as follows:

- [P] Any function $f(x+\xi)$ has a generalized power-series expansion. ${ }^{42}$

This is not, of course, the only premise on which Lagrange's proof of the power-series expansion theorem rests. Three others premises are the following:

- [C] For any function $f(x+\xi)$ and any generalized power series $S(x, \xi)$, if the latter is an expansion of the former then:

- $i$ ) if $S(x, \xi)$ contains a radical of $\xi$ then $f(x)$ is also irrational;

- ii) $f(x+\xi)$ and $S(x, \xi)$ have the same number of values; ${ }^{43}$

\footnotetext{
${ }^{40}$ In section 5.2.1, pp. 42-43, we shall consider an alternative understanding of Lagrange's proof of the power-series expansion theorem, according to which this argument provides only the first part of this proof.

${ }^{41}$ In what follows, we shall denote with ' $p_{1}(x)$ ', ' $p_{2}(x)$ ', ' $p_{3}(x)$ ', $\ldots$ the functions Lagrange denotes with ' $p$ ', ' $q$ ', ' $r$ ', ... We also use the modern symbol ' $\sum_{k=0}^{\infty}$-' to denote a series. Lagrange writes instead the first terms of this series followed by the symbols '\&c.' or '...' (see footnote (36), above). These last symbols clearly indicate, in his treatises, that the sum is indefinitely, rather than infinitely, extended. The reader is thus invited to consider the possibility that all terms of a series vanish beyond a certain value of $k$.

${ }^{42}$ For the case where $f(x+\xi)$ has an expansion including some powers of $\log \xi$, see section 3.4, especially pp. 30-3.4, below.

${ }^{43}$ We shall return to this premise, and try to shed light on it, in section 3.1.3.
} 
- iii) if $S(x, \xi)$ becomes infinite for $\xi=0$, then $f(x)$ is also infinite.

In the proof we have just quoted, Lagrange justifies condition [C. $i$ ] by claiming that "the radicals of $\xi$ [in $S(x, \xi)$ ] could only come from radicals contained in the [...] function $f(x)$ ". In the Leçons, he also argues that "the form" of the functions $p_{k}(x)(k=0,1,2, \ldots)$ "depends only on that of the given function $f(x)$ " and adds that these functions "can be easily determined, in the particular cases, through the rules of ordinary algebra" (Lagrange 1801, p. 7; 1806, p. 8).

At first glance, these claims suggest that, for Lagrange, to say that a certain generalized power series is an expansion of a certain function is the same as saying that the former results from the latter through the application of an appropriate formal procedure. This is consistent with Lagrange's understanding of functions as expressions, but neither with the $a$ priori character of the desired proof, nor with conditions [C.ii] and [C.iii].

These two last conditions suggest, rather, an alternative understanding, which is not only consistent with the $a$ priori character of the proof and these same conditions, but also with Lagrange's understanding of functions as quantities, and the mathematical practice concerning expansion of functions that was usual within algebraic analysis before Lagrange (Ferraro and Panza 2003, especially sections 2 and 3). This understanding is a general (or global) one in the following sense: a generalized power series $S(x, \xi)$ counts as an expansion of a function $f(x+\xi)$ insofar as both this series and this function are understood as expressions where $x$ and $\xi$ remain indeterminate, that is, as expressions taken as such (or expressions where $x$ and $\xi$ are not supposed to take any particular value).

Let us fix this understanding through an explicit definition:

Definition 1. A generalized power series $S(x, \xi)$-where $x$ and $\xi$ remain indeterminate-is an expansion of a function $f(x+\xi)$-where $x$ and $\xi$ remain indeterminate as well-if and only if, for any value of $x$ for which this series is defined, it converges ${ }^{44}$ to such a function whenever the value of $\xi$ belongs to an appropriate proper interval. ${ }^{45}$

The following considerations should be enough to show how this understanding works in Lagrange's theory and to explain it better.

Though general in the previous sense, this definition is such that, according to it, a function $f(x+\xi)$ has a generalized power-series expansion $S(x, \xi)$ in virtue of what happens under appropriate replacements of $x$ and $\xi$ with constant quantities. This is just the sort of understanding that is needed both for the power-series expansion theorem and for premise $[\mathbf{P}]$, since-we argue - both of them are supposed to be general in this same sense.

Definition 1 suggests that premise $[\mathbf{P}]$ be understood as follows:

- [P.1] For any function $f(x+\xi)$-where $x$ and $\xi$ remain indeterminate-there is a generalized power series $S(x, \xi)$-where $x$ and $\xi$ remain indeterminate, in turn-such that, for any value of $x$ for which it is defined, it converges to $f(x+\xi)$ whenever the value of $\xi$ belongs to an appropriate proper interval.

However, this understanding of premise $[\mathbf{P}]$ is too weak for justifying Lagrange's use of this premises in his proof of the power-series expansion theorem. Let us see why.

Having admitted premise [P], Lagrange is satisfied with two reductio ad absurdum purporting to exclude the possibility that a generalized power-series expansion of a function $f(x+\xi)$ contains fractional and negative powers of $\xi$, respectively.

The first reductio, intended to exclude fractional exponents, starts with the supposition that this function has a generalized power series expansion of the form

$$
f(x)+\ldots+p_{\mu}(x) \xi^{\frac{m}{n}}+\& c .,
$$

where $\mu$ is a positive integer greater than 0 , and $\frac{m}{n}$ is a fractional exponent (different from every other exponent of $\xi$ in this expansion). In section 3.1.3, we shall return to the argument that Lagrange develops starting from this supposition. For the time being, it is only relevant to remark that, according to such a supposition, this expansion includes the function $f(x)$ as one of its terms. This is essential, since the reductio proceeds by showing that the generalized power series expansion of $f(x+\xi)$ cannot contain both $f(x)$ and $p_{\mu}(x) \xi^{\frac{m}{n}}$, and then, as it certainly contains the former, it cannot contain the letter. Now, if definition 1 is accepted, $f(x)$ would enter a generalized power series expansion of $f(x+\xi)$ if no exponent of $\xi$ in this expansion were negative, since this would ensure that the proper interval to which $\xi$ must belong for this expansion to converge to $f(x+\xi)$ includes the value $\xi=0$. One could then think that the second reductio-which is primarily intended to exclude negative exponents-is also suitable for proving that the (or any) generalized power series expansion of a function $f(x+\xi)$ includes the function $f(x)$ as one of its terms.

Still, this second reductio proceeds by making the other assumption that, whatever $x$ might be, if a generalized powerseries expansion of $f(x+\xi)$ goes to infinity for $\xi=0$, this also happens for $f(x+\xi)$ itself. This is the same as assuming that this expansion converges to $f(x+\xi)$ for $\xi=0$, to the effect that $S(x, 0)=f(x)$. This is precisely what happens for

\footnotetext{
${ }^{44}$ As the purpose of definition 1 is to fix the way Lagrange understands the relation of a function and a generalized power-series expansion of it, the convergence of the former to the latter has to be understood as Lagrange could have understood it, that is, as a not-better-defined metric equality between the values of the function and the values that the series indefinitely approaches. In what follows, we shall always use the verb 'to converge' and its cognates in this same quite vague sense, which corresponds, we argue, to the understanding of eighteen-century mathematicians. For further considerations on this matter, see Ferraro and Panza (2003).

${ }^{45} \mathrm{By}$ 'proper interval' we mean one that includes more than a single value.
} 
power series. Thus, either Lagrange's second reductio is circular in some way, or it depends on the assumption that a generalized power-series expansion of $f(x+\xi)$ converges to $f(x+\xi)$ around $\xi=0$. Hence, it can neither provide a proof for this last assumption, nor for the assumption that such an expansion includes $f(x)$ as one of its terms.

It thus appears that Lagrange takes this last assumption for granted. In other words, he takes it for granted that a generalized power series of a function $f(x+\xi)$ is a series that converges to this function for small enough $\xi$, and merely proves that this is possible only of if this expansion is a power series.

Now, the requirement that a generalized power series of a function $f(x+\xi)$ converge to this function for small enough $\xi$ and include then he function $f(x)$ as one of its terms is certainly relevant for Lagrange's proof to hold, but it is not relevant to the content of this last theorem. Indeed, a power series $\sum_{k=0}^{\infty} p_{k}(x) \xi^{k}$ converges if and only if $\xi$ belongs to an interval containing zero, ${ }^{46}$ and it can thus converge to $f(x+\xi)$ only if it includes $f(x)$ as one of its terms. A natural way to understand this theorem is thus the following:

Theorem 2. For any function ${ }^{47} f(x+\xi)$-where $x$ and $\xi$ remain indeterminate-, there is a power series $\sum_{k=0}^{\infty} p_{k}(x) \xi^{k}$ where $x$ and $\xi$ remain indeterminate as well, and the coefficients $p_{k}(x)$ are functions of $x$-that, for any value of $x$ for which it is defined, converges to such a function whenever the value of $\xi$ belongs to an appropriate proper interval centered on zero.

Suppose now that $\sum_{k=0}^{\infty} p_{k}(x) \xi^{k}$ be a power-series expansion of a certain function $f(x+\xi)$. Suppose also that $x_{0}$ be a value of $x$ for which $\sum_{k=0}^{\infty} p_{k}\left(x_{0}\right) \xi^{k}$ is not defined. Insofar as $\xi$ remains indeterminate when $x$ takes the value $x_{0}$ in $f(x+\xi)$, this function reduces to a function of $\xi$ alone, namely $f\left(x_{0}+\xi\right)=f_{x_{0}}(\xi)$. Theorem 2 is clearly compatible with the possibility that this last function have a generalized power-series expansion $\sum_{k=0}^{\infty} p_{k}\left(x_{0}\right) \xi \alpha_{k}$ which is not a power series.

Two simple examples are provided by the functions $\frac{1}{x+\xi}$ and $\sqrt{x+\xi}$ and the respective power-series expansions $\sum_{k=0}^{\infty}(-1)^{k} \frac{1}{x^{k+1}} \xi^{k}$ and $\sum_{k=0}^{\infty}\left(\begin{array}{c}\frac{1}{2} \\ k\end{array}\right) \frac{\xi^{k}}{x^{k-1} \sqrt{x}}$. The former of these series is not defined for $x=0$, the latter is not defined for $x \leq 0$. For these values of $x$, the functions $\frac{1}{x+\xi}$ and $\sqrt{x+\xi}$ reduce to $\frac{1}{\xi}$ and $\sqrt{a+\xi}$ (where $a$ is a non-positive constant), respectively. The former of these last functions is then its own generalized power-series expansion, whereas the latter has $\sum_{k=0}^{\infty}\left(\begin{array}{c}\frac{1}{2} \\ k\end{array}\right) \frac{a^{k}}{\xi^{k-1} \sqrt{\xi}}$ as its generalized power-series expansion. ${ }^{48}$

Even if theorem 2 holds, there is thus a sense in which a function $f(x+\xi)$ can have a generalized power-series expansion that is not a power series. This explains what Lagrange means when he remarks (at the end of the passage quoted at page 15, after theorem 1), that "there are some particular cases where [...][the supposition that the expansion of $f(x+\xi)$ contains only integer positive powers of $\xi$ ] cannot hold".

As Lagrange says in his proof, these cases are given by "particular values of $x$ ". The term 'particular values' suggests that Lagrange is here thinking to isolated values on an appropriate domain.

Plausibly, this domain includes the domain of definition of $f(x)$, but it seems that Lagrange also considers as being relevant the limit values of this domain that, though not being included in it, allow the function $f(x+\xi)$ to be defined for any non-null value of $\xi$. In other terms, it seems that he takes the relevant "particular values of $x$ " to be isolated values in the domain of definition of $f(x+\xi)$ for any non-null value of $\xi .{ }^{49}$ For example, in the case of the function $\frac{1}{x}$, the relevant

\footnotetext{
${ }^{46}$ In Lagrange's day, nobody had spotlessly proved that this is the case. In the 18th century, this was, however, generally taken for granted (though nobody remarked that the interval of convergence could, in some cases, reduce to the only value $\xi=0$ ), and we can suppose that this was also the case for Lagrange.

${ }^{47}$ According to Desanti 1973, p. 66, Lagrange's theory includes "a principle of 'normalisation' which delimits a priori the class of functions that can be expanded in series". This seems wrong to us. We maintain, rather, that Lagrange wanted to prove that any function of $x+\xi$ has a power-series expansion, at least if this claim is appropriately understood.

${ }^{48}$ Though the power series $\sum_{k=0}^{\infty}\left(\begin{array}{c}\frac{1}{2} \\ k\end{array}\right) \frac{\xi^{k}}{x^{k-1} \sqrt{x}}$ is not defined for $x=0$, there is a generalized power series that provides an expansion of $\sqrt{x+\xi}$ and is defined for any value of $x$ for which $\sqrt{x}$ is defined, and thus also for $x=0$. This is $\sum_{k=0}^{\infty}\left(\begin{array}{c}\frac{1}{2} \\ k\end{array}\right) \frac{x^{k}}{\xi^{k-1} \sqrt{\xi}}$. But this is a power series of $x$, not of $\xi$. It does not contain the function $\sqrt{x}$ as one of its terms and does thus appear not to count, for Lagrange, as a generalized power-series expansion of $\sqrt{x+\xi}$, insofar as this function results from the function $\sqrt{x}$ by replacing $x$ with $x+\xi$. It rather counts as a generalized power-series expansion of the function $\sqrt{\xi+x}$, insofar as this function results from the function $\sqrt{\xi}$ by replacing $\xi$ with $\xi+x$. Conceived as resulting from $\sqrt{x}$ by replacing $x$ with $x+\xi$, the function $\sqrt{x+\xi}$ has thus only a generalized power-series expansions, namely, $\sum_{k=0}^{\infty}\left(\begin{array}{c}\frac{1}{2} \\ k\end{array}\right) \frac{\xi^{k}}{x^{k-1} \sqrt{x}}$.

${ }^{49}$ This seems to be also Dugac's view: see Dugac (2003), p. 73, where he claims that Lagrange takes the relevant particular cases to be given by a finite number of points on a closed interval.
} 
domain seems also to include the value $x=0$, for which this function is not defined (since $\frac{1}{x+\xi}$ is defined for $x=0$ if the increment $\xi$ is non-null), while, in the case of the function $\sqrt{x}$, the only "particular value" to be considered is $x=0$.

The consideration of the limit values of the domain of definition of $f(x)$ which are not included in this domain is however mathematically nonessential for Lagrange's theory, and rather depends on rhetoric reasons: to say that the powerseries expansion of $f(x+\xi)$ is not defined for these values simply reduces to say, within this theory, that the function $f(x)$ has not derivatives for some values of $x$ for which it is not defined. Hence, in what follows, we shall limit ourselves to consider the "particular values of $x$ " to be isolated values in the domain of definition of $f(x)$.

A little reflection clarifies the situation. Let $\sum_{k=0}^{\infty} p_{k}(x) \xi^{k}$ and $\sum_{k=0}^{\infty} \hat{p}_{k}(x) \xi^{k}$ be two distinct power-series defined on the same interval of values of $x$. Suppose that, on this interval, both of them converge to a function $f(x+\xi)$ whenever $\xi$ belongs to an appropriate proper interval centered on zero. Then, there would be a proper interval of values of $\xi$ for which, on the mentioned interval of values of $x, \sum_{k=0}^{\infty} p_{k}(x) \xi^{k}=\sum_{k=0}^{\infty} \hat{p}_{k}(x) \xi^{k}$. For the method of indeterminate coefficients, it would then follow that $p_{k}(x)=\hat{p}_{k}(x)$ for any $k$, which contradicts the assumption that $\sum_{k=0}^{\infty} p_{k}(x) \xi^{k}$ and $\sum_{k=0}^{\infty} \hat{p}_{k}(x) \xi^{k}$ are distinct. Hence, if the method of indeterminate coefficients is accepted, theorem 2 excludes the possibility that a function $f(x+\xi)$ have distinct power-series expansions defined on a same interval of values of $x$. But it leaves open both the possibility that a function $f(x+\xi)$ have several distinct power series expansions, each of which is defined for different intervals in the domain of definition of $f(x)$, and the possibility that a function $f(x+\xi)$ admit no power-series expansion defined for some proper interval in this same domain of definition.

The former possibility would undermine, however, the second part of Lagrange's fundamental proof, which we shall discuss in section 3.2. Moreover, his use of the terms 'determined values' and 'particular values,' and his treatment of the cases corresponding to these values, suggest that he does not consider the latter.

Insofar as his proof of the power-series expansion theorem does not eliminate these two possibilities, it is natural to think that their exclusion depends on his understanding of premise $[\mathbf{P}]$. So, in the end, it seems to us that, to adhere to Lagrange's views, this premise and this theorem should be, respectively, understood as follows:

- [P.2] For any function $f(x+\xi)$-where $x$ and $\xi$ remain indeterminate-there is a generalized power series $S(x, \xi)$ - where $x$ and $\xi$ remain indeterminate as well-which includes $f(x)$ as one of its terms, and is defined for any $x$ in the domain of definition of $f(x)$ except for some isolated values, and that, for any value of $x$ for which it is defined, converges to $f(x+\xi)$ whenever the value of $\xi$ belongs to an appropriate proper interval centered on zero.

Theorem 3. This generalized power series is necessarily a power series and it is thus unique. In other words, for any function $f(x+\xi)$-where $x$ and $\xi$ remain indeterminate—, there is one and only one power series $\sum_{k=0}^{\infty} p_{k}(x) \xi^{k}$-where $x$ and $\xi$ remain indeterminate as well, and the coefficients $p_{k}(x)$ are functions of $x$-that is defined for any $x$ in the domain of definition of $f(x)$ except for some isolated values, and that, for any value of $x$ for which it is defined, converges to such a function whenever the value of $\xi$ belongs to an appropriate proper interval centered on zero. ${ }^{50}$

If the power-series expansion theorem is so understood, there are no easy counter-examples to it in the universe of Lagrange's functions, ${ }^{51}$ at least if his notion of function is understood strictu sensu.

If this is the case, Cauchy's counter-examples - consisting of functions of the form $e^{-1 / g(x)}$, where $g(x)$ is defined for any (real) value of $x$, is indefinitely derivable at the origin, and is such that $g(0)=0$ and $g(x)>0$ for $x \neq 0$ (Cauchy 1822 , p. $277-278 ; 1823$, p. 152$)^{52}$ - do not apply. It is true that, if one is allowed to extend these functions and their derivatives continuously to $x=0$, the power-series expansions of $e^{-1 / g(x+\xi)}$ are defined for $x=0$, but reduce in this case to $0+0+0+\ldots$, which is obviously not equal to $e^{-1 / g(0+\xi)}=e^{-1 / g(\xi)}$. Extending a function continuously to a point in which it is not defined is however the same as replacing this function with a piece-wise defined function, which is not allowed, in agreement Lagrange's notion of function understood strictu sensu.

\footnotetext{
${ }^{50}$ Note that this theorem is equivalent neither to the statement that for any function $f(x)$ and any value $x_{0}$ in its domain of definition there is a series $\sum_{k=0}^{\infty} p_{k}(a)(x-a)^{k}$ which converges to $f(x)$ whenever $x$ belongs to a proper interval centered on $x=a$ that includes $x_{0}$, nor to the statement that any value $x_{0}$ of the domain of definition of any function $f(x)$ can be chosen as the centre of an expansion of the form $\sum_{k=0}^{\infty} p_{k}\left(x_{0}\right)\left(x-x_{0}\right)^{k}$ for this function.

${ }^{51}$ According to Grabiner (1990, p. 15]), "it is not true that any function given by an analytic expression can be expressed as the sum of a convergent Taylor series about any arbitrary point. (It was not sufficient for Lagrange to except a finite number of isolated points at which the function or its derivative cease to exist.)" Unfortunately, Grabiner does not illustrate her claim with any particular example, and it is not clear how exactly this claim should be understood.

${ }^{52}$ In (1822), Cauchy considers the functions $e^{-1 / x^{2}}, e^{-1 / \sin ^{2} x}, e^{-1 / x^{2}\left(a+b x+x^{2}+\ldots\right)}$ and $e^{-1 / x}$, supposing that $x$ takes only positive values. In (1823), he considers only the function $e^{-1 / x^{2}}$.
} 
In the case of functions of the form $e^{-1 / g(x)}$, extending them continuously to $x=0$ is the same as replacing them with piece-wise defined functions of the form $f(x)=\left\{\begin{array}{cc}e^{-1 / g(x)} & x \neq 0 \\ 0 & x=0\end{array}\right.$. Hence, in agreement with Lagrange's notion of function understood strictu sensu, the value $x=0$ is such that the power-series series expansion of any function $e^{-1 / g(x)}$ is not defined for it. ${ }^{53}$

There is no doubt, however, that this point belongs to the domain for definition of $f(x+\xi)=e^{-1 / g(x+\xi)}$, for any non-null value of $\xi$. One could then argue that Lagrange should have taken this value to be a particular value of $x$ for which the power-series expansion of this last function is not defined. This is all the more likely that, when Lagrange deals with the particular values of $x$ for which the power-series expansion of a function $f(x+\xi)$ is not defined, he takes into account the possibility that a function $f\left(x_{0}+\xi\right)=f_{x_{0}}(\xi)$ have an expansion including some power of log $\xi$, and, in order to manage this case, he seems to implicitly admit that a function could be extended continuously to a value of its variable for which it is not defined as such. ${ }^{54}$ We shall return to this matter in section 3.4. Here, it is only important to observe that , if this were admitted, Cauchy's counter-examples would work. This is, however, more a case against the appropriateness of Lagrange's notion of function, than a genuine case against theorem $3 .^{55}$

Similar considerations apply to the function resulting from the series $\sum_{k=0}^{\infty}(-1)^{k} k ! \xi^{k+1}$. Though this series only converges for $\xi=0$, it can be understood as the power-series expansion of the function $e^{\frac{1}{\xi}} \int_{0}^{\xi} \frac{e^{-\frac{1}{t}}}{t} d t$, since the former can be obtained from the latter through reiterated integrations by parts (Ovaert 1976, p. 182). ${ }^{56}$ By replacing $x$ with $x+\xi$ and expanding the integer powers of this last binomial, one obtains the power series $\sum_{k=0}^{\infty}\left(\sum_{h=0}^{\infty}(-1)^{h} h !\left(\begin{array}{c}h+1 \\ k\end{array}\right) x^{h+1-k}\right) \xi^{k}$. This series can thus be taken to be the power series-expansion of the function $e^{\frac{1}{x+\xi}} \int_{0}^{x+\xi} \frac{e^{-\frac{1}{t}}}{t} d t$, despite the fact that both

\footnotetext{
${ }^{53}$ Dugac (2003, p. 77) has argued that Lagrange "did not believe in the existence" of counter-examples like Cauchy's. For Dugac, evidence for this claim is provided by the following quotation:

One should not fear that the functions $f(x), f^{\prime}(x), f^{\prime \prime}(x), \& c$. [ . . ], up to the infinity, could vanish all together under the supposition that $x=a$, as some geometers seem to suppose.

Il n'est pas à craindre que les fonctions $f(x), f^{\prime}(x), f^{\prime \prime}(x), \&$ c. [...] à l'infini puissent devenir nulles en même temps par la supposition $x=a$, comme quelques géomètres paraissent le supposer $[\ldots]$.

(Lagrange 1797, art. 39; 1813, art. I.28; see also 1801, p. 62; 1806, p. 83.)
}

From Lagrange's point of view, this claim seems however not to be relevant for the case of Cauchy's counter-examples. For him, derivatives are defined through power-series expansions, so they could all be equal to 0 for $x=a$ only if the power series expansion of $f(x+\xi)$ were defined for $x=a$ and, for this value of $x$, would converge to $f(a+\xi)$ whenever $\xi$ belongs to an appropriate proper interval. In the lines that follow the previous quotation he argues, indeed, that if it were the case that $f(x)=f^{\prime}(x)=f^{\prime \prime}(x)=\ldots=0$ for $x=a$, then it would also be the case that $f(a+\xi)=0$ for any $\xi$, which, he says, is impossible. Lagrange is thus supposing that $f(x+\xi)$ has a power-series expansion that, for $x=a$, converges to $f(a+\xi)$, which is not the case for $f(x)=\left\{\begin{array}{cc}e^{-1 / g(x)} & x \neq 0 \\ 0 & x=0\end{array}\right.$ with $a=0$.

${ }^{54}$ One could also account for the argument Lagrange advances in this case by admitting that the value of a function could be determined through a calculation involving the infinite. If this were licensed, one could argue that $\left[e^{-1 / g(0)}\right]=e^{-1 / 0}=e^{-\infty}=0$, and $\left[e^{-1 / g(0)}\right][g(0)]^{-v}=e^{-1 / 0} 0^{v}=$ $e^{-\infty} \infty=0(v=1,2, \ldots)$, which would allow to conclude that both a function of the form $e^{-1 / g(x)}$ and all its derivatives take the value 0 for $x=0$, and are thus defined for this value.

${ }^{55}$ Note that the behavior of a function of the form $f(x)=\left\{\begin{array}{cl}e^{-1 / g(x)} & x \neq 0 \\ 0 & x=0\end{array}\right.$ for $x=0$ does not prevent us from associating with it a power series which, for any $x$ different from 0 , converges to $f(x+\xi)$ whenever the value of $\xi$ belongs to an appropriate proper interval centered on zero. To take a simple example suppose that $g(x)=x^{2}$. According to the binomial theorem for negative integer exponents and the expansion of the exponential function, we have:

$$
e^{-(x+\xi)^{-2}}=e^{-x^{-2}}+2 e^{-x^{-2}} x^{-3} \xi+e^{-x^{-2}}\left[2 x^{-6}-3 x^{-4}\right] \xi^{2}+\& c .
$$

which for non-vanishing $x$ poses no problem.

${ }^{56}$ Ovaert claims that the series $\sum_{k=0}^{\infty}(-)^{k} k ! \xi^{k+1}$ is a counter-example to Lagrange's remainder theorem. According to him, the proof that this series is the power-series expansion of $e^{\frac{1}{\xi}} \int_{0}^{\xi} \frac{e^{-\frac{1}{t}}}{t} d t$ was given by Lacroix, who would not appear to have remarked, however, that his result is a counter-example to Lagrange's theorem. This would have been later proved by Laguerre. Lacroix (1800, pp. 372; 1810-1819, vol. III, p. 347) shows, indeed, with quite a complex argument, that $e^{\frac{1}{\xi}} \int \frac{e^{-\frac{1}{t}}}{t} d t$ can be taken as the "expression of the limit of the series" $\sum_{k=0}^{\infty}(-)^{k} k ! \xi^{k+1}$. Laguerre $(1878-1879)$ shows, instead, that the formal equality $e^{\frac{1}{\xi}} \int_{0}^{\xi} \frac{e^{-\frac{1}{t}}}{t} d t=\sum_{k=0}^{\infty}(-)^{k} k ! \xi^{k+1}$ can be easily obtained by calculating by parts the integral $\int_{z}^{\infty} \frac{e^{-y}}{y} d y$, so as to obtain, for any non-negative integer $h$,

$$
\int_{z}^{\infty} \frac{e^{-y}}{y} d y=e^{-z}\left[\sum_{k=0}^{h-1}(-)^{k} \frac{k !}{z^{k+1}}\right]+(-)^{h} h ! \int_{z}^{\infty} \frac{e^{-y}}{y^{h+1}} d y,
$$

then supposing that $h$ goes to infinity, and effecting the substitutions $y \rightarrow \frac{1}{t}$ and $z \rightarrow \frac{1}{\xi}$. On Lacroix's and Laguerre's results, see Borel (1929), pp. 8 and $55-56$. 
this series and the function $e^{\frac{1}{x}} \int_{0}^{x} \frac{e^{-\frac{1}{t}}}{t} d t$ are defined for any value of $x$ different from zero and the former never converges to $e^{\frac{1}{x+\xi}} \int_{0}^{x+\xi} \frac{e^{-\frac{1}{t}}}{t} d t$.

Hence, if one conceded that $e^{\frac{1}{x}} \int_{0}^{x} \frac{e^{-\frac{1}{t}}}{t} d t$ and $e^{\frac{1}{x+\xi}} \int_{0}^{x+\xi} \frac{e^{-\frac{1}{t}}}{t} d t$ are functions in Lagrange's sense and that $\sum_{k=0}^{\infty}(-1)^{k} k ! \xi^{k+1}$ is the power-series expansion of the former, this could be considered as a counter-example to theorem 3 . Still, it is doubtful that Lagrange would have accepted these as functions. Certainly, they are not functions according to the compositional conception.

This last example not only shows, like Cauchy's, that Lagrange's notion of function is too restricted; it also shows that his notion of a power-series expansion of a function is too restricted as well.

3.1.3. Lagrange's Proof of the power-series expansion theorem. To claim that in the universe of Lagrange's functions there are no easy counter-example to theorem 3 and that the power-series expansion theorem should be understood as being equivalent to this last theorem is not the same as claiming that Lagrange's proof of this last theorem is sound. Indeed quite the opposite is true: this proof is not only based on premise $[\mathbf{P}]$ - which, however it might be understood, is unproved-and on three other premises like [C.i-iii], that are at best unclarified. It would remain flawed even if these premises were accepted. ${ }^{57}$

Let us consider the two reductio that compose it, in turn.

The first reductio is aiming to exclude fractional exponents. ${ }^{58}$ Lagrange begins by supposing that the generalized power-series expansion of $f(x+\xi)$ has the form (4). According to condition [C.i], the function $f(x)$ would then be irrational, and would thus have "a certain number of different values". ${ }^{59}$ But—as "the replacement of $x$ with $x+\xi$ cannot increase or diminish the number of [...] radicals [in $f(x+\xi)]$ and can no more change their nature, as long as $x$ and $\xi$ are indeterminate quantities" $-f(x+\xi)$ would be irrational too and would have this same number of values. ${ }^{60}$ The different values of $f(x)$ would thus combine with the $|n|$ values of the term $p_{\mu}(x) \xi \frac{m}{n}$, and the expansion would have a greater number of values than $f(x+\xi)$, which, according to condition [C.ii], is impossible.

This argument relies on the admission that any radical has several values. To us, this suggests that Lagrange is working with functions with values in $\mathbb{C}$. Another interpretation is possible, however. For Lagrange's argument to work, it is enough to hold that an equation like $z^{n}-y=0$ (where $n$ is a non-negative integer and $y$ a real quantity) can be satisfied by replacing $z$ with $n$ "imaginary values", ${ }^{6}$ merely understood as expressions of the form ' $a+b \sqrt{-1}$, (where $a$ and $b$ are real quantities, in the sense explained in section 2) to be handled according to the well-known operational rules.

This implicit appeal to imaginary values is unique in Lagrange's treatises, and should thus be understood as a special, local device used in a theory that in fact concerns real quantities. ${ }^{62}$ The crucial assumption, here, is that-when imaginary values for the radicals occurring in $f(x+\xi)$ and in its generalized power expansion are considered-this expansion has to have the same number of values as $f(x+\xi)$. This is an assumption which Lagrange seems to draw, in turn, from the other assumption that the relations that subsist between a function $f(x+\xi)$ and its generalized power expansion, when the variables of the relevant functions vary on real quantities, also subsist when these variable are supposed to take imaginary values: an assumption that remains unjustified, in turn.

The second reductio is aiming to exclude negative exponents. Lagrange begins by supposing that, if a function $f(x+\xi)$ had a generalized power-series expansion that contains a term of the form $p_{\mu}(x) \xi^{-m}$ — where $\mu$ is a non-negative integer and $m$ a positive exponent - then, for $\xi=0$, this term, and thus also $f(x+\xi)$, would become infinite. It would follow that

${ }^{57}$ This seems to have been Galois' opinion too. In a note published in Galois (EMM, pp. 413-421), but not dated, he remarks that in order to prove that the generalized power-series expansion of $f(x+\xi)$ cannot contain fractional or negative powers of $\xi$, Lagrange "produces an argument that collapses on its own" (ibid., p. 413). It is not clear, however, what flaws Galois had in mind.

${ }^{58}$ Grabiner (1990, pp. 98-99) has pointed out a number of flaws relative to this first reductio, some of which do no seem to subsist to us. We do not consider them here.

${ }^{59}$ According to Woodhouse (1803, p. XIX), Lagrange's argument would be circular, since, to prove that "any radical has as many values as there are unities in its exponent", it would be necessary to rely on the Eulerian equality $\cos \theta+\sqrt{-1} \sin \theta=e^{\theta \sqrt{-1}}$ which derives in turn from the expansions of the exponential, the sine and the cosine. This last equality is, however, only useful to exhibit explicitly the radicals of the equation $x^{n}-\zeta=0$ (where $n$ is a natural exponent and $\zeta$ a complex number). The number of them depends, more generally, on the fundamental theorem of algebra, which is the result Lagrange refers to when speaking generally of the theory of equations.

${ }^{60}$ The aforementioned function $\sqrt{x}$ provides an easy example of what happens if $x$ takes a value-namely, the value $x=0$ - for which $f(x+\xi)$ and $f(x)$ do not have the same number of radicals. Another straightforward example is provided by the function $\frac{\sqrt{x-a}}{x}$ for $x=a$. In this case, the generalized power-series expansion of $f(a+\xi)=\frac{\sqrt{\xi}}{a+\xi}$ is $\sum_{k=0}^{\infty}(-)^{k} a^{-1-k} \xi^{\frac{2 k+1}{2}}$.

${ }^{61}$ The term 'imaginary value' was common in Lagrange's time: for an example, see footnote (62), below.

${ }^{62}$ This was a common practice in 18th-century mathematics that Gauss describes quite well in 1811 by observing that "imaginary values" were often treated as an "excrescence [Überbein]" of "real magnitudes": see Gauss (WERKE), vol. 10.1, p. 366 (letter of Gauss to Bessel, December, 18, 1811). The former were merely regarded as forming a sort of analogical extension of the latter to be considered in certain particular circumstances, without investigating the general consequences of their use: see Ferraro (2007), p. 484. 
$f(x)$ would be infinite for any $x$, whereas this is possible only for "particular values of $x$ " ${ }^{63}$ We suggest that Lagrange implicitly assumes, here, that $f(x+\xi)$ becomes infinite if its generalized power-series expansion becomes infinite. So his argument implicitly relies on condition [C.iii], and on the assumption that the generalized power-series expansion of $f(x+\xi)$ converges to this function for $\xi=0$, according to our interpretation of premise $[\mathbf{P}]$.

Two final remarks about Lagrange's proof are in order. The former is that the appeal to condition [C.i] in the first reductio is strictly unnecessary. Suppose a function $f(x+\xi)$ has a generalized power-series expansion that contains both $f(x)$ and a term having more than one value, and that $f(x)$ has as many values as $f(x+\xi)$ (which happens, of course, if they both have only one value). It is then obvious that the number of values in this expansion is greater than the number of values of $f(x+\xi)$. The latter remark is that, in the second reductio, Lagrange implicitly excludes the possibility that a function $f(x+\xi)$ has a generalized power-series expansion containing several terms of the form $p_{\mu}(x) \xi^{-m}$ (distinct from each other for the value of $m$, of course), whose sum is not infinite for $\xi=0$. This seems to be correct, however, since this possibility requires that the values of the coefficients of these terms depend on that of $\xi$, which is unacceptable.

3.2. The Recursive Form of Power-Series Expansions. Lagrange's fundamental proof is entirely based on the powerseries expansion theorem. This proof is performed in fours steps. The first one consists in the proof of this last theorem. In the second step, Lagrange proves that the power-series expansion of any function $f(x+\xi)$ has a recursive form. In the third, he relies on this form for fixing the algorithm of derivative functions. This is what allows him to prove that conditions [FC. $i$ ] holds. Lastly, in the fourth step, Lagrange wonders what makes that, for a particular value $x_{0}$ of $x$, the function $f\left(x_{0}+\xi\right)=f_{x_{0}}(\xi)$ has no power-series expansion. This is what allows him to prove that also condition [FC.ii] holds.

In the present section we deal with the second step (Lagrange 1797, art. 16; 1801 pp. 10-13; 1806 pp. 10-13; 1813, art. I.8). Sections 3.3 and 3.4 will be devoted to the third and the fourth ones, respectively.

This is how Lagrange states, in the Théorie, the result proved in the second step:

$[\ldots]$ if $[\ldots]$ one denotes with $f^{\prime}(x)$ the first derivative function of $f(x)$, with $f^{\prime \prime}(x)$ the first derivative function of $f^{\prime}(x)$, with $f^{\prime \prime \prime}(x)$ the first derivative function of $f^{\prime \prime}(x)$, and so on, one will get [...]

$$
f(x+\xi)=f(x)+f^{\prime}(x) \xi+\frac{f^{\prime \prime}(x)}{2} \xi^{2}+\frac{f^{\prime \prime \prime}(x)}{2.3} \xi^{3}+\frac{f^{\mathrm{IV}}(x)}{2.3 .4} \xi^{4}+\& c .
$$

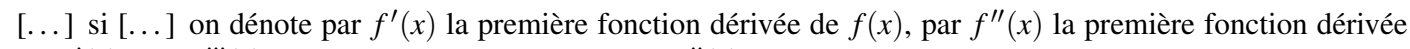
de $f^{\prime}(x)$, par $f^{\prime \prime \prime}(x)$ la première fonction dérivée de $f^{\prime \prime}(x)$, et ainsi de suite, on aura $[\ldots]$

$$
f(x+\xi)=f(x)+f^{\prime}(x) \xi+\frac{f^{\prime \prime}(x)}{2} \xi^{2}+\frac{f^{\prime \prime \prime}(x)}{2.3} \xi^{3}+\frac{f^{\mathrm{IV}}(x)}{2.3 .4} \xi^{4}+\& c .
$$

(Lagrange 1797, p. 14; 1813, p.18; an analogous claim is also made in the Leçons: 1801 pp. 12-13: 1806 pp. 12-13.)

Supposing that $g(x)$ is any function, the term 'première fonction dérivée de $g(x)$ ' is clearly used here to refer to the coefficient of $\xi$ in the power-series expansion of $g(x+\xi)$, whereas the symbol of equality is used to denote the relation between the function $f(x+\xi)$ and its power-series expansion. Lagrange's result can then be stated, more clearly, as follows:

Theorem 4. The power-series expansion of any function $f(x+\xi)$ has the form:

$$
\sum_{k=0}^{\infty} \frac{f^{(k)}(x)}{k !} \xi^{k}
$$

where $f^{(0)}(x)=f(x)$ and, for any positive integer $v, f^{(v)}(x)$ is the coefficient of $\xi$ in the power-series expansion of $f^{(v-1)}(x+\xi)$.

This same result had been already advanced by Lagrange himself in his 1772 memoir on a "new species of calculus" (Lagrange 1772, pp. 186-189; on Lagrange's 1772 memoir, see Grabiner 1990, pp. 31-39; Panza 1992, pp. 569-593). The proof offered in the Théorie and the Leçons is the same as that offered in this memoir. ${ }^{64}$

This proof is not only based on the assumption that any function of a binomial has a unique expansion under the form of a power-series of the second term of such a binomial, as stated by theorem 3 . It also requires that the replacement of such a function with its power-series expansion is licensed independently of any consideration concerning the interval of convergence of the latter. This was a current practice in 18th-century analysis, and Lagrange follows it unwaveringly.

\footnotetext{
${ }^{63}$ The aforementioned function $\frac{1}{x}$ provides an easy example of what happens if $x$ takes a value-namely $x=0$ —for which $f(x)$ becomes infinite. Another easy example is provided by the function $\frac{\sqrt{x}}{x-a}$ for $x=a$. In this case, the generalized power-series expansion of $f(a+\xi)=\frac{\sqrt{a+\xi}}{\xi}$ is $\sum_{k=0}^{\infty}\left(\begin{array}{c}\frac{1}{2} \\ k\end{array}\right) a^{\frac{1}{2}-k} \xi^{k-1}$

${ }^{64}$ An alternative proof of theorem 4 is offered by Poisson: see Poisson (1804-1805); Panza (1992), pp. 736-741.
} 
The basic idea is close in spirit to the solution of a functional equation. It consists in equalizing the power-series expansions of $f((x+\xi)+o)$ and $f(x+(\xi+o))$, supposing that $\xi$ and $o$ are two independent indeterminate increments. ${ }^{65}$

These expansions are respectively:

$$
\sum_{k=0}^{\infty} p_{k}(x+\xi) o^{k} \quad \text { and } \quad \sum_{k=0}^{\infty}\left[p_{k}(x)\right](\xi+o)^{k} .
$$

If in the former, the functions $p_{k}(x+\xi)$ are replaced by their power-series expansions, and in the latter, the powers of the binomial $\xi+o$ are developed, one obtains:

$$
\sum_{k=0}^{\infty}\left[\sum_{h=0}^{\infty} q_{k, h}(x) \xi^{h}\right] o^{k} \quad \text { and } \quad \sum_{k=0}^{\infty}\left[\sum_{h=k}^{\infty}\left(\begin{array}{c}
h \\
k
\end{array}\right) p_{h}(x) \xi^{h-k}\right] o^{k} .
$$

Hence, by equalizing these series and applying twice the method of indeterminate coefficients, so as to equalize first the coefficients of $o^{k}$ in these same series, and then the coefficients of $\xi$ in those of $o^{k}$, one easily gets the equalities:

$$
p_{k+1}(x)=\frac{q_{k, 1}(x)}{k+1} \quad(k=0,1,2, \ldots) .
$$

Since $q_{k, 1}(x)$ is the coefficient of $\xi$ in the power-series expansion of $p_{k}(x+\xi)$ and $p_{0}(x)$ is nothing but $f(x)$, it is enough to denote with ' $f^{(v)}(x)$ ' the coefficient of $\xi$ in the power-series expansion of $f^{(v-1)}(x+\xi)(v=1,2, \ldots)$-by supposing that $f^{(0)}(x)$ be $f(x)$-and to admit that the coefficient of $\xi$ in the power-series expansion of $\frac{f^{(\lambda)}(x+\xi)}{\lambda !}(\lambda=2,3, \ldots)$ is obtained by multiplying by $\frac{1}{\lambda !}$ the coefficient of $\xi$ in the power-series expansion of $f^{(\lambda)}(x+\xi)$, in order to rewrite the equality (9) as follows:

$$
p_{v}(x)=\frac{q_{v-1,1}(x)}{v}=\frac{f^{(v)}(x)}{v !} \quad(v=1,2, \ldots),
$$

as was to be proved.

Once this theorem proved, the meaning of the term 'derivative function [fonction dérivée]' can be fixed in a more precise way, namely as follows:

We shall call the function $f(x)$ 'primitive function' with respect to the functions $f^{\prime}(x), f^{\prime \prime}(x), \& c$. that derive from it, and we shall call these latter functions 'derivative functions' with respect to the former one. Furthermore, we shall term the first derivative function $f^{\prime}(x)$ 'prime function', the second derivative function $f^{\prime \prime}(x)$ 'second function', the third derivative function $f^{\prime \prime \prime}(x)$ 'third function', and so on.

Nous appellerons la fonction $f(x)$, fonction primitive, par rapport aux fonctions $f^{\prime}(x), f^{\prime \prime}(x)$, \&c. qui en dérvent, et nous appellerons celles-ci, fonctions dérivées, par rapport à celle-là. Nous nommerons de plus la première fonction dérivée $f^{\prime}(x)$, fonction prime; la seconde fonction dérivée $f^{\prime \prime}(x)$, fonction seconde; la troisième fonction dérivée $f^{\prime \prime \prime}(x)$, fonction tierce, et ainsi de suite.

(Lagrange 1797 , art. $17 ; 1801$, p. $13 ; 1806$, p. $13 ; 1813$, art. I.9.) ${ }^{66}$

During his proof, Lagrange does not use indices, and writes many relevant equalities only up to the first term of the expansions involved in them, by denoting respectively with ' $p$ ', ' $q$ ', ' $r$ ', 's', \&c., the coefficients of $\xi$, $\xi^{2}, \xi^{3}, \xi^{4}, \& c$. in the power-series expansion of $f(x+\xi)$, and by ' $p$ ', ' $q$ ', ' $r$ ', \&c. the coefficients of $o$ in the power-series expansions of $p(x+o)$, ' $q(x+o)$ ', ' $r(x+o)$ ', \&c. ${ }^{67}$ This fact together with his use of symbols ' $f^{\prime}(x)$ ', ' $f^{\prime \prime}(x)$ ' \&c. suggest the idea that the derivative functions result from the primitive one by reiteratively applying to it an appropriate operator. This idea is however neither explicit in Lagrange's argument, not necessary for his argument to work. The simple identification of $f^{\prime \ldots \prime \prime}(x)$ (or $f^{(v)}(x)$ ) with the coefficient of $\xi$ in the power-series expansion of $f^{\prime \ldots \prime}(x+\xi)$ (or $f^{(v-1)}(x+\xi)$ ), and that of $p^{\prime}, q^{\prime}, r^{\prime}, \& c$. with the coefficient of $\xi$ in the power-series expansion of $p(x+o),{ }^{\prime} q(x+o)$ ', ' $r(x+o)$ ', \&c. are enough.

\footnotetext{
${ }^{65}$ For reasons of clarity, we have permuted $\xi$ and $o$ in Lagrange's proof.

${ }^{66}$ In the Leçons, one finds 'première fonction dérivée, ou fonction dérivée du premier ordre, ou simplement fonction prime' in place of 'fonction prime', and similarly for the second and third derivatives.

${ }^{67}$ Like the symbol ' $p_{h}$ ', also the he symbol ' $q_{k, h}$ ' is introduced by us to simplify Lagrange's notation. Here's how this notation is introduced in the Théorie:

$[\ldots]$ let $f x+f^{\prime} x o+\& c ., p+p^{\prime} o+\& c ., q+q^{\prime} o+\& c ., r+r^{\prime} o+\& c$. be that which the functions $f x, p, q, r, \& c$. become when $x$ is replaced with $x+o[\ldots]$.

[...] soit $f x+f^{\prime} x o+\& c ., p+p^{\prime} o+\& c ., q+q^{\prime} o+\& c ., r+r^{\prime} o+\& c$., ce que deviennent les fonctions $f x, p, q, r, \& c$. en y mettant $x+o$ pour $x$ [...].

(Lagrange 1797, art. 16; 1813, art. I.8.)
} 
3.3. The Algorithm of the Derivatives Functions. We are now ready to consider the third step of Lagrange's fundamental proof.

At the beginning of the Théorie, Lagrange refers to his 1772 memoirs and claims to have there argued that "the theory of expansion of functions" contains the "true principles of differential calculus freed from any consideration of infinitely small or limits" and then to have proved, through this theory, Taylor's theorem, that, he says, had been before only proved through the differential calculus (Lagrange 1797, art. 7; 1813, Introduction, p. 5).

This is only partially true. In this memoir, Lagrange argues that "the differential calculus, considered in all its generality, consists in finding, by simple and easy procedures, the functions [...] derived from the function $f$ " which provides the coefficient of the power-series expansion of $f(x+\xi)$ (Lagrange 1772, p. 187). ${ }^{68}$ But he justifies it by relying on the differential formalism and on the practice of neglecting differentials of higher orders, so as to get Taylor's theorem under then form

$$
f(x+\xi)=\sum_{k=0}^{\infty} \frac{d^{k} f}{d x^{k}} \frac{\xi^{k}}{k !} .
$$

Far from being independent of the differential calculus, this result is basically concerned with it, and is thus essentially different from theorem 4 (Grabiner 1990, p. 37). Hence, Lagrange certainly proves, in his 1772 memoir, the equivalence between the derivative functions entering the power-series expansion of a function $f(x+\xi)$ and the differential quotients $\frac{d^{k} f}{d x^{k}}(k=1,2, \ldots)$. Moreover, his proof undoubtedly provides a grounds for asserting that "the differential calculus [...] consists in finding [...] the functions [...] derived from the function $f$ ". But, this proof depends on essential properties of differentials. In his 1772 memoir, Lagrange is thus still quite far from arguing that "the theory of series expansion of functions" contains the "true principles of differential calculus freed from any consideration of infinitely small or limits".

This is what he does, instead, in the Théorie and in the Leçons through a completely new argument: he first findsrelying neither on infinitesimalist considerations nor on the formalism of calculus - the first derivatives of his elementary functions; then he shows how to compose these derivatives so as to get those of the composed and implicit functions; in this way, he implicitly shows that the algorithm to be applied for getting the function $f^{(v)}(x)$ from the function $f^{(v-1)}(x)$ $(v=1,2, \ldots)$ is the same as that employed for passing from $\frac{d^{v-1} y}{d x^{v-1}}$ to $\frac{d^{v} y}{d x^{v}}$.

The first elementary function that Lagrange considers is $x^{m}$, where $m$ is a rational exponent. A very simple way of finding its first derivative consists in applying the binomial theorem for rational exponents. As this theorem can be easily proved relying neither on infinitesimalist considerations nor on the calculus, such a procedure presents no difficulty. But, it is unnecessarily cumbersome, since we may attain the same ends by relying on this same theorem restricted to integer positive exponents.

If $m=\frac{\mu}{v}$ or $m=-\frac{\mu}{v}$ (where $\mu$ and $v$ are positive integer), it suffices to suppose that

$$
(x+\xi)^{\frac{\mu}{v}}=x^{\frac{\mu}{v}}+A_{1} \xi+\& c . \quad \text { and } \quad(x+\xi)^{-\frac{\mu}{v}}=\frac{1}{(x+\xi)^{\frac{\mu}{v}}}=x^{-\frac{\mu}{v}}+B_{1} \xi+\& c .
$$

and apply this theorem so restricted, and then the method of indeterminate coefficients, to conclude that:

$$
\begin{array}{ccc}
\mu x^{\mu-1}=v x^{\frac{\mu(v-1)}{v}} A_{1} & \text { or } & A_{1}=\frac{\mu}{v} x^{\frac{\mu-v}{v}} \\
v x^{\frac{\mu}{v}} B_{1}+\mu x^{-1}=0 & \text { or } & B_{1}=-\frac{\mu}{v} x^{-\frac{\mu+v}{v}} .
\end{array}
$$

It is thus clear why, in the Théorie (Lagrange 1797, art. 18; 1813, art. I.10), Lagrange merely argues that, insofar as $m$ is rational, "it is easy to prove, either through the simple rules of arithmetic, or through the first operations of algebra", that the first derivative of $x^{m}$ is $m x^{m-1}$.

This result is not strong enough, however, for providing a basis for obtaining the first derivatives of the transcendent elementary functions. For that, the binomial theorem extended to exponents given by any sort of algebraic quantity-or, at least, the part of this theorem concerning the first two terms of the expansion-is necessary. This is certainly the reason why, in the Leçons (Lagrange 1801, pp. 13-17; 1806, p. 16-21), Lagrange finds the coefficient of $\xi$ in the power-series expansion of the function $(x+\xi)^{m}$ through an argument that holds also if $m$ is not rational. This consists in the solution of the functional equation occurring in the Æpinus-Euler proof of the binomial theorem (see p. 15, above).

Supposing that

$$
(1+z)^{m}=1+F(m) z+\& c .
$$

where $F(m)$ is an indeterminate function of $m$, and relying on the equality $(1+z)^{m+n}=(1+z)^{m}(1+z)^{n}$ and the method of indeterminate coefficients, Lagrange gets

$$
F(m+n)=F(m)+F(n),
$$

\footnotetext{
${ }^{68}$ Lagrange write here ' $u$ ' instead of ' $f$ '.
} 
which is just the Æppinus-Euler equation. Next, he remarks that $(1+z)^{m+n}=(1+z)^{(m+\xi)+(n-\xi)}$ and applies again the method of indeterminate coefficients, together with theorem 4, yielding:

$$
\begin{aligned}
F(m+n) & =F(m+\xi)+F(n-\xi) \\
& =F(m)+F(n)+\left[F^{\prime}(m)-F^{\prime}(n)\right] \xi+\& c .
\end{aligned}
$$

He can then compare this equality with the Æppinus-Euler equation and apply once more the method of indeterminate coefficients, to obtain that $F^{\prime}(m)=F^{\prime}(n)$, which shows that $F^{\prime}(m)$ does not depend on $m$, that is, it is constant.

To solve the Epinus-Euler equation it is thus enough to find "the value of the primitive function $F(m)$ based on the derivative function $F^{\prime}(m)=a$ ", where $a$ is an indeterminate constant (Lagrange 1801, p. 131; 1806, p. 19). To do it, Lagrange relies on the condition $F^{(v)}(m)=0(v=2,3, \ldots)$, to get $F(m+\xi)=F(m)+a \xi$, and thus, for $\xi=-m$ : $F(m)=a m+F(0) .{ }^{69}$ Having obtained this last equality, Lagrange compares it with the equality (13), sets $m=0$ and $m=1$, and obtains, once again through the method of indeterminate coefficients, $F(0)=0$ and $a=1$, and thus: $F(m)=m$. It is thus enough to replace this value in the equality (13) and suppose that $z=\frac{\xi}{x}$, to obtain:

$$
(x+\xi)^{m}=x^{m}\left(1+\frac{\xi}{x}\right)^{m}=x^{m}+m x^{m-1} \xi+\& c . \quad \text { and thus } \quad\left(x^{m}\right)^{\prime}=m x^{m-1} .
$$

The possibility of relying on this equality, where $m$ can be supposed to be any algebraic quantity, makes the determination of the derivatives of $a^{x}$ and $\log _{a} x$ very easy.

The determination of the derivatives of trigonometric functions is a bit more elaborate because of the intrinsic geometric nature of these functions (see p. 13, above) ${ }^{70}$ To obtain these derivatives, Lagrange supposes, in the first edition of the Théorie and in the Leçons, that these functions satisfy certain suitable conditions that he does not justify, while in the second edition of the Théorie, he defines them in terms of imaginary exponentials. There is no need to detail Lagrange's arguments on these matters (the references are given at p. 15, above).

On the base of these results, it is then also easy to show how the derivatives of $f(x) \pm g(x), f(x) \cdot g(x)$ and $\frac{f(x)}{g(x)}$ are composed by those of $f(x)$ and $g(x)$. Lagrange's arguments for it (Lagrange 1797, art. 30; 1801 p. 36-38; 1806 pp. 47-49; 1813, art. I.15) are similar in nature to those he relies on for showing how to find the first derivatives of functions whose arguments are other functions and of implicit functions.

The former of these two last arguments runs as follows (Lagrange 1797, art. 31; 1801 p. 38; 1806 p. 49; 1813, art. I.16). Let $g(x)$ be a function of $x$ and $f(g(x))$ be a function of $g(x)$; if $o$ is the increment of $g(x)$ that corresponds to the increment $\xi$ of $x$, then, according to theorem 4 :

$$
\begin{aligned}
o=\sum_{h=1}^{\infty} \frac{1}{h !}\left[g^{(h)}(x)\right] \xi^{h} \quad \text { and } \quad f(g(x)+o) & =f(g(x))+f^{\prime} g(x)(g(x)) o+\& c . \\
& =f(g(x))+g^{\prime}(x) f^{\prime} g(x)(g(x)) \xi+\& c .
\end{aligned}
$$

where $f^{\prime} g(x)(g(x))$ is the coefficient of $o$ in the power-series expansions of $f(g(x)+o)$, i. e. the first derivative of $f(g(x))$ with respect to $g(x)$. Hence, the coefficient of $\xi$ in the power-series expansions of $f(g(x+\xi))-i$. e. the first derivative of $f(g(x))$ with respect to $x$-is $g^{\prime}(x) f^{\prime} g(x)(g(x))$. If one denotes this derivative with ' $f^{\prime} x(g(x))$ ', one obtains, then, the following equality ${ }^{71}$

$$
f^{\prime} x(g(x))=g^{\prime}(x) f^{\prime} g(x)(g(x)),
$$

in conformity with the chain rule for the differential calculus.

Lagrange's argument for showing how to find the derivatives of implicit functions is not much trickier (Lagrange 1797, art. 33; 1801 pp. 39-42; 1806 pp. 50-54; 1813, art. I.17).

Let $F(x, y)=0$ be an equation involving elementary functions of two variables algebraically composed, and $y=f(x)$ a root of it. The function $F(x, f(x))=\varphi(x)$ has then to be null, to the effect that $\varphi^{\prime}(x)=0$ (see footnote 69 , above). To find the first derivative of $f(x)$, it suffices to write this equality in a convenient form.

For this purpose, let $F(p(x), q(x))$ be a function of two variables that are, in turn, functions of a unique variable $x$. The function $F(p(x+\xi), q(x+\xi))$ can then be expanded in a power series of $\xi$. Lagrange takes it for granted that this power series can be obtained by appropriately composing the power-series expansions of $F(p(x+\xi), q(x))$ and $F(p(x), q(x+\xi))$, where $q(x)$ and $p(x)$ are respectively handled as if they were constant. In this way, he gets

$$
F(p(x+\xi), q(x+\xi))=F(p(x), q(x))+\left[\begin{array}{c}
p^{\prime}(x) F^{\prime} p(x)(p(x), q(x))+ \\
q^{\prime}(x) F^{\prime} q(x)(p(x), q(x))
\end{array}\right] \xi+\& c .
$$

\footnotetext{
${ }^{69}$ An easy way to prove that the condition $F^{(v)}(m)=0(v=2,3, \ldots)$ holds is to remark that if $f(x)=a$, then also $f(x+\xi)=a$, for any $\xi$, and thus the equality $f(x+\xi)=\sum_{k=0}^{\infty} \frac{f^{(k)}(x)}{k !} \xi^{k}$ reduces to $0=\sum_{k=1}^{\infty} \frac{f^{(k)}(x)}{k !} \xi^{k}$, from which, according to the method of indeterminate coefficients, it follows that $f^{(k)}(x)=0(k=1,2, \ldots)$.

${ }^{70}$ According to Ovaert (Ovaert (1976, p. 186), Lagrange is forced here to abandon the purely formal level.

${ }^{71}$ The symbols ' $f^{\prime} g(x)(g(x))$ ' and ' $f^{\prime} x(g(x))$ ' are not Lagrange's. His notation is much less explicit.
} 
where $F^{\prime} p(x)(p(x), q(x))$ and $F^{\prime} q(x)(p(x), q(x))$ are, respectively, the first derivatives of $F(p(x), q(x))$ with respect to $p(x)$ $(q(x)$ being handled as if it were constant) and with respect to $q(x)(p(x)$ being handled as if it were constant), $i . e$. the coefficients of $\xi$ and $o$ in the power-series expansions of $F(p(x)+\xi, q(x))$ and of $F(p(x), q(x)+o)$. It follows that, if $p(x)=x$ and $q(x)=y=f(x)$, then

$$
F^{\prime} x(x, f(x))=\varphi^{\prime}(x)=0=F^{\prime x}(x, y)+f^{\prime}(x) F^{\prime} y(x, y),
$$

and thus

$$
f^{\prime}(x)=-\frac{F^{\prime} x(x, y)}{F^{\prime} y(x, y)},
$$

where $F^{\prime} x(x, y)$ and $F^{\prime} y(x, y)$ are, respectively, of course, the first derivatives of $F(x, y)$ with respect to $x$ and $y, i$. $e$. the coefficients of $\xi$ and $o$ in the power-series expansions of $F(x+\xi, y)$ and $F(x, y+o)$, respectively.

All these arguments show that, once theorem 4 and the unicity of the power-series expansion are accepted, the fixation of the algorithm of derivative functions obeys to the compositional conception of function, and depends, essentially, on a number of clever applications of the method of indeterminate coefficients.

3.4. Special Cases. To complete Lagrange's fundamental proof, it is finally necessary to consider the special cases for which the assumption that the generalized power-series expansion of $f(x+\xi)$ contains only integer positive powers of $\xi$ fails, so as to prove that condition [FC.ii] holds.

According to Lagrange's official definition of derivatives, the $v$-th derivative of a function $f(x)$ is the coefficient of $\frac{\xi^{v}}{v !}$ in the power-series expansion of $f(x+\xi)$ or, equivalently, the coefficient of $\xi$ in the power-series expansion of $f^{(v-1)}(x+\xi)$. Hence, strictly speaking, if some terms of the power-series expansion of a function $f(x+\xi)$ are not defined for a particular value of $x$, so that the expansion is not defined either, the function $f(x)$ has no derivatives at all for this value of $x$. On the basis of this definition, there is thus no room for arguing that $f^{(v)}(x)$ is defined for $x=x_{0}$ if and only if the function that results from $f(x)$ by $v$ reiterative applications of the algorithm that leads from it to $f^{\prime}(x)$ is defined for $x=x_{0}$. According to this definition, either all derivatives of a given primitive $f(x)$ are defined for a certain value of $x$, or none. But then how can one account for a case where only a finite number of derivatives of a function $f(x)$ are defined for a certain value of $x$ ?

Lagrange's answer comes from his treatment of the special cases where the generalized power-series expansion of $f(x+\xi)$ is not a power series of $\xi$ (Lagrange 1797, arts. 34-44; 1801 leçon VIII, pp. 52-65; 1806, leçon VIII, pp. 69-87; 1813, ch. V, arts. I.24-I.32.). The main conclusion he reaches is stated in the Théorie as following:

From there, it follows that the expansion $f(x)+\xi f^{\prime}(x)+\frac{\xi^{2}}{2} f^{\prime \prime}(x)+\& c$. can become wrong for a given value of $x$ only insofar as, for this value of $x$, one of the functions $f(x), f^{\prime}(x), f^{\prime \prime}(x)$, \&c. will become infinite as well as all the following ones. Then, if $n$ is the index of the first function that becomes infinite, the relevant expansion will have to contain a term of the form $\xi^{m}$, where $m$ is a number included between $n-1$ and $n$. And if all the functions $f(x), f^{\prime}(x), f^{\prime \prime}(x), \& c$. became infinite for the same value of $x$, the expansion of $f(x+\xi)$ would contain, in this case, some negative powers of $\xi$.

On conclura de là que le que le développement $f(x)+\xi f^{\prime}(x)+\frac{\xi^{2}}{2} f^{\prime \prime}(x)+\& c$. ne peut devenir fautif pour une valeur donnée de $x$, qu'autant qu'une des fonctions $f(x), f^{\prime}(x), f^{\prime \prime}(x)$, \&c. deviendra infinie ainsi que toutes les suivantes pour cette valeur de $x$. Alors si $n$ est l'indice de la première fonction qui devient infinie, le developpement dont il s'agit devra contenir un terme de la forme $\xi^{m}, m$ étant un nombre compris entre $n-1$ et $n$. Et si toutes les fonctions $f(x), f^{\prime}(x), f^{\prime \prime}(x)$, \&c. devenaient infinies pour la même valeur de $x$, le développement de $f(x+\xi)$ contiendrait dans ce cas des puissances négatives de $\xi$.

(Lagrange 1797, art. 42; 1813, art. I.30.) ${ }^{72}$

${ }^{72}$ In the Leçons, this passage is broken up into two. Before, Lagrange writes:

$[\ldots]$ if $n$ is the index of the first function that becomes infinite, the expansion of $f(x+\xi)$ will have to contain a term of the form $\xi^{\alpha}$, where $\alpha$ is a number included between $n-1$ and $n$. Si $n=0$, that is, if the very function $f(x)$ becomes infinite, this expansion will contain some negative powers of $\xi$.

$[\ldots]$ si $n$ est l'indice de la première fonction qui devient infinie, le developpement de $f(x+\xi)$ devra contenir un terme de la forme $\xi^{\alpha}, \alpha$ étant un nombre compris entre $n-1$ and $n$. Si $n=0$, c'est-à-dire, si la fonction $f(x)$ devient elle même infinie, ce developpement contiendra des puissances négatives de $\xi$. (Lagrange 1801 p. $55 ; 1806$ pp. 72-73).

A page later (after having considered the possibility that $f(x+\xi)$ have an expansion including powers of log $\xi$ (see pp. (30-31), below), he adds: One can then conclude in general that the expansion $f(x)+\xi f^{\prime}(x)+\frac{\xi^{2}}{2} f^{\prime \prime}(x)+\& c$. of the function $f(x+\xi)$ can become wrong for a determinate value of $x$ only insofar as one of the functions $f(x), f^{\prime}(x), f^{\prime \prime}(x), \& c$. will become infinite if this value is given to $x$; and that this expansion will only be wrong by starting from the term that becomes infinite.

On peut donc conclure en général que le développement $f(x)+\xi f^{\prime}(x)+\frac{\xi^{2}}{2} f^{\prime \prime}(x)+\& c$. de la fonction $f(x+\xi)$ ne peut devenir fautif pour une valeur déterminée de $x$, qu'autant qu'une des fonctions $f(x), f^{\prime}(x), f^{\prime \prime}(x), \&$ c. deviendra infinie en donnant à $x$ cette valeur; et que ce développement ne sera fautif qu'à commencer du terme qui deviendra infini.

(Lagrange 1801 p. 56; 1806 p. 74.) 
There are three claims in this passage. If the second were taken to apply when $n=0$ (the case where the functions $f(x), f^{\prime}(x), f^{\prime \prime}(x), \& c$. all become infinite), then it would immediately imply the third, at least if the requirement that $m$ lie between $n-1$ and $n$ were understood as the requirement that $n-1<m<n$, and 'negatif' were taken to mean 'less than zero' ${ }^{73}$ Still, the mere consideration of a function like $f(x)=\frac{1}{x^{\alpha}}$, for $\alpha \geq 1$, shows that such an understanding is misleading. This is a case where $n=0$, indeed, but the generalized power-series expansion of $f\left(x_{0}+\xi\right)=f_{x_{0}}(\xi)$, for $x_{0}=0$, reduces to $\frac{1}{\xi \alpha}$, to the effect that $m=-\alpha \leq n-1=-1$.

It seems, then, that the second and third claims apply, in Lagrange's intended understanding, to two distinct cases: the former to the case where $f(x)$ does not become infinite for the relevant value of $x$ but some of its derivatives do; the latter to the case where both $f(x)$ and all its derivatives become infinite for the relevant value of $x$.

This is also confirmed by the way Lagrange argues for his three claims. When reduced to its essential part, his argument runs as follows (Lagrange 1797, art. 41; 1801, pp. 54-55; 1806, pp. 71-73; 1813, art. I.29). ${ }^{74}$

Let $f(x)$ be a function such that the power-series expansion of $f(x+\xi)$ is not defined for $x=x_{0}$. It follows that the generalized power-series expansion of $f\left(x_{0}+\xi\right)$ includes at least a term of the form $A \xi^{m}$, where $A$ does not depend on $\xi$, and $m$ is either a positive fractional or a negative exponent:

$$
f\left(x_{0}+\xi\right)=\ldots+A \xi^{m}+\& c .
$$

The generalized power-series expansion of the first derivative of $f\left(x_{0}+\xi\right)$ with respect to $\xi$ will contain, then, a term of the form $m A \xi^{m-1}$, those of the second derivative, a term of the form $m(m-1) A \xi^{m-2}, \& c$. :

$$
\begin{aligned}
& f^{\prime} \xi\left(x_{0}+\xi\right)=\ldots+m A \xi^{m-1}+\& c . \\
& f^{\prime \prime}\left(x_{0}+\xi\right)=\ldots+m(m-1) A \xi^{m-2}+\& c .
\end{aligned}
$$

But, according to the equality (18), the derivatives of $f(x+\xi)$ with respect to $x$ are the same functions as its derivatives with respect to $\xi$, since both the former and the latter coincide with the derivatives of $f(x+\xi)$ with respect to $x+\xi$. Hence, the values of the derivatives of $f(x)$ for $x=x_{0}$ are obtained by setting $\xi=0$ in the derivatives of $f\left(x_{0}+\xi\right)$ with respect to $\xi$ :

$$
\begin{gathered}
f^{(v)_{x}}(x+\xi)=f^{(v)_{\xi}}(x+\xi)=f^{(v)_{x+\xi}}(x+\xi) \\
\text { and then } \\
{\left[f^{(v)}(x)\right]_{x=x_{0}}=\left[f^{(v)_{\xi}}\left(x_{0}+\xi\right)\right]_{\xi=0},}
\end{gathered}
$$

where $v$ is any positive integer.

From these premises, Lagrange infers that "by setting $\xi=0$, one will conclude that, when $x=x_{0}$, the functions $f(x)$, $f^{\prime}(x), f^{\prime \prime}(x)$, \&c. will respectively contain the terms $A 0^{m}, m A 0^{m-1}, m(m-1) A 0^{m-2}$, \&c." (Lagrange 1797, p. 38; 1801, p. $55 ; 1806$, p. $72 ; 1813$, p. 50$).^{75}$

Lagrange clearly draws this conclusion by setting $\xi=0$ in the equalities (22) and (23), to obtain, in agreement with the second of the equalities (24):

$$
\begin{aligned}
& f\left(x_{0}\right)=\ldots+A 0^{m}+\& c . \\
& f^{\prime}\left(x_{0}\right)=\ldots+m A 0^{m-1}+\& c . \\
& f^{\prime \prime}\left(x_{0}\right)=\ldots+m(m-1) A 0^{m-2}+\& c .
\end{aligned}
$$

However, for this inference to be sound, one must assume that it is enough to set $\xi=0$ in the generalized power-series expansions of the functions $f\left(x_{0}+\xi\right), f^{\prime} \xi\left(x_{0}+\xi\right), f^{\prime \prime} \xi\left(x_{0}+\xi\right), \& c$. to obtain the functions $f\left(x_{0}\right), f^{\prime}\left(x_{0}\right), f^{\prime \prime}\left(x_{0}\right), \& \mathrm{c}$. themselves. Alternatively, one can reach a weaker conclusion by supposing that the generalized power-series expansions of the functions $f\left(x_{0}+\xi\right), f^{\prime} \xi\left(x_{0}+\xi\right), f^{\prime \prime} \xi\left(x_{0}+\xi\right)$, \&c converge to these functions around $\xi=0$. In this latter case, one would merely infer that the values of the functions $f\left(x_{0}\right), f^{\prime}\left(x_{0}\right), f^{\prime \prime}\left(x_{0}\right)$, \&c. are provided by these generalized power-series expansions for $\xi=0$.

However Lagrange might have reasoned, he continues by observing that: if $m$ is negative, all the terms $A 0^{m}, m A 0^{m-1}$, $m(m-1) A 0^{m-2}, \& c$. are infinite, so that the functions $f(x), f^{\prime}(x), f^{\prime \prime}(x)$, \&c. all become infinite for $x=x_{0}$; and if $m$ is positive fractional and $n$ is the positive integer right after $m$, the same happens for the terms $m(m-1) \ldots(m-n+1) A 0^{m-n}$, $m(m-1) \ldots(m-n) A 0^{m-n-1}, \& \mathrm{c}$. and the functions $f^{(n)}(x), f^{(n+1)}(x), \& \mathrm{c}$.

With all this in mind, Lagrange's claims become clearer:

\footnotetext{
${ }^{73}$ We shall return to the meaning Lagrange ascribes to the adjectives 'negatif' and 'positif' in section 5.2.2.

${ }^{74}$ Lagrange presents this argument discursively, without relying on the equalities (22)-(25); we include them to make the presuppositions of the argument clearer.

${ }^{75}$ For consistency with our notation, besides replacing ' $i$ ' by ' $\xi$ ' as usual, we also write ' $x_{0}$ ' instead of ' $a$ '.
} 
- i) A function $f(x+\xi)$ fails to have a power-series expansion for $x=x_{0}-i$. e. the generalized power-series expansion of the corresponding function $f\left(x_{0}+\xi\right)=f_{x_{0}}(\xi)$ contains positive fractional or negative powers of $\xi$-only if one of the functions $f(x), f^{\prime}(x), f^{\prime \prime}(x), \& c$. becomes infinite for $x=x_{0}$ together with all its derivatives.

- ii) A function $f(x+\xi)$ fails to have a power-series expansion for $x=x_{0}$ and the generalized power-series expansion of the corresponding function $f\left(x_{0}+\xi\right)=f_{x_{0}}(\xi)$ contains positive fractional powers and no negative powers of $\xi$ only if one of the functions $f^{\prime}(x), f^{\prime \prime}(x), \& c$. becomes infinite for $x=x_{0}$ together with all its derivatives, and if this is the case and the first of these functions that become infinite is $f^{(n)}(x)$, then the generalized power-series expansion of $f\left(x_{0}+\xi\right)=f_{x_{0}}(\xi)$ contains a power $\xi^{m}$ of $\xi$ such that $n-1<m<n$.

- iii) A function $f(x+\xi)$ fails to have a power-series expansion for $x=x_{0}$ and the generalized power-series expansion of the corresponding function $f\left(x_{0}+\xi\right)=f_{x_{0}}(\xi)$ contains negative powers of $\xi$ if and only if the functions $f(x), f^{\prime}(x), f^{\prime \prime}(x), \& c$. all become infinite for $x=x_{0}$.

Strictly speaking, Lagrange's argument provides a proof for the only-if implication of claim (iii), which is also part of his proof of the power-series expansion theorem. But it does not prove the if implication, though this implication is explicitly stated in the third claim included in the passage quoted above. To justify it, Lagrange could have relied on the following tacit argument: if the generalized power-series expansion of the function $f\left(x_{0}+\xi\right)=f_{x_{0}}(\xi)$ contains no negative powers of $\xi$, then it assumes a finite value for $\xi=0$, and this value is precisely $f\left(x_{0}\right)$, so that $f(x)$ is finite for $x=x_{0}$; hence, if it is not the case that this expansion contains some negative powers of $\xi$, it is no more the case that all the functions $f(x), f^{\prime}(x), f^{\prime \prime}(x)$, \&c. become infinite for $x=x_{0}$; by contraposition, it follows that if the functions $f(x), f^{\prime}(x)$, $f^{\prime \prime}(x)$, \&c. all become infinite for $x=x_{0}$, the generalized power-series expansion of the function $f\left(x_{0}+\xi\right)=f_{x_{0}}(\xi)$ contains negative powers of $\xi$.

The crucial step in this argument amounts to the assumption that the generalized power-series expansion of the function $f\left(x_{0}+\xi\right)=f_{x_{0}}(\xi)$ converges to this function around $\xi=0$, which merely reproduces, in the case where $x=x_{0}$, an assumption that also figures in Lagrange's fundamental proof (see p. 18, above). It is thus quite natural to guess that Lagrange had reasoned correspondingly. But making this assumption amounts to admitting that if the generalized powerseries expansion of the function $f\left(x_{0}+\xi\right)=f_{x_{0}}(\xi)$ contains no negative powers of $\xi$, it can be written in the form

$$
f\left(x_{0}\right)+\sum_{k=1}^{\infty} p_{k}(x) \xi^{\alpha_{k}}
$$

where $\alpha_{k}>0$ for any $k$.

Suppose this is so, and also that the generalized power-series expansion of $f\left(x_{0}+\xi\right)=f_{x_{0}}(\xi)$ contains no negative powers but some positive fractional power of $\xi$, and that $m$ is the smallest exponent of these last powers. This expansion could then be written in the form

$$
f\left(x_{0}\right)+p_{1}(x) \xi+p_{2}(x) \xi^{2}+\ldots+p_{\lfloor m\rfloor}(x) \xi^{\lfloor m\rfloor}+p_{\lfloor m\rfloor+1}(x) \xi^{m}+\& c .,
$$

where $\lfloor m\rfloor$ is the integer right before $m$. But then, reasoning as above, according to the second of the equalities (24), one would obtain

$$
\begin{aligned}
& f^{\prime}\left(x_{0}\right)=p_{1}(x) \\
& f^{\prime \prime}\left(x_{0}\right)=2 p_{2}(x) \\
& \ldots \\
& f^{(\lfloor m\rfloor)}\left(x_{0}\right)=\lfloor m\rfloor ! p_{\lfloor m\rfloor}(x) \\
& f^{(\lceil m\rceil)}\left(x_{0}\right)=m\lfloor m\rfloor ! p_{\lfloor m\rfloor+1}(x) 0^{m-\lceil m\rceil},
\end{aligned}
$$

where $\lceil m\rceil$ is the integer right after $m$, and $m-\lceil m\rceil$ is accordingly a negative exponent. The first derivative of $f(x)$ to become infinite for $x=x_{0}$ would then be $f^{(\lceil m\rceil)}(x)$, so that $n=\lceil m\rceil$ and $n-1<m<m+1$.

This is likely to be the tacit argument Lagrange relied on to justify the second part of claim (ii), which, strictly speaking, does not follows from his explicit argument. By arguing that, in the case at issue, the generalized power-series expansion of $f\left(x_{0}+\xi\right)=f_{x_{0}}(\xi)$ contains a power $\xi^{m}$ of $\xi$ such that $n-1<m<n$, Lagrange would then imply that this power is the smallest of the fractional powers in the expansion.

The difficulties to which Lagrange's arguments (both the explicit and the tacit ones) are subject are obvious. We only point out that they are all based on the identification of the derivatives of a function $f(x)$ for $x=x_{0}$ with the values that can be determined through the application of an appropriate linear algorithms - stemming from the consideration of the power-series expansion of the function $f(x+\xi)$ where $x$ and $\xi$ remain indeterminate- to the equality

$$
f\left(x_{0}+\xi\right)=f\left(x_{0}\right)+\sum_{k=1}^{\infty} p_{k}(x) \xi^{\alpha_{k}} .
$$

This, then, is the implicit definition of these derivatives at issue in Lagrange's theory. It is on the basis of this implicit definition that Lagrange claims to have established his result, namely that: $i$ ) the $v$-th derivative of a function $f(x)$, such 
that $f(x+\xi)$ has no power-series expansion for $x=x_{0}$, is defined for this same value of $x$ if and only if $f\left(x_{0}\right)$ is not infinite and the smallest fractional exponent of the powers of $\xi$ occurring in the generalized power-series expansion of $f\left(x_{0}+\xi\right)=f_{x_{0}}(\xi)$ is greater than $\left.v ; i i\right)$ in this case, the value of this derivative for $x=x_{0}$ is the coefficient of $\frac{\xi^{v}}{v !}$ in this generalized power-series expansion.

As an example, take the function ${ }^{76} f(x)=a x-x^{2}+a\left(x^{2}-a^{2}\right) \sqrt{x^{2}-a^{2}}$. The associated function $f(a+\xi)=f_{a}(\xi)$ is $-a \xi-\xi^{2}+a \xi \sqrt{\xi}(2 a+\xi) \sqrt{2 a+\xi}$, whose generalized power-series expansion is:

$$
-a \xi+2 a^{2} \sqrt{2 a} \xi \sqrt{\xi}-\xi^{2}+\frac{3}{2} a \sqrt{2 a} \xi^{2} \sqrt{\xi}+\frac{3}{16} \sqrt{2 a} \xi^{3} \sqrt{\xi}+\& c .
$$

If follows that

$$
f(a)=0 \text { and } f^{\prime}(a)=-a,
$$

whereas the other derivatives are not defined for $x=a$.

In the Leçons (Lagrange 1801 p. 52 and 55-57; 1806 pp. 69-70 and 73-75), Lagrange also considers the possibility that a function $f\left(x_{0}+\xi\right)=f_{x_{0}}(\xi)$ has an expansion including terms with a factor of the form $\xi^{\lambda}(\log \xi)^{\mu}$, where $\lambda$ and $\mu$ are rational positive or negative exponents. ${ }^{77}$

A simple case is given by the function $f(x)=x \log x$, whose associated function $f\left(x_{0}+\xi\right)=f_{x_{0}}(\xi)$, for $x_{0}=0$, is $\xi \log \xi$. In this case, the relevant value $x_{0}$ belongs to the domain of definition of $f(x+\xi)$ for any non-null value of $\xi$, but not to the domain of definition of $f(x)$ (see p. 20, above). Lagrange begins by proving that this is always the case when the exponent of $\log \xi$ is positive (Lagrange 1801 p. $52 ; 1806$ pp. 69-70). ${ }^{78}$

To this purpose, he relies on an argument analogous to the one he uses for negative powers of $\xi$ : if the generalized power-expansion of $f\left(x_{0}+\xi\right)=f_{x_{0}}(\xi)$ includes a term with a positive power of $\log \xi$ as factor, then this expansion becomes infinite for $\xi=0$ (since $\log \xi=-\infty$ for $\xi=0$ ); the same happens, then, for $f\left(x_{0}\right)$.

He then remarks (Lagrange 1801 pp. 55 and 27; 1806, pp. 73 and 34-35), more generally, the the case of expansions including powers of $\log \xi$ reduces to that of expansions including fractional powers of $\xi$, since, for any base $a$,

$$
\log _{a} z=\frac{r}{\log a}\left(z^{\frac{1}{r}}-1\right)
$$

provided $r$ be "infinitely large". Strangely enough, this appeal to an infinitesimalist argument does not seem detrimental to him. He probably felt the argument was local enough for its infinitesimalist character to be harmless. The fact remains that it is only by appealing to this reduction that he could have justified that the case where $f(x+\xi)$ has an expansion including a power of $\log \xi$ is not mentioned at all in the proof of the power-series expansion theorem, nor, more generally, in the Théorie. Furthermore, for the appeal to equality (32) in this proof to involve no circularity, this last equality should be obtained though an argument different from the one Lagrange uses to obtain it (Lagrange 1801 pp. 24-27; 1806, pp. 3134). This last argument is based, indeed, on the power-series expansion of $\log _{a}(x+\xi)$, which is got, in turn, by relying on the power-series expansion theorem itself. ${ }^{79}$

In any case, Lagrange continues by observing that in the case where the expansion of $f\left(x_{0}+\xi\right)=f_{x_{0}}(\xi)$ includes terms of the form $\xi^{\lambda}(\log \xi)^{\mu}$, an argument analogous to the one used in the case where this expansion contains fractional powers of $\xi$ also applies. In this case, Lagrange says, the derivatives $f^{\prime}(x+\xi), f^{\prime \prime}(x+\xi)$, \&c., for $x=x_{0}$, respectively contain, in tun, terms of the forms $\xi^{\lambda-1}(\log \xi)^{\mu}$ and $\xi^{\lambda-1}(\log \xi)^{\mu-1}$, and of the forms $\xi^{\lambda-2}(\log \xi)^{\mu}, \xi^{\lambda-2}(\log \xi)^{\mu-1}$ and $\xi^{\lambda-2}(\log \xi)^{\mu-2}$, \&c., which, for $\xi=0$, become either null or infinite, depending on whether the exponent of $\xi$ is positive or negative, whatever the exponent of $\log \xi$ might be.

Lagrange's treatment here is rough, but his point is clear: if the expansion of $f\left(x_{0}+\xi\right)=f_{x_{0}}(\xi)$ includes terms of the form $\xi^{\lambda}(\log \xi)^{\mu}$, the expansions of the functions $f^{\prime} \xi\left(x_{0}+\xi\right), f^{\prime \prime} \xi\left(x_{0}+\xi\right)$, \&c., will contain terms like the foregoing, so that the derivatives $f^{\prime}(x), f^{\prime \prime}(x), \& c$., evaluated at $x=x_{0}$, or rather their expansions, will contain terms which become either null or infinite, depending on whether the exponent of $\xi$ is positive or negative. Lagrange also seems to imply that, if the exponent of $\xi$ is 0 , these terms become either null or infinite depending on whether the exponent of $\log \xi$ is negative or positive.

This argument non only poses the same problems as the argument used in the case where the expansion of $f\left(x_{0}+\xi\right)=$ $f_{x_{0}}(\xi)$ contains fractional powers of $\xi$. It also requires the assumption that certain functions vanish, and are then defined, for a particular value of their variable, even if certain terms, factors, or denominators including in them become infinite for

\footnotetext{
${ }^{76}$ This example results from a slight adjustment of an example proposed by Lagrange (1801 p. 56-57; 1806, pp. 74-75).

${ }^{77}$ Though Lagrange does not say so, it is clear that, because of the equality $\log _{a} z=\frac{\log z}{\log a}$, this case encompasses any analogous case concerning logarithms with any base.

${ }^{78}$ Lagrange considers only the case where the factor $\xi^{\lambda}$ in $\xi^{\lambda}(\log \xi)^{\mu}$ is unitary, i. e. $\lambda=0$, but it is clear that the same applies to any value of $\lambda$.

${ }^{79}$ In the Théorie and in the Leçons Lagrange presents two alternative deductions of the power-series expansion of the logarithm. In the first one (Lagrange 1797, art. 21; 1801 pp. 23-24; 1806 pp. 28-3; 1813, art. I.13), he explicitly appeals to the equality (5), which is clearly deduced from the power-series expansion theorem. In the second one (Lagrange 1797, art. 23; 1813, art. I.19), he appeals to the binomial theorem for any real exponent, which is proved in turn by appealing to this same equality: see pp. $25-26$, above.
} 
this value. For example, assuming that, for $\xi=0, \xi^{\lambda}(\log \xi)^{\mu}$ vanishes when $\lambda$ is positive, whatever $\mu$ might be, amounts to assuming that $0( \pm \infty)=\frac{0}{ \pm \infty}=0$. In modern parlance, this amounts to assuming that a function can be extended continuously to a value of its variable where strictly speaking it is not defined. As we have already observed (see p. 20 , above), this is tantamount to replacing this function by one defined piecewise, which seems against Lagrange's notion of function.

To clarify this matter, consider the only example offered by Lagrange (Lagrange 1801 p. 57; Lagrange 1806 p. 75). Let $f(x)$ be $\sqrt{x}+(x-a)^{2} \log (x-a)$. It follows that:

$$
\begin{array}{rlrl}
f(x+\xi) & =\sqrt{x}+(x-a)^{2} \log (x-a)+\left[\frac{1}{2 \sqrt{x}}+(x-a)(2 \log (x-a)+1)\right] \xi+ \\
& = & +\left[-\frac{1}{4 x \sqrt{x}}+2 \log (x-a)+3\right] \frac{\xi^{2}}{2}+\left[\frac{3}{8 x^{2} \sqrt{x}}+\frac{2}{x-a}\right] \frac{\xi^{3}}{3 !}+\& c .
\end{array}
$$

According to Lagrange, all derivatives of this function from $f^{\prime \prime}(x)$ become infinite for $x=0$, so that the expansion of $f(a+\xi)=f_{a}(\xi)$ contains the term $\xi^{2} \log \xi$. Since $f(a+\xi)=f_{a}(\xi)=\sqrt{a+\xi}+\xi^{2} \log \xi$, this expansion is obtained by expanding $\sqrt{a+\xi}$ in a power-series, according to the binomial theorem for positive fractional exponents, so as to get

$$
\sqrt{a+\xi}+\xi^{2} \log \xi=\sqrt{a}+\frac{1}{2 \sqrt{a}} \xi-\left[\frac{1}{8 a \sqrt{a}}-\log \xi\right] \xi^{2}+\frac{1}{16 a^{2} \sqrt{a}} \xi^{3}+\& c .
$$

It seems then that Lagrange take the functions $(x-a)^{2} \log (x-a)$ and $(x-a)(2 \log (x-a)+1)$ to be defined for $x=a$, and namely to vanish. Had he denied this, he could not have taken the coefficient of $\xi$ in the expansion (34) to be the first derivative of $\sqrt{x}+(x-a)^{2} \log (x-a)$ for $x=a$, and argued that the power-series expansion of the associated function $f(x+\xi)$ is not defined only from its third term. He would then have been forced to go against his whole analysis of the special cases where a function $f(x+\xi)$ does not admit a power-series expansion.

Going over to fractional powers according to equality (30) might solve the problem, for then:

$$
\begin{aligned}
f(x+\xi)= & \sqrt{x+\xi}+(x+\xi-a)^{2} \log (x+\xi-a)= \\
= & \sqrt{x+\xi}+r(x+\xi-a)^{2}\left((x+\xi-a)^{\frac{1}{r}}-1\right)= \\
= & \sqrt{x}+r(x-a)^{2}\left((x-a)^{\frac{1}{r}}-1\right)+ \\
& \quad+\left[\frac{1}{2 \sqrt{x}}+(x-a)\left((x-a)^{\frac{1}{r}}(2 r+1)-2 r\right)\right] \xi+ \\
& \quad+\left[\frac{1}{4 x \sqrt{x}}-2 r+(x-a)^{\frac{1}{r}}\left(2 r+3+\frac{1}{r}\right)\right] \frac{\xi^{2}}{2}+\& c .
\end{aligned}
$$

and

$$
\begin{aligned}
f(a+\xi)=f_{a}(\xi) & =\sqrt{a+\xi}+\xi^{2} \log \xi=\sqrt{a+\xi}+\xi^{2} r\left(\xi^{\frac{1}{r}}-1\right)= \\
& =\sqrt{a}+\frac{1}{2 \sqrt{a}} \xi-\left[\frac{1}{8 a \sqrt{a}}+r\right] \xi^{2}+r \xi^{2+\frac{1}{r}}+\frac{1}{16 a^{2} \sqrt{a}} \xi^{3}+\& c .
\end{aligned}
$$

(where $r$ is infinitely large). But, for the values of $f(a)$ and $f^{\prime}(a)$ calculated using the expansions (35) to be equal to those calculated using the expansions (36), and for the value of $f^{\prime \prime}(x)$ calculated using the former of these expansions to become infinite for $x=a$, one has to assume that

$$
\begin{aligned}
{\left[r(x-a)^{2}\left((x-a)^{\frac{1}{r}}-1\right)\right]_{x=a} } & =\left[(x-a)\left((x-a)^{\frac{1}{r}}(2 r+1)-2 r\right)\right]_{x=a} \\
& =\quad\left[(x-a)^{\frac{1}{r}}\left(2 r+3+\frac{1}{r}\right)\right]_{x=a}=0,
\end{aligned}
$$

which is an inescapably infinitesimalist assumption.

It seems then that, once more, Lagrange's mathematical practice departs here from his ideal of purity of method, or, more precisely, from his very notion of function. ${ }^{80}$

\section{Lagrange's Reformulation of the Calculus: Some Examples}

Lagrange's fundamental proof could not have been a sufficient basis for him to argue that his theory of analytical function could (or should) supplant the differential calculus in all its extension and for all its applications. Many of the results forming the corpus of the latter, together with its several applications both geometric and mechanical, had been obtained using arguments based on infinitesimalist interpretations of it, namely on the interpretation of a differential as an infinitely small quantity, or of an integral as an infinite sum of infinitely small quantities. If Lagrange had simply proposed the

\footnotetext{
${ }^{80}$ We should add that Lagrange takes the opportunity of his treatment of the particular values of $x$ for which a function $f(x+\xi)$ fails in having a power-series expansion, for considering the cases where the equality (21) leads to an expression of the derivative of an implicit function that, for a certain value of the relevant variable, reduces to $\frac{0}{0}$. Needless to say, he shows how to deal with this difficulty through l'Hôpital's rule, which he proves in the context of his theory (Lagrange 1797, arts. 36 and 39; 1801 pp. 58-59 and 61-62; 1806 pp. 78-80 and 81-82; 1813, arts. I.26 and I.28).
} 
replacement of the differential calculus by his theory of derivative functions, by appealing to something like an appropriate translation rule for transforming statements using the language of the former in new statements using the language of the latter, he would have implicitly admitted the validity of these arguments.

The theory of derivative functions could thus not be expected to supplant the differential calculus unless the whole corpus of such a calculus might be reformulate within the context of this theory, and the application of its algorithm (understood as the algorithm of derivative function) to the solution of the geometric and mechanical problems might be justified within this same theory. Most of both the Théorie and the Leçons is devoted to this. Of course, Lagrange does not pursue this task in extenso, for doing so would have required much more than a treatise. He confines himself to some important examples. In sections 4.1-4.5, we shall consider some of these examples from the pure part of Lagrange's theory. In section 5.1, we consider other examples concerned with geometric and mechanical applications.

4.1. Partial Derivatives. The first example concerns partial derivatives. Lagrange's interpretation of the calculus is particularly suitable for filling the gap between the differential calculus and the calculus of partial differentials. This is emphasized by Lagrange himself in the Leçons, where he insists on the unity and appropriate organization of mathematics:

$[\ldots]$ the calculus of derivative functions of only one variable naturally leads to the calculus of derivative functions of several variables, which [...] is nothing but a generalization of the former and depends on the same principles. If the inventors of the differential calculus had firstly regarded it as the calculus of derivative functions, they would have naturally and immediately been led to the calculus of derivative functions of several variables, and an half century would not have passed between the discovery of the differential calculus strictly speaking and that of the partial differential calculus, which corresponds to the calculus of derivative functions of several variables. Furthermore, rather than considering this latter calculus as a new calculus, one would have regarded it as a new application, or better as an extension, of the differential calculus, and one would have, from the very beginning, encompassed the different branches of this same calculus that have been for a long time separated and, as it were, isolated, under the same point of view and the same denomination.

[...] le calcul des fonctions dérivées relatives à une seule variable, conduit naturellement à celui des fonctions dérivées relatives à différentes variables, lequel n'est [...] qu'une généralisation du premier, et dépend des mêmes principes. Si les inventeurs du Calcul différentiel l'avaient regardé d'abord comme le calcul des fonctions dérivées, ils auraient été conduit naturellement et immédiatement au calcul des fonctions dérivées relatives à plusieurs variables; et il ne serait pas passé un demi-siècle entre la découverte du calcul différentiel proprement dit, et celle du calcul aux différences partielles, qui répond au calcul des fonctions dérivées relatives à différentes variables. A plus forte raison, au lieu d'envisager ce dernier comme un nouveau calcul, on l'aurait seulement regardé comme une nouvelle application ou plutôt comme une extension du calcul différentiel, et l'on aurait, dès le commencement, embrassé sous un même point de vue et sous une même dénomination, les différentes branches du même calcul, qui ont été longtemps séparées et comme isolées.

(Lagrange 1801, p. 264; 1806, pp. 327-328.)

Lagrange's basic idea is that the power-series expansion of a function of several variables - and then its total derivativescan be obtained step by step: one begins by considering this function as a function of only one of these variables, and expands it in a power series by dealing with the other ones as if they were constants, then expands the derivative functions so obtained as functions of one of the other variables, and so on.

If $f(x, y)$ is a function of two independent variables (Lagrange 1797, arts. 85-86; 1801, pp. 264-268; 1806, pp. 328-332; 1813, arts. I.73-I.74), this procedure gives first:

$$
f(x+\xi, y)=\sum_{k=0}^{\infty} f^{(k)_{x}}(x, y) \frac{\xi^{k}}{k !}
$$

and then

$$
f(x+\xi, y+o)=\sum_{k=0}^{\infty} f^{(k)_{x}}(x, y+o) \frac{\xi^{k}}{k !}=\sum_{k=0}^{\infty} \sum_{h=0}^{\infty} f^{(k)_{x}(h)_{y}}(x, y) \frac{o^{h}}{h !} \frac{\xi^{k}}{k !}
$$

(where the indices appended to ' $(k)$ ' and ' $(h)$ ' specify the variables with respect to which the derivatives are taken).

The same result is obtained by firstly expanding $f(x, y+o)$ and then expanding the derivatives $f^{(h)_{y}}(x+\xi, y)$. Hence the method of indeterminate coefficients leads to the equality

$$
f^{(k)_{x}(h)_{y}}(x, y)=f^{(h)_{y}(k)_{x}}(x, y)
$$


for any pair of non-negative integers $k$ and $h .^{81}$ It follows that the "operations" of derivations with respect to different variables are "absolutely independent of each other" (Lagrange 1797, art. 86; 1801, p. 268; 1806, pp. 331-332; 1813, art. I.74), which is the fundamental principle of partial derivation.

4.2. Singular Primitive Equations. The second example concerns singular solutions of derivative equations (Lagrange 1797, arts. 72-76; 1801, lectures XV-XVIII; 1806, lectures XIV-XVII; 1813, art. I.58-63; we limit ourselves to the general theory of singular solutions as it is formulated in lectures XV and XIV of the first and the second edition of the Leçons, respectively; on this topic, see also Fraser 1987, pp. 45-49). ${ }^{82}$

To begin with (Lagrange 1801, pp. 162-165; 1806, pp. 178-184), let $f\left(x, y, y^{\prime}\right)=0$ be a derivative equation of the first order, where $y$ is a function of $x$, and $y^{\prime}$ is its first derivative. Lagrange conceives this equation as resulting from the elimination of the arbitrary constant $a$ between two other equations $F(x, y, a)=0$ and $F^{\prime}(x, y, a)=0$, where $F^{\prime}(x, y, a)$ is the total derivative of $F(x, y, a)$, that is, the coefficient of $\xi$ in the power-series expansion of $F(x+\xi, y(x+\xi), a)$, and $F(x, y, a)=0$ is the equation to be found that counts as the complete primitive, or the general solution of $f\left(x, y, y^{\prime}\right)=0 .{ }^{83}$

It is easy to see that this last equation would also be obtained from the same two equations $F(x, y, a)=0$ and $F^{\prime}(x, y, a)=0$ if $a$ were taken to be a function of $x$ such that

$$
F^{\prime} a(x, y, a) a^{\prime}=0
$$

(where the subscript ' $a$ ' specifies that the derivatives is taken with respect to $a$ ). So, if $g(x)$ is a function of $x$ such that this last condition is met for $a=g(x)$, the equation $F(x, y, g(x))=0$ is a solution of $f\left(x, y, y^{\prime}\right)=0$ not contained in its general solution. Lagrange call it 'singular primitive equation'. As from the condition $a^{\prime}=0$ it simply follows that $a$ is constant (see the previous footnote 69), this singular primitive equation is the equation in $y$ and $x$ which results from

$$
\left\{\begin{array}{l}
F(x, y, a)=0 \\
F^{\prime} a(x, y, a)=0
\end{array}\right.
$$

provided its solution $a=\psi(x, y)$ is not constant. It is thus clear that a singular primitive equation of a derivative equation of first order contains no arbitrary constant.

A simple example is the following (Lagrange 1801, p. 164; 1806, p. 182). Let $f\left(x, y, y^{\prime}\right)=y^{\prime} \sqrt{x^{2}+y^{2}-b}-y y^{\prime}-x=0$ the derivative equation to be solved. Its general solution is $F(x, y, a)=x^{2}-2 a y-a^{2}-b=0$. If $a$ is taken to be a function of $x$, one gets $F^{\prime a}(x, y, a)=-2 y-2 a$, and the system (42) becomes

$$
\left\{\begin{array}{l}
x^{2}-2 a y-a^{2}-b=0 \\
-2 y-2 a=0
\end{array}\right.
$$

Hence $a=-y$, and $x^{2}+y^{2}-b=0$ is then the singular primitive equation.

A similar argument applies to derivative equations of higher orders (Lagrange 1801, p. 165-166; Lagrange 1806, pp. 184-185). Supposing that $f\left(x, y, y^{\prime}, \ldots y^{(n)}\right)=0$ is such an equation and $F\left(x, y, y^{\prime} \ldots, y^{(n-1)}, a\right)=0$ one of its primitives of order $n-1$, its singular primitive is the equation in $x, y, y^{\prime}, \ldots, y^{(n-1)}$ which results from

$$
\left\{\begin{array}{l}
F\left(x, y, y^{\prime}, \ldots, y^{(n-1)}, a\right)=0 \\
F^{\prime a}\left(x, y, y^{\prime} \ldots, y^{(n-1)}, a\right)=0,
\end{array}\right.
$$

provided its solution $a=\psi(x, y)$ is not constant. Lagrange proves that this singular primitive is unique: for any given derivative equation of order $n$, condition (44) supplies the same singular primitive, whatever primitive equation of order $n-1$ of this derivative equation is considered (Lagrange 1801 , p. 168-169; 1806, pp. 186-190). ${ }^{84}$

He also establishes a sufficient and necessary condition for the existence of such a singular primitive (Lagrange 1801, pp. 173-174; 1806, pp. 195-196). If $a=\Phi\left(x, y, y^{\prime}, \ldots, y^{(n-1)}\right)$ is a root of $F\left(x, y, y^{\prime}, \ldots, y^{(n-1)}, a\right)=0$,

$$
F\left(x, y, y^{\prime}, \ldots, y^{(n-1)}, \Phi\left(x, y, y^{\prime}, \ldots, y^{(n-1)}\right)\right)=0
$$

\footnotetext{
${ }^{81}$ Our notation is different from Lagrange's. He uses two different conventions in the Théorie and in the Leçons. In the former, he denotes the derivatives of a function of two variables with respect to the first variable by ' $f^{\prime \prime \ldots}$ ', and those with respect to the second by ' $f_{1 \prime}$,..; in the latter, he denotes the derivatives with respect to the first variable with ' $f^{\prime \prime} \ldots$,', and those with respect to the second with ' $f$,'...'. This makes difficult for him to notationally distinguish the two functions we have denoted ' $f^{(k)_{x}(h)_{y}}(x, y)$ ' and ' $f^{(h)_{x}(k)_{y}}(x, y)$ ', respectively, for indeterminate indices $k$ and $h$. This is probably why he writes no equality like (40).

${ }^{82}$ Lagrange's derivative and partial derivative equations correspond of course, in the language of his theory, to differential and partial differential equations in the language of differential calculus.

${ }^{83}$ Note that, according to Lagrange's definition of derivative functions, to claim that a function $g(x)$ is the primitive of a function $f(x)$ is the same as claiming that this latter function is such that $g(x+\xi)=g(x)+\xi f(x)+\sum_{k=2}^{\infty} p_{k}(x) \xi^{\alpha_{k}}$ (where $\alpha_{k}$ are rational exponents greater that 1). Lagrange should then provide a proof of existence and uniqueness (except for additive constants) of the primitive of any function. But he offers no such proof.

${ }^{84}$ This proof also suggests a procedure for finding the singular primitive of a derivative equation of order $n$ starting from its complete primitive equation; see Lagrange (1801) p. 169-173; and (1806), pp. 190-195.
} 
is an identical equation. It follows that if the variables $x, y, y^{\prime}, \ldots, y^{(n-1)}$ are taken to be independent of each of other, all the derivatives of the left-hand side of this equation taken with respect to each one of these variables are null. Writing ' $F$ ' and ' $\Phi$ ' for ' $F\left(x, y, y^{\prime}, \ldots, y^{(n-1)}, a\right)$ ' and ' $\Phi\left(x, y, y^{\prime}, \ldots, y^{(n-1)}\right)$ ', one obtains:

$$
\Phi^{\prime x}=-\frac{F^{\prime x}}{F^{\prime} a} \quad ; \quad \Phi^{\prime y}=-\frac{F^{\prime y}}{F^{\prime} a} \quad ; \quad \Phi^{\prime y^{\prime}}=-\frac{F^{\prime y^{\prime}}}{F^{\prime a}} \quad \ldots \quad \Phi^{\prime y^{(n-1)}}=-\frac{F^{y^{(n-1)}}}{F^{\prime a}} .
$$

But as we have just seen, a necessary condition for an equation $f\left(x, y, y^{\prime}, \ldots y^{(n)}\right)=0$ to have a singular primitive is that $F^{\prime} a=0$. The derivative equation $f\left(x, y, y^{\prime}, \ldots y^{(n)}\right)=0$ then has a singular primitive only if

$$
\Phi^{\prime x}=\Phi^{\prime y}=\Phi^{\prime y^{\prime}}=\ldots=\Phi^{y^{(n-1)}}= \pm \infty .
$$

To obtain a sufficient and necessary condition, it is then sufficient to add the requirement that $\Phi$ be not a constant. ${ }^{85}$

4.3. Exact Derivatives. The third example concerns the conditions to be met for a certain function of several variables to be an exact derivative of another function (Lagrange 1801, lecture XIV; 1806, pp 401-409).

Let $\Psi$ be a function of any number of independent variables $x, y, z, \ldots$ and of their derivatives up to whatever order $m$. To begin with the simplest case, let us suppose that this function is linear with respect to one of these variables, say $z$, and to its derivatives, so that: $\Psi=\sum_{k=0}^{m} \Phi_{k} z^{(k)}$, where $\Phi_{k}$ are functions of $x, y, \ldots$ and of their derivatives up to order $m$ (but not of $z$ and its derivatives). As, for any pair of non-negative integers $k$ and $h$,

$$
\left[\Phi_{k}^{(h)} z^{(k-h-1)}\right]^{\prime}=\Phi_{k}^{(h+1)} z^{(k-h-1)}+\Phi_{k}^{(h)} z^{(k-h)},
$$

where the derivatives are total, it is easy to obtain, by reiterated substitutions,

$$
\Phi_{k} z^{(k)}=(-1)^{k} \Phi_{k}^{(k)} z+\sum_{h=0}^{k-1}(-1)^{h}\left[\Phi_{k}^{(h)} z^{(k-h-1)}\right]^{\prime},
$$

and thus:

$$
\Psi=\sum_{k=0}^{m} \Phi_{k} z^{(k)}=\sum_{k=0}^{m}(-1)^{k} \Phi_{k}^{(k)} z+\sum_{k=0}^{m} \sum_{h=0}^{k-1}(-1)^{h}\left[\Phi_{k}^{(h)} z^{(k-h-1)}\right]^{\prime}
$$

(by supposing, of course, that $\left.\sum_{h=0}^{-1}(-1)^{h}\left[\Phi_{0}^{(h)} z^{(-h-1)}\right]^{\prime}=0\right)$.

Hence, $\Psi$ is an exact derivative if and only if $\sum_{k=0}^{m}(-1)^{k} \Phi_{k}^{(k)} z$ is itself an exact derivative, or vanishes. But $\sum_{k=0}^{m}(-)^{k} \Phi_{k}^{(k)} z$ can be an exact derivative only if $z$ is linked to $x, y, \ldots$ through an appropriate relation, which is impossible, since $x, y, z, \ldots$ are supposed to be independent variables. It follows that $\Psi$ is an exact derivative if and only if

$$
\sum_{k=0}^{m}(-)^{k} \Phi_{k}^{(k)}=0
$$

This being proved, Lagrange reduces the general case to such a simpler one.

Let $y$ be a function of $x, V$ and $U$ two functions of $x, y$ and the derivatives of $y$ up to whatever orders $m$ and $m-1$, respectively. Suppose that $V=U^{\prime}$, whatever function of $x, y$ might be. If $z$ is also a function of $x$ and $V_{y \mid y+z}$ and $U_{y \mid y+z}$ are the functions which result from $V$ and $U$ by replacing $y$ with by $y+z$ (and hence $y^{\prime}, \ldots, y^{(m)}$ with $y^{\prime}+z^{\prime}, \ldots, y^{(m)}+z^{(m)}$, respectively), it will follow that $V_{y \mid y+z}=U_{y \mid y+z}^{\prime}$.

\footnotetext{
${ }^{85}$ In Cauchy (1822, pp. 278-279), after having presented the examples mentioned at p. 20, Cauchy claims that his previous considerations suggest that certain results "established by means of series" are faulty. To offers an example, he considers the differential equation $d y=[1+(y-x) \log (y-x)] d x$, and claims that the equation $y=x$ is included in its general solution $\log (y-x)=a e^{x}$, though the differential ratio $\frac{d[1+(y-x) \log (y-x)]}{d y} \operatorname{becomes}$ infinite for $y=x$. It seems that Cauchy's aim here is to contradict Lagrange's results on singular primitive equations. Bottazzini (1990, pp. XLI-XLII) has, however, rightly observed that "for supporting his point Cauchy was forced to 'stretch' the commonly accepted meaning of the concepts he used", since the equation $y=x$ is included in $\log (y-x)=a e^{x}$ only if the substitution $a=-\infty$ is licensed. But this is not all. On the one hand, Lagrange's theory of primitive singular equations is, as such, independent of the consideration of series, and only depends on the algorithm of derivative functions, to the effect that his results concerning singular primitive equations could be established without any appeal to series. On the other hand, according to Lagrange's condition, the equation $y=x$ is not a singular primitive of the equation $y^{\prime}-1-(y-x) \log (y-x)=0$. This is easy to prove. According to this condition, the singular primitive of this equation should result from

$$
\left\{\begin{array}{l}
\log (y-x)=a e^{x} \\
-e^{x}=0
\end{array}\right.
$$

provided its solution $a=\psi(x, y)$ is not a constant. This last condition is not satisfied, however, since, in this case, $a=\psi(x, y)=\frac{\log (y-x)}{0}$. Hence the given derivative equation has, according to Lagrange's condition, no singular primitive.
} 
Let us now view $V$ and $U$ as functions of the variables $y^{\prime}, \ldots, y^{(m)}$ and $y^{\prime}, \ldots, y^{(m-1)}$, respectively, and assume that $V_{y \mid y+z}$ and $U_{y \mid y+z}$ are expanded in power series of $z^{\prime}, \ldots, z^{(m)}$ according to the procedure described in section 4.1 . Let $V^{j}$ and $\stackrel{j}{U}(j=0,1, \ldots)$ be, respectively, the sums of all the terms of order $j$ of the two powers series so obtained, so that: $V_{y \mid y+z}=\sum_{j=0}^{\infty} \stackrel{j}{V}$ and $U_{y \mid y+z}=\sum_{j=0}^{\infty} \stackrel{j}{U}$ (where of course $\stackrel{0}{V}=V$ and $\stackrel{0}{U}=U$ ). Since $\sum_{j=0}^{\infty} \stackrel{j}{V}=\left[\sum_{j=0}^{\infty} \stackrel{j}{U}\right]^{\prime}=\stackrel{\infty}{=} \sum_{j=0}^{j} U^{\prime}$, it will be easy to prove that $\left\{\stackrel{j}{V}=\stackrel{j}{U^{\prime}}\right\}_{j=0}^{\infty}$.

These last equalities provide the necessary and sufficient condition for $V$ to be an exact derivative. It remains to better detail this condition.

If $j=0$, the equality $\stackrel{j}{V}=\stackrel{j}{U^{\prime}}$ holds by hypothesis. Consider the case $j=1$. As $\stackrel{1}{V}=\sum_{k=0}^{m} \Phi_{k} z^{(k)}$ (where $\Phi_{k}$ are appropriate functions of $\left.x, y, y^{\prime}, \ldots, y^{(m)}\right), \stackrel{1}{V}$ is an exact derivative if and only if equality (51) holds. Suppose that this is so. It is easy to prove that the difference $V_{y \mid y+z}-V$ is then an exact derivative.

This is done as follows. From the equalities (50) and (51), it follows that

$$
\stackrel{1}{U}=\sum_{k=0}^{m-1} U^{\prime y^{(k)}} z^{(k)}=\sum_{k=0}^{m} \sum_{h=0}^{k-1}(-1)^{h} \Phi_{k}^{(h)} z^{(k-h-1)}
$$

(where $U^{\prime y^{(k)}}$ is the first derivative of $U$ with respect to $\left.y^{(k)}\right)$ and thus, according to the method of indeterminate coefficients:

$$
U^{\prime} y^{(k)}=\sum_{h=0}^{m-k-1}(-1)^{h} \Phi_{h+k+1}^{(h)},
$$

for any $k(k=0,1, \ldots m-1)$. As the partial derivatives of higher order of $U$ result from the first ones, according to the algorithm of derivatives, these equalities allow us to determine the expansion of the difference $U_{y \mid y+z}-U$, which is the primitive of $V_{y \mid y+z}-V$.

Now, if $V_{y \mid y+z}-V$ is an exact derivative, this is also the case of $V$, since the substitution $z \rightarrow-y$ reduces $V_{y \mid y+z}$ to a function of the only variable $x$, which is certainly an exact derivative. The equality (51) supplies thus a necessary and sufficient condition for $V$ to be an exact derivative, and—as $V^{1}=\sum_{k=0}^{m} \Phi_{k} z^{(k)}$ entails $\Phi_{k}=V^{\prime} y^{(k)}$ for any $k(k=0,1, \ldots, m)$ it can be written under the form:

$$
\sum_{k=0}^{m}(-)^{k}\left[V^{\prime} y^{(k)}\right]^{(k)}=0,
$$

where the derivatives of $V^{\prime} y^{(k)}$ are of course total.

Lagrange has thus proved that if $V=V\left(x, y, y^{\prime}, \ldots y^{(m)}\right)$, Euler and Condorcet's necessary condition of exact integrability (Euler 1744, pp. 71-74; Condorcet 1765, sect. I, pp. 4-35) is also sufficient.

It is easy to understand that if $V=V\left(x, y, y^{\prime}, \ldots y^{(m)}, t, t^{\prime}, \ldots t^{(m)}\right)$ his argument applies separately to $y$ and its derivatives and to $t$ and its derivatives, so that this function is an exact derivative if and only if the equality (54) holds together with an analogous equality where $y$ is replaced by $t$. Lagrange's result is thus general.

4.4. Partial Derivative Equations. The fourth and last example concerns partial derivative equations (Lagrange 1797, arts. 92-96 and 100-107; 1801, continuation of lecture XX, pp. 284-318; 1806, lecture. XX; 1813, arts. I.81-I.84 and I.88-I.95; with the exception of some details, we shall confine ourselves to the account that Lagrange presents in arts. 92-96 and I.81-I.84 of the two editions of the Théorie, respectively). ${ }^{86}$

If $z$ is taken to be a variable independent of $x$ and $y$ (or even a constant), the coefficients of $\xi$ and $o$ in the power-series expansion of $F(x+\xi, y+o, z)$ - that is, the first partial derivatives of $F(x, y, z)$ with respect to $x$ and $y$, under this condition on $z$-can be easily determined according to the equality (39). They differ, of course, from the partial derivatives of $F(x, y, z)$ under the condition that $z$ is a function of $x$ and $y$ implicitly expressed by the equation $F(x, y, z)=0$. Let us respectively denote the former with ' $F^{\prime} x(x, y, z)$ ' and ' $F^{\prime} y(x, y, z)$ ' and the latter with ' $F^{\prime} x\left(x, y, z_{x, y}\right)$ ' and ' $F^{\prime} y\left(x, y, z_{x, y}\right)$ '. ${ }^{87}$ Then:

$$
\begin{aligned}
& F^{\prime x}\left(x, y, z_{x, y}\right)=F^{\prime x}(x, y, z)+F^{\prime z}(x, y, z) z^{\prime x} \\
& F^{\prime y}\left(x, y, z_{x, y}\right)=F^{\prime y}(x, y, z)+F^{\prime z}(x, y, z) z^{\prime y},
\end{aligned}
$$

\footnotetext{
${ }^{86}$ On Lagrange's terminology, see footnote 82 , above.

${ }^{87}$ Lagrange has in fact no elementary symbol for denoting $F^{\prime} x\left(x, y, z_{x, y}\right)$ and $F^{\prime} y\left(x, y, z_{x, y}\right)$.
} 
where $F^{\prime} z(x, y, z)$ is the partial derivative of $F(x, y, z)$ with respect to $z$ under the condition that $z$ is independent of $x$ and $y$, whereas $z^{\prime x}$ and $z^{\prime y}$ are the partial derivatives of $z$ taken as a function of $x$ and $y$. But, if $z$ is taken to be a function of $x$ and $y$ implicitly expressed by $F(x, y, z)=0$, then $F(x+\xi, y+o, z)=0$ holds too, and the method of indeterminate coefficients leads to $F^{\prime x}\left(x, y, z_{x, y}\right)=F^{\prime} y\left(x, y, z_{x, y}\right)=0$. Hence from the equations (55) it follows that:

$$
z^{\prime x}=-\frac{F^{\prime} x(x, y, z)}{F^{\prime} z(x, y, z)} \quad \text { and } \quad z^{\prime y}=-\frac{F^{\prime} y(x, y, z)}{F^{\prime z}(x, y, z)},
$$

which correspond to the equality (21), for the case under consideration.

This is the basic result of Lagrange's reformulation of the part of the calculus dealing with partial differential equations. By generalizing his approach to ordinary derivative equations, he conceives a partial derivative equation as the equation that results if a non-derivative equation $F(x, y, \ldots, z)=0$, involving three or more variables $x, y, \ldots, z$, is combined with the equations obtained by equating to zero the partial derivatives of $F(x, y, \ldots, z)$ with respect to some of these variables. The equalities (56) apply to the simplest case where only three variables are involved. The other cases are analogous.

Consider this simplest one. If the primitive equation $F(x, y, z)=0$ contains two arbitrary constants, they also occur, in general, in the equations $F^{\prime} x\left(x, y, z_{x, y}\right)=0$ and $F^{\prime} y\left(x, y, z_{x, y}\right)=0$ and can thus be eliminated by composing these three equations. Hence, the general solution $F(x, y, z)=0$ of a first order partial differential equation involving the variables $x, y, z, z^{\prime}-i . e$. , in Lagrange parlance, the "complete primitive equation" 88 of this equation- contains two arbitrary constants.

But singular solutions exist here too, as Lagrange shows by considering a function $F(x, y, z, a, b)$ of five variables $x, y, z, a, b$, and assuming that $z, a$, and $b$ are functions of $x$ and $y$. In this case (Lagrange 1801, p. 296; 1806, p. 367-369), the equations $F^{\prime x}\left(x, y, z_{x, y}, a_{x, y}, b_{x, y}\right)=0$ and $F^{\prime y}\left(x, y, z_{x, y}, a_{x, y}, b_{x, y}\right)=0$ have respectively the form

$$
\begin{aligned}
& F^{\prime x}(-)+F^{\prime z}(-) z^{\prime x}+F^{\prime a}(-) a^{\prime x}+F^{\prime} b(-) b^{\prime x}=0 \\
& F^{\prime y}(-)+F^{\prime z}(-) z^{\prime y}+F^{\prime a}(-) a^{\prime y}+F^{\prime} b(-) b^{\prime y}=0,
\end{aligned}
$$

where the dashes stand for the sequence ' $x, y, z, a, b$ '. If $F^{\prime} a(-)=F^{\prime} b(-)=0$, the composition of these equations with their primitive gives the same partial derivative equation obtained by composing the equation $F(x, y, z)=0$ with the corresponding equations $F^{\prime x}\left(x, y, z_{x, y}\right)=0$ and $F^{\prime y}\left(x, y, z_{x, y}\right)=0$. Hence, to obtain the singular solution of a partial derivative equation involving the variables $x, y, z, z^{\prime}$, it suffices to take the arbitrary constants $a$ and $b$ occurring in the complete primitive equation as two functions of $x$ and $y$, and to require that $F^{\prime a}(-)=F^{\prime} b(-)=0$. Lagrange calls such a solution the 'singular primitive equation'.

Now, let $b$ be a function of $a$, this last variable being a function of $x$ and $y$, again. Suppose in particular that $b=\varphi(a)$. Equations (57) become

$$
\begin{aligned}
& F^{\prime x}(-)+F^{\prime} z(-) z^{\prime x}+\left[F^{\prime a}(-)+F^{\prime} \varphi(a)(-) \varphi^{\prime}(a)\right] a^{\prime x}=0 \\
& F^{\prime y}(-)+F^{\prime} z(-) z^{\prime y}+\left[F^{\prime a}(-)+F^{\prime} \varphi(a)(-) \varphi^{\prime}(a)\right] a^{\prime y}=0 .
\end{aligned}
$$

Thus, if $a$ satisfies the condition

$$
F^{\prime a}(-)+F^{\prime} \varphi(a)(-) \varphi^{\prime}(a)=0
$$

the composition of the these equations with $F(x, y, z, a, b)=0$ produces the same partial derivative equation obtained by composing $F(x, y, z)=0$ with $F^{\prime x}\left(x, y, z_{x, y}\right)=0$ and $F^{\prime} y\left(x, y, z_{x, y}\right)=0$. As this does not depend on the particular nature of the function $\varphi(a)$, and $a$ is supposed to be a function of $x$ and $y$, it follows that such a partial derivative equation has a primitive which contains an arbitrary function of $x$ and $y$ and no arbitrary constant. Lagrange calls it the 'general primitive equation'.

As a simple example consider the equation $F(x, y, z)=z-a x-b y-c=0$ where $a, b$ and $c$ are constants and $z$ is taken to be a function of $x$ and $y$ implicitly expressed by this equation. The equations $F^{\prime} x\left(x, y, z_{x, y}\right)=0$ and $F^{\prime} y\left(x, y, z_{x, y}\right)=0$ are $z^{\prime x}=a$ and $z^{\prime}=b$, and, by composition, one obtains the partial derivative equation $z-z^{\prime x} x-z^{\prime} y-c=0$, of which $z-a x-b y-c=0$ is the complete primitive. Suppose that also $a$ and $b$ are functions of $x$ and $y$. Since, in this case, $F^{\prime} a(x, y, z, a, b)=-x$, and $F^{\prime} b(x, y, z, a, b)=-y$, to obtain the singular primitive, it is enough to impose the condition $x=y=0$ on this complete primitive, which gives $z-c=0$. Suppose now that $b=\varphi(a)$, and $a$ is a function of $x$ and $y$. Since, in this case, $F^{\prime} a(x, y, z, a, b)+F^{\prime} \varphi(a)(-) \varphi^{\prime}(a)=x+y \varphi^{\prime}(a)$, to obtain the general primitive, one also has to impose the condition $x+y \varphi^{\prime}(a)=0$ on this same complete primitive. But, from this condition it follows that $a=\varphi^{\prime-1}\left(-\frac{x}{y}\right)$ and $b=\varphi\left(\varphi^{\prime-1}\left(-\frac{x}{y}\right)\right)$. Hence, the general primitive is $z-y \psi\left(\frac{x}{y}\right)-c=0$, where $\psi\left(\frac{x}{y}\right)=\frac{x}{y} \varphi^{\prime-1}\left(-\frac{x}{y}\right)+\varphi\left(\varphi^{\prime-1}\left(-\frac{x}{y}\right)\right)$ is an arbitrary function of $\frac{x}{y}$.

\footnotetext{
${ }^{88}$ For the introduction of this and the following terms, see Lagrange (1801), p. 296-298; (1806), p. 369-371.
} 
4.5. Returning to Lagrange's Notion of Function. The previous examples make it clear that, in reformulating the calculus, Lagrange is forced to deal with functions in a way that is at odds with the compositional conception, and, more generally, with the identification of functions with appropriate expressions.

Not only, he does identify functions with generic variables depending on other variables and merely expressed by generic symbols submitted to an appropriate algebra, he is also forced to acknowledge the existence of functions that can only be characterized as the functions expressed by such symbols. This is manifestly the case for the arbitrary functions $\varphi(a)$ and $\psi\left(\frac{x}{y}\right)$ involved in his account of the general primitives of a partial derivative equation (see section 4.4, above). What makes these functions arbitrary is not merely that they enter into a general argument that does not depend, as such, on their being some particular expressions (or some quantities expressed by some particular expressions). These functions are arbitrary also because this argument does not require that they be expressions (or quantities expressed by some expressions). For this argument to work, it is enough to take these functions to be atomic symbols for quantities that satisfy an appropriate algebra (or quantities denoted by such symbols).

This is a new and fairly clear example of the tensions between Lagrange's ideal of purity and the actual deployment of his theory. Though he cautiously avoids, in his treatises, the issues raised by Euler's famous 1765 memoir on "discontinuous functions" (Euler 1765), and the debate generated by it, his efforts to recover the totality of calculus within this theory are such that the necessity of considering functions merely as quantities "somehow determined through some variable" (Euler 1765, p. 3) arises naturally. ${ }^{89}$

More generally, this same difficulty affects Lagrange's reformulation of the integral calculus as such, since his notions of function and derivative seem too strict to allow each function to have a primitive. For him, the passage from a given function to his primitive relies on an "operation" which can be regarded as "the inverse" of the derivation, and that "can always be performed through series", by appealing to the method of indeterminate coefficients. ${ }^{90}$ But if the operation of derivation is viewed as the operation that leads from a given function $f(x)$ to the coefficient of $\xi$ in the power-series expansion of $f(x+\xi)$, its inverse can only be seen as the operation leading from a given function $f(x)$ to a function $g(x)$ such that $f(x)$ is the coefficient of $\xi$ in the power-series expansion of $g(x+\xi)$ (see footnote (83) above). But how then can one determine the primitive of a function $f(x)$ that can only be integrated by series?

Lagrange could have answered this question in different ways, but no one of them would have been in good order with respect to the principles of his theory. He could have admitted that a series is in itself a function, and taken a series to be the primitive of $f(x)$. But this is something that he does not seem to have been ready to admit, and that is in any case openly at odd with his notion of function. He could have argued that the primitive of $f(x)$ is unknown though having a known expansion. But, he should then have explained what sort of function this unknown function might have been, which would have been hard to do on the base of his notion of function. Finally, he could have granted that the primitive of $f(x)$ is not a function in turn, or that $f(x)$ has no primitive at all. But in this way, his theory would have departed from a generally admitted assumption of the differential calculus. ${ }^{91}$

\section{THE REMAINDER THEOREM}

No appropriate appreciation of Lagrange's theory of analytical functions can omit consideration of his remainder theorem. This is not only generally seen to be the major mathematical achievement of the Théorie and the Leçons; it is also the basic result that the geometric and mechanical applications of such a theory depend on.

Modern calculus treatises often state the theorem as follows:

Theorem 5 (Modern Remainder theorem). If $f(z)$ is a function of a real variable $z$ differentiable up to order $h+1$ in a right neighborhood of $x$ including $x$ itself (but possibly not differentiable at order $h+1$ at $x$ ) and $z$ belongs to this

\footnotetext{
${ }^{89}$ On the well-known debate generated by Euler's memoir, see Truesdell (1995), pp. 237-300; Grattan-Guinness (1970), 1-21; Dhombres (1988); Bottazzini (1986), pp. 21-33; Panza (1992), 256-264. The issues of this debate were hard to reconcile with the structure of algebraic analysis (GrattanGuinness 1970, pp. 6-11). This explains why, both in his 1765 memoir and in other memoirs on the same matter-like Euler (1749) and (1753)-, Euler often and crucially relies on geometric interpretations of his formalism.

${ }^{90}$ See Lagrange 1797, art. 58; 1813, art. I.45:

In the foregoing examples, we looked for the derivative equation and then determined the value of the primitive function $y$ through this equation. Since this last operation is [...] the inverse of that through which one comes down from the primitive function to the derivative ones, it can always been performed through series, by employing, as we did, a series with indeterminate coefficients, and by drawing a separate equation from any term affected by a power of $x$. In this way, one determines the coefficient one after the others, and one has often the advantage of grasping the general law that governs these coefficients.

Dans les exemples précédents, nous avons cherché l'équation dérivée et nous avons ensuite déterminé par cette équation la valeur de la fonction primitive $y$. Cette dernière opération est [...] l'inverse de celle par laquelle on descend de la fonction primitive aux fonctions dérivées; elle peut toujours s'exécuter par le moyen des séries, en employant, comme nous l'avons fait, une série avec des coefficients indéterminés, et faisant des équations séparées des termes affectés de chaque puissance de $x$. De cette manière, on détermine les coefficients les uns par les autres, et l'on a souvent l'avantage d'apercevoir la loi générale qui règne entre ces coefficients.

${ }^{91}$ For other considerations on this matter, see Fraser (1987), p. 40.
} 
neighborhood and differs from $x$, then the difference

$$
f(z)-\sum_{k=0}^{h} \frac{d^{k}}{d z^{k}}[f(z)]_{z=x} \frac{(z-x)^{k}}{k !}
$$

between this function and the associated Taylor polynomial of order $h$ is equal to

$$
\frac{d^{h+1}}{d z^{h+1}}[f(z)]_{z=\lambda} \frac{(z-x)^{h+1}}{(h+1) !},
$$

for some $\lambda$ strictly between $x$ and $z$.

Mutatis mutandis, this statement already occurs, for example, in Laplace's Théorie analytique des probabilités (Laplace 1812, pp. 175-176) and in Lacroix's Traité du calcul différentiel et du calcul intégral (Lacroix 1810-1819, vol. III, p. 399), where it is proved through appropriate integrations by parts. ${ }^{92}$ Its importance rests on the possibility it provides of proving that the Taylor series of a suitable class of functions converges to these functions in an appropriate interval.

Lagrange's result is essentially different: $i$ ) it involves derivative functions in Lagrange's sense, rather than differential ratios (as in the versions of Laplace and Lacroix) or derivative functions in modern sense (as in the modern version) $;^{93}$ ii) it concerns the remainder of the power-series expansion $\sum_{k=0}^{h} \frac{f^{(k)}(x)}{k !} \xi^{k}=\sum_{k=0}^{h} \frac{f^{(k)}(x)}{k !}(z-x)^{k}$ of the function $f(x+\xi)=$ $f(x+(z-x))=f(z)$, rather than the difference (60); iii) it presupposes the convergence of the relevant series, to the effect that it cannot be used to prove that this series converges in appropriate cases.

In our understanding, Lagrange's result is then the following:

Theorem 6 (Lagrange's Remainder theorem). If $\xi$ is a positive increment such that the series $\sum_{k=0}^{\infty} \frac{f^{(k)}(x)}{k !} \xi^{k}$ converges to $f(x+\xi)$, then, for any order $h(h=0,1, \ldots)$ :

$$
f(x+\xi)=\sum_{k=0}^{h} \frac{f^{(k)}(x)}{k !} \xi^{k}+\frac{\xi^{h+1}}{(h+1) !} f^{(h+1)}(x+j),
$$

where $j$ is an appropriate increment such that $0 \leq j \leq \xi$.

Lagrange is quite explicit in asserting that equality (62) holds (Lagrange 1797, art. 53; 1813, art. I.40), but he is vague about the conditions under which it does. We argue the conditions are those expressed in theorem 6 , because of the arguments Lagrange uses to prove the theorem: namely, it seems these arguments hold only if $\sum_{k=0}^{\infty} \frac{f^{(k)}(x)}{k !} \xi^{k}$ converges to $f(x+\xi)$ around $\xi=0$, so that Lagrange's remainder theorem cannot be part of a more general argument intended to prove that the power-series expansion of a function $f(x+\xi)$ converges to this same function around $\xi=0$.

We have said that the remainder theorem is the basic result on which the geometric and mechanical applications of Lagrange's theory depend, and that its proof depends on the convergence of the power-series expansion of $f(x+\xi)$ to this same function around $\xi=0$. This is clearly inconsistent with claiming that the convergence of the power-series expansion of $f(x+\xi)$ to this same function around $\xi=0$ is a sufficient condition for applying the theory of analytical functions to the solution of geometric and mechanical problems. We reject this last claim, indeed.

According to us, the examination of the arguments Lagrange relies on in the Théorie to justify these applications shows that these arguments not only require the convergence of the power-series expansion of $f(x+\xi)$ to this same function around $\xi=0$ and/or the evaluation of the series $\sum_{k=h+1}^{\infty} \frac{f^{(k)}(x)}{k !} \xi^{k}$, but also rely on the admission that that this series can be expressed under the form of a product like $\frac{\xi^{h+1}}{(h+1) !} f^{(h+1)}(x+j)$, as the equality (62) prescribes. ${ }^{94}$

\footnotetext{
${ }^{92}$ For a modern version of Laplace's and Lacroix's proof, see Giusti (1983), vol. I, pp. 235-237. Giusti obtains the equality
}

$$
f(z)-\sum_{k=0}^{h} \frac{d^{k}}{d z^{k}}[f(z)]_{z=x} \frac{(z-x)^{k}}{k !}=\frac{1}{h !} \int_{x}^{z}(z-t)^{h} \frac{d^{h+1}}{d z^{h+1}}[f(z)]_{z=t} d t
$$

though induction on $h$ and integration by parts, then observes that, since $\frac{d^{h+1}}{d z^{h+1}}[f(z)]$ is supposed to be differentiable in the relevant neighborhood of $x$, it is also continuous, and so:

$$
\frac{1}{h !} \int_{x}^{z}(z-t)^{h} \frac{d^{h+1}}{d z^{h+1}}[f(z)]_{z=t} d t=\frac{1}{h !} \frac{d^{h+1}}{d z^{h+1}}[f(z)]_{z=\lambda} \int_{x}^{z}(z-t)^{h} d t=\frac{d^{h+1}}{d z^{h+1}}[f(z)]_{z=\lambda} \frac{(z-x)^{h+1}}{(h+1) !} .
$$

for some $\lambda$ strictly between $x$ and $z$.

${ }^{93}$ Our use of the differential notation in formulas (60) and (61), and in footnote 92 aims to emphasize this difference.

${ }^{94}$ In our view, the series $\sum_{k=h+1}^{\infty} \frac{f^{(k)}(x)}{k !} \xi^{k}$ is precisely what should count, in Lagrange's theory, as the remainder of the power-series expansion of $f(x+\xi)$. The distinction between the evaluation of such a remainder and the way this remainder is expressed is explicitly introduced by Lagrange himself in the Leçons: 


\subsection{Tangents, Areas, Speed and Acceleration. Examples will be useful for clarification.}

5.1.1. Tangents. Let us begin with Lagrange's treatment of the problem of tangents (Lagrange 1797, arts. 109-113; Lagrange 1813, arts. II.2-II.6; Lagrange's argument has been reconstructed in Grabiner 1990, pp. 159-160bis).

Let $u=\varphi(v), u=\phi(v)$ and $u=\psi(v)$ be three functions expressing three different curves that meet each other in a point $v=x$, so that: $\varphi(x)=\phi(x)=\psi(x)$. For simplicity, suppose that in a right neighborhood $I_{x}^{>}$of this point, such that the power-series expansions of $\varphi(x+\xi), \phi(x+\xi)$ and $\psi(x+\xi)$ converge to these functions if $x+\xi \in I_{x}^{>}$, these curves are all increasing, their ordinates are positive, and the ordinate of the first is smaller than or equal to the ordinates of the two others (appropriate variants of the following argument apply in other cases).

Then, let $\Delta_{1}$ and $\Delta_{2}$ be the (non-negative) differences between $\phi(x+\xi)$ and $\varphi(x+\xi)$ and between $\psi(x+\xi)$ and $\varphi(x+\xi)$, respectively. As $\varphi(x)=\phi(x)=\psi(x)$, from theorems 4 and 6 it follows that:

$$
\begin{aligned}
& \Delta_{1}=\phi(x+\xi)-\varphi(x+\xi)=\xi\left[\phi^{\prime}(x)-\varphi^{\prime}(x)\right]+\frac{\xi^{2}}{2}\left[\phi^{\prime \prime}\left(x+j_{[\phi], 2}\right)-\varphi^{\prime \prime}\left(x+j_{[\varphi], 2}\right)\right] \\
& \Delta_{2}=\psi(x+\xi)-\varphi(x+\xi)=\xi\left[\psi^{\prime}(x)-\varphi^{\prime}(x)\right]+\frac{\xi^{2}}{2}\left[\psi^{\prime \prime}\left(x+j_{[\psi], 2}\right)-\varphi^{\prime \prime}\left(x+j_{[\varphi], 2}\right)\right],
\end{aligned}
$$

where $j_{[\varphi], 2}, j_{[\phi], 2}$ and $j_{[\psi], 2}$ are three increments depending on $x$ and on the nature of functions $\varphi(v), \phi(v), \psi(v)$, but in any case belonging to $[0, \xi]$.

Let us assume that $\varphi^{\prime}(x)=\phi^{\prime}(x)$. Since $\Delta_{2}$ is non-negative, $\psi^{\prime}(x)-\varphi^{\prime}(x)$ is too. If it were positive, there would be a positive quantity $\delta$ small enough for

$$
\Delta_{1}-\Delta_{2}=\frac{\xi^{2}}{2}\left[\phi^{\prime \prime}\left(x+j_{[\phi], 2}\right)-\psi^{\prime \prime}\left(x+j_{[\psi], 2}\right)\right]-\xi\left[\psi^{\prime}(x)-\varphi^{\prime}(x)\right]<0,
$$

whenever $0<\xi \leq \delta$. But in $I_{x}^{>}$the curve expressed by the function $u=\psi(v)$ lies between the curves expressed by the functions $u=\varphi(v)$ and $u=\phi(v)$ if and only if $\Delta_{1} \geq \Delta_{2}$. So, in $I_{x}^{>}$, the former curve lies between the latter ones only if $\psi^{\prime}(x)=\varphi^{\prime}(x)$.

It is thus enough to suppose that the curves expressed by the functions $u=\phi(v)$ and $u=\psi(v)$ are two straight lines of equations $u=p_{[\phi]} v+q_{[\phi]}$ and $u=p_{[\psi]} v+q_{[\psi]}$, respectively, to conclude that, in $I_{x}^{>}$, the latter of these straight lines lies between the curve expressed by the function $u=\varphi(v)$ and the former straight lines only if $p_{[\phi]}=p_{[\psi]}$. But, as long as these straight lines meet each other in the point $v=x$, this means that they coincide. Therefore, there is no straight line passing through the point $v=x$ that, in $I_{x}^{>}$, lies between the curve expressed by the function $u=\varphi(v)$ and the straight line passing through this point whose slope is equal to $\varphi^{\prime}(x)$. This last line is therefore the tangent to the curve at $v=x$.

5.1.2. Areas. Consider now, Lagrange's treatment of the problem of areas (Lagrange 1797, art. 134; 1813, art. II.27; Lagrange's argument has been reconstructed in Grabiner 1981a, p. 157; Grabiner 1990, pp. 160bis-162).

Let $u=\varphi(v)$ and $u=\phi(v)$ be two functions expressing respectively a curve referred to a system of orthogonal coordinates and the area under this curve (taken starting from a certain fixed point). For simplicity, suppose that in a right neighborhood $I_{x}^{>}$of a generic point $v=x$ such that the power-series expansions of $\varphi(x+\xi)$ and $\phi(x+\xi)$ converge to these functions if $x+\xi \in I_{x}^{>}$, the ordinates of $\varphi(v)$ are non-negative and increasing (appropriate variants of the following argument apply in other cases). ${ }^{95}$

The difference $\phi(x+\xi)-\phi(x)$ is thus non-negative as well, and

$$
\xi \varphi(x) \leq \phi(x+\xi)-\phi(x) \leq \xi \varphi(x+\xi),
$$

whenever $x+\xi \in I_{x}^{>}$. According to theorem 6, this condition reduces to

$$
0 \leq \phi^{\prime}(x)-\varphi(x) \leq \xi\left[\varphi^{\prime}\left(x+j_{[\varphi], 1}\right)-\frac{\phi^{\prime \prime}\left(x+j_{[\phi], 2}\right)}{2}\right],
$$

In the solution I advanced for this problem in [...][the Théorie], I begun by searching the exact expression of the remainder of the series, then I determined the limits of this expression. But one can immediately find these limits in a more elementary ald also more rigorous way.

Dans la solution que j'ai donnée de ce problème dans [...] [la Théorie], j'ai commencé par chercher l'expression exacte du reste de la série, ensuite j'ai déterminé les limites de cette expression. Mais on peut trouver immédiatement ces limites d'une manière plus élémentaire, et également rigoureuse.

(Lagrange 1801, p. 66; 1806, p. 86.)

${ }^{95}$ Lagrange explicitly supposes that any function is made up of monotonic pieces. After having presented his argument, he writes:

We supposed [...] that the ordinates increased or diminished from $f(x)$ up to $f(x+\xi)$ : this condition would not obtain if there were a maximum or minimum between these two ordinates. But, since one can take the interval $\xi$ as small as one likes, it is clear that one will be always able to make the second ordinate falls before the maximum or minimum, to the effect that the conclusion we have drawn will remain the same.

Nous avons supposé $[\ldots]$ que les ordonnées allaient en augmentant ou en diminuant depuis $f(x)$ jusqu'à $f(x+\xi)$ : cette condition n'aurait pas lieu s'il y avait entre ces deux ordonnées un maximum ou un minimum; mais, comme on peut prendre l'intervalle $\xi$ assez petit que l'on veut, il est clair qu'on pourra toujours faire tomber la seconde ordonnée $f(x+\xi)$ en deçà du maximum ou du minimum, et que, par consequant, la conclusion que nous avons tirée demeurera toujours la même.

On this matter, see Dugac (2003), p. 76. 
where $j_{[\varphi], 1}$ and $j_{[\phi], 2}$ are two increments depending on $x$ and on the nature of the functions $\varphi(v)$ and $\phi(v)$, but in any case belonging to $[0, \xi]$. But this condition holds for any non-negative $\xi$ only if $\phi^{\prime}(x)=\varphi(x)$. Indeed if it were not it would be enough that

$$
\xi<\frac{\phi^{\prime}(x)-\varphi(x)}{\varphi^{\prime}\left(x+j_{[\varphi], 1}\right)-\frac{1}{2} \phi^{\prime \prime}\left(x+j_{[\phi], 2}\right)},
$$

for condition (66) to not hold. Hence, $\phi^{\prime}(x)=\varphi(x)$.

5.1.3. Speeds and Accelerations of Rectilinear Motions. Finally, consider Lagrange's treatment of speeds and accelerations of a rectilinear motion (Lagrange 1797, art. 188; Lagrange (1813), art. III.4; see also Panza 1991-1992, pp. 184-187 of vol. 45).

Let $u=\varphi(v)$ be a function expressing the space $u$ covered in time $v$ by a point moving uniformly in a straight line and $v=t$ the initial instant of motion. It follows that $\varphi(t)=0$ and that, starting from this moment, $\varphi(v)$ is non-negative and increasing. Hence, $\varphi(t+\theta)-\varphi(t)=\varphi(t+\theta)$ is non-negative too, and expresses the space covered in a (positive) time $\theta$ starting from this instant. Lagrange claims that $\theta$ can be taken small enough for the movement expressed by the two first terms of the power-series expansion of $\varphi(t+\theta)$ to "draw nearer the real motion than any other [rectilinear] movement composed by a uniform movement and a uniformly accelerated movement", so that "the term $\theta \varphi^{\prime}(t)$ expresses all that which can there be of uniform [tout ce qu'il peut y avoir d'uniforme] in the proposed motion considered at the beginning of time $\theta$ ", whereas "the term $\frac{\theta^{2}}{2} \varphi^{\prime \prime}(t)$ expresses [...] all that which could there be of uniformly accelerated [tout ce qu'il peut y avoir d'uniformement accéléré] in this motion."

The mechanical concerns of his argument do not interest us here. Consider only the part of it which is supposed to prove that, for any $a$ and $b$ independent of $\theta$, respectively different from $\varphi^{\prime}(t)$ and $\frac{\varphi^{\prime \prime}(t)}{2}$, and such that $a \theta+b \theta^{2}$ is non-negative, there is a quantity $\vartheta$ small enough for

$$
\left|[\varphi(t+\theta)-\varphi(t)]-\left[a \theta+b \theta^{2}\right]\right|>\left|[\varphi(t+\theta)-\varphi(t)]-\left[\theta \varphi^{\prime}(t)+\frac{\theta^{2}}{2} \varphi^{\prime \prime}(t)\right]\right|,
$$

whenever $0<\theta \leq \vartheta$.

According to theorem 6 , this reduces to the claim that, under the above conditions,

$$
\left|\left[\varphi^{\prime}(t)-a\right]+\left[\frac{\varphi^{\prime \prime}(t)}{2}-b\right] \theta+\frac{\varphi^{\prime \prime \prime}\left(t+j_{[\varphi], 2}\right)}{3 !} \theta^{2}\right|>\left|\frac{\varphi^{\prime \prime \prime}\left(t+j_{[\varphi], 3}\right)}{3 !} \theta^{2}\right|,
$$

where $j_{[\varphi], 3}$ is an increment depending on $t$ and on the nature of function $\varphi(v)$, but in any case belonging to $[0, \theta]$. According to Lagrange, this "can be easily proved by an argument similar" to that used to prove the extension up to the third order of the result on which his solution of the problem of tangents is based (Lagrange 1797, art. 111; 1813, art. II.4).

Suppose that $u=\phi(v)$ and $u=\psi(v)$ are two new functions such that $\varphi(t)=\phi(t)=\psi(t), \varphi^{\prime}(t)=\psi^{\prime}(t)$, and $\varphi^{\prime \prime}(t)=$ $\psi^{\prime \prime}(t)$. Suppose also that $\Delta_{1}=\varphi(t+\theta)-\phi(t+\theta)$, and $\Delta_{2}=\varphi(t+\theta)-\psi(t+\theta)$. From theorem 6 one derives:

$$
\begin{aligned}
& \Delta_{1}=\theta\left[\varphi^{\prime}(t)-\phi^{\prime}(t)\right]+\frac{\theta^{2}}{2}\left[\varphi^{\prime \prime}(t)-\phi^{\prime \prime}(t)\right]+\frac{\theta^{3}}{3 !}\left[\varphi^{\prime \prime \prime}\left(t+j_{[\varphi], 3}\right)-\phi^{\prime \prime \prime}\left(t+j_{[\phi], 3}\right)\right] \\
& \Delta_{2}=\frac{\theta^{3}}{3 !}\left[\varphi^{\prime \prime \prime}\left(t+j_{[\varphi], 3}\right)-\psi^{\prime \prime \prime}\left(t+j_{[\psi], 3}\right)\right]
\end{aligned}
$$

where $j_{[\phi], 3}$ and $j_{[\psi], 3}$ are two increments depending on $t$ and on the nature of the functions $\phi(v)$ and $\psi(v)$, but in any case belonging to $[0, \theta]$. The extension Lagrange is clearly referring consists in the claim that there is a quantity $\vartheta$ small enough for $\left|\Delta_{1}\right|>\left|\Delta_{2}\right|$ whenever $0<\theta \leq \vartheta$.

Without considering the absolute values (that is, assuming $\Delta_{1}$ and $\Delta_{2}$ to be both non-negative), Lagrange argues that for it to happen, it is enough that

$$
\left[\varphi^{\prime}(t)-\phi^{\prime}(t)\right]+\frac{\theta}{2}\left[\varphi^{\prime \prime}(t)-\phi^{\prime \prime}(t)\right]>\frac{\theta^{2}}{3 !}\left[\phi^{\prime \prime \prime}\left(t+j_{[\phi], 2}\right)-\psi^{\prime \prime \prime}\left(t+j_{[\psi], 3}\right)\right],
$$

which, he says, "is obviously possible when $\varphi^{\prime}(t)-\phi^{\prime}(t)$ does not vanish", and, if $\varphi^{\prime}(t)-\phi^{\prime}(t)=0$, "is still manifestly possible, by decreasing the value of $\theta$ as much as one wishes whenever $\varphi^{\prime \prime}(t)-\phi^{\prime \prime}(t)$ does not vanish."

If this claim is applied to the problem of speeds and accelerations of rectilinear motions-for which $\phi(t+\theta)=$ $a \theta+b \theta^{2}$ and $\psi(t+\theta)=\theta \varphi^{\prime}(t)+\frac{\theta^{2}}{2} \varphi^{\prime \prime}(t)$ - the inequality (71) reduces to

$$
\left[\varphi^{\prime}(t)-a\right]+\theta\left[\frac{\varphi^{\prime \prime}(t)}{2}-b\right]>0
$$

Now, when referred to this inequality, Lagrange's first claim is true: if $\varphi^{\prime}(t)>a$, it is certainly possible that $\theta$ be taken small enough, though positive, for $\left[\varphi^{\prime}(t)-a\right]>\theta\left[b-\frac{\varphi^{\prime \prime}(t)}{2}\right]$. But this is not so for his second claim, since, in this case, $\varphi^{\prime \prime}(t)-\phi^{\prime \prime}(t)=0$, and nothing ensures, in general, that $\theta\left[\frac{\varphi^{\prime \prime}(t)}{2}-b\right]>0$. Hence, taken as such, Lagrange's argument is flawed, and should be reformulated, by relying on the fact that any polynomial $P(z)$ of a real variable $z$ takes the sign of its 
first term when the absolute value of $z$ becomes small enough (Panza 1991-1992, p. 186 of vol. 45). However, insofar as such a reformulation still involves the equality (71), the role of the remainder theorem in the argument remains the same.

5.1.4. Reflecting on the foregoing examples. The arguments Lagrange uses in the three examples we've just seen do not explicitly appeal to the convergence of the power-series expansion of $f(x+\xi)$ to this same function around $\xi=0$, and such convergence is in fact not enough for they to be correct. ${ }^{96}$ The crucial result on which these arguments are based is, rather, the remainder theorem.

For any non-negative integer $h$, let $R_{[f], h+1}(x, \xi)$ be the remainder of order $h(h=1,2, \ldots)$ of the power-series expansion of a function $f(x+\xi)$, i. e. the series $\sum_{k=h+1}^{\infty} \frac{f^{(k)}(x)}{k !} \xi^{k}$. The role of this theorem in the three foregoing arguments is that of ensuring, respectively, that, for any pair of functions $f(x+\xi)$ and $g(x+\xi)$ :

- i) $R_{[f], 2}(x, \xi)$ and $R_{[g], 2}(x, \xi)$ are such that there is a quantity $\delta$ small enough that, if $0<\xi \leq \delta$ and $g^{\prime}(x)-f^{\prime}(x)$ is positive, then

$$
\left|R_{[f], 2}(x, \xi)-R_{[g], 2}(x, \xi)\right|<\xi\left[g^{\prime}(x)-f^{\prime}(x)\right]
$$

- ii) $R_{[f], 2}(x, \xi)$ and $R_{[g], 1}(x, \xi)$ are such that

$$
0 \leq \xi\left[f^{\prime}(x)-g(x)\right] \leq \xi R_{[g], 1}(x, \xi)-R_{[f], 2}(x, \xi),
$$

for any positive $\xi$ belonging to an appropriate right neighborhood of 0 only if $f^{\prime}(x)-g(x)=0$;

- iii) $R_{[f], 3}(x, \xi)$ is such that there is a quantity $\delta$ small enough that, if $0<\xi \leq \delta$, and $a$ and $b$ are independent of $\theta$ and respectively different from $f^{\prime}(x)$ and $\frac{f^{\prime \prime}(x)}{2}$, then

$$
\left|\xi\left[f^{\prime}(x)-a\right]+\xi^{2}\left[\frac{f^{\prime \prime}(x)}{2}-b\right]+R_{[f], 3}(x, \xi)\right|>\left|R_{[f], 3}(x, \xi)\right| .
$$

It thus seems that, according to Lagrange, the remainder theorem ensures that, when $\xi$ approaches zero (though remaining positive), $R_{[f], h+1}(x, \xi)$ behaves as a product like $\xi^{h+1} \Lambda_{[f], h+1}$, where $\Lambda_{h+1}$ is a finite factor independent of $\xi$. When $\xi$ approaches zero, the inequalities (73), (74) and (75) would then be equivalent to these:

$$
\begin{gathered}
\left|\Lambda_{[f], 2}-\Lambda_{[g], 2}\right|<\frac{A-B}{\xi} \\
0 \leq \frac{A-B}{\xi} \leq \Lambda_{[g], 1}-\Lambda_{[f], 2}, \\
\left|\frac{A}{\xi^{2}}+\frac{B}{\xi}+\Lambda_{[f], 3}\right|>\left|\Lambda_{[f], 3}\right|
\end{gathered}
$$

where $A$ and $B$ are finite quantities that are independent of $\xi$.

We know the remainder theorem does not in fact ensures this, since the increment $j_{[f], h+1}$ is supposed to belong to an interval whose upper bound is $\xi$ and is thus actually not independent of this increment. But for our purpose it is more important to wonder why Lagrange does not merely base his arguments on the equality

$$
R_{[f], h+1}(x, \xi)=\sum_{k=h+1}^{\infty} \frac{f^{(k)}(x)}{k !} \xi^{k}=\xi^{h+1} \sum_{k=0}^{\infty} \frac{f^{(h+k+1)}(x)}{(h+k+1) !} \xi^{k} .
$$

His choice of appealing to the remainder theorem suggests that he was convinced that the identification of the series $\sum_{k=h+1}^{\infty} \frac{f^{(k)}(x)}{k !} \xi^{k}$ with a product like $\xi^{h+1} \Lambda_{[f], h+1}$ is only guaranteed by the equality

$$
\sum_{k=0}^{\infty} \frac{f^{(h+k+1)}(x)}{(h+k+1) !} \xi^{k}=\frac{f^{(h+1)}(x+j)}{(h+1) !} .
$$

${ }^{96}$ Lagrange himself claims that this is so. At the end of the proof of the remainder theorem in the Théorie, he writes:

The perfection of the methods of approximation in which some series are employed not only depends on the convergence of the series, but also on the possibility of estimating the error resulting from the neglected terms. Concerning this matter, one could say that almost all methods that are used in the solution of geometric and mechanical problems are very imperfect. The foregoing theorem will be useful, in many occasions, in order to confer to these methods the perfection of which they lack and whiteout which it is often dangerous to use them.

La perfection des méthodes d'approximation dans lesquelles on emploie les séries dépend non seulement de la convergence des séries, mais encore de ce qu'on puisse estimer l'erreur qui résulte des termes qu'on néglige, et à cet égard on peut dire que presque toutes les méthodes d'approximation dont on fait usage dans la solution des problèmes géométriques et mécaniques sont encore très-imparfaites. Le théorème précédent pourra servir, dans beaucoup d'occasions, à donner à ces méthodes la perfection qui leur manque et sans laquelle il est souvent dangereux de les employer.

(Lagrange 1797, art. 53; 1813, art. I.40) 
But, why does Lagrange believe this? We suggest the following answer: to feel justified in considering the series $\sum_{k=0}^{\infty} \frac{f^{(h+k+1)}(x)}{(h+k+1) !} \xi^{k}$ in the context of an argument concerned with particular quantities, Lagrange requires to have proved that this series reduces to a quantity expressed by a suitable, finitary expression, $i$. $e$. to an algebraic quantity or function (of $\xi$ ). This is precisely the content of the remainder theorem.

5.2. Lagrange's Proofs of the Remainder Theorem. Having clarified the role of the remainder theorem in Lagrange's theory, we turn to its proofs. We speak of proofs in the plural, since Lagrange proves this theorem in two different ways in the Théorie and in the Leçons. Furthermore, in the second edition of the Théorie, he adjusts the argument provided in the first.

5.2.1. The theorem of the sufficiently small increment. The argument advanced in the first edition of the Théorie explicitly relies on a result proved at the beginning of the treatise, right after the proof of the power-series expansion theorem (Lagrange 1797, arts. 11-15; 1813, arts. I.3-I.7); we suggest to term it 'theorem of the sufficiently small increment'. Lagrange states it as follows (Lagrange 1797, art. 14; 1813, art. I.6; the same result is stated twice in these articles; we quote the first occurrence; a third equivalent statement occurs in articles 15 and. I.7 of the same treatises, respectively):

Theorem 7 (Theorem of the sufficiently small increment). $\quad[\ldots]$ in the series $f(x)+p \xi+q \xi^{2}+r \xi^{3}+$ $\& c$. resulting from the expansion of $f(x+\xi)$, one can always take $\xi$ so small that whatever term be greater that the sum of all the following ones, and this also happen for all the values of $\xi$ smaller [than this].

$[\ldots]$ dans la série $f(x)+p \xi+q \xi^{2}+r \xi^{3}+\& c$. qui naît du dévelopment de $f(x+\xi)$, on peut toujours prendre $\xi$ assez petit pour qu'un terme quelconque soit plus grand que la somme de tous les termes qui le suivent; et que cela doit avoir lieu aussi pour toutes les valeurs plus petites de $\xi$.

The statement is vague. We shall try to clarify by looking at the proof.

Lagrange begins (Lagrange 1797, art. 11; 1813, art. I.3) by pointing out that $f(x)$ is "what is independent of the quantity $\xi$ " in $f(x+\xi)$, that is, "the part of $f(x+\xi)$ that remains when the quantity $\xi$ vanishes". Hence, $f(x+\xi)$ is "equal to $f(x)$ plus a quantity that must disappear if $\xi=0$ ", which could therefore be expressed as a product having a positive power of $\xi$ as a factor. But, he argues, since "in the expansion of $f(x+\xi)$ cannot enter any fractional power of $\xi$ ", it follows that this product has to be of the form $\xi P_{1}(x, \xi)$, where $P_{1}(x, \xi)$ is a function of $x$ and $\xi$ that "does not become infinite for $\xi=0$ ", so that: $f(x+\xi)=f(x)+\xi P_{1}(x, \xi)$. The same argument can be reiterated-assuming that $P_{h}(x, \xi)=P_{h}(x, 0)+\xi P_{h+1}(x, \xi)(h=1,2, \ldots)$ - to prove that: $^{97}$

$$
f(x+\xi)=\sum_{k=0}^{h} p_{k}(x) \xi^{k}+P_{h+1}(x, \xi) \xi^{h+1} \quad(h=0,1, \ldots),
$$

where $p_{0}(x)=f(x), P_{k}(x, \xi)(k=1,2, \ldots)$ are functions of $x$ and $\xi$ that do not become infinite for $\xi=0$, and $p_{k}(x)=$ $P_{k}(x, 0)$.

If the power-series expansion theorem is accepted, this argument poses no problems, since the crucial equalities $P_{h}(x, \xi)=P_{h}(x, 0)+\xi P_{h+1}(x, \xi)(h=0,1, \ldots)$, where $P_{0}(x, \xi)=f(x+\xi)$, immediately follow from this theorem, namely from the equality $P_{h}(x, \xi)=\sum_{k=h}^{\infty} p_{k}(x) \xi^{k-h}$, if $\xi$ is small enough for the power-series expansion of $f(x+\xi)$ to converge to this function.

Fraser (1987, pp. 42-43) has suggested a different interpretation, however. For him, Lagrange's proof of the equalities (79) is part of his proof of the power-series expansion theorem. This last proof should thus not be understood in the way we suggested in section 3.1. Fraser thinks that, in proving the power-series expansion theorem, Lagrange does not suppose that any function $f(x+\xi)$ has a generalized power-series expansion: having proved that "any expansion of $f(x+\xi)$ in powers of $\xi$ can contain no fractional or negative powers of $\xi$ ", he instead proves the equalities (79) and uses them to obtain the equality

$$
f(x+\xi)=\sum_{k=0}^{\infty} p_{k}(x) \xi^{k}
$$

According to Fraser, Lagrange's proof of the equalities (79) rests then on an implicit, and unjustified lemma he calls 'factor lemma':

Lemma 1 (Factor lemma). If $g(x, \xi)$ is a function of $x$ and $\xi$ such that $g(x, 0)=0$, then $g(x, \xi)=\xi^{\alpha} G(x, \xi)$, where $\alpha>0$ and $G(x, \xi)$ is a function of $x$ and $\xi$ that becomes neither infinite nor null for $\xi=0$.

\footnotetext{
${ }^{97}$ As pointed out in footnote 41 , we use ' $p_{k}(x)$ ' $(k=0,1, \ldots)$ to replace Lagrange's symbols ' $f x$ ', ' $p$ ', ' $q$ ', \&c. In the same vein, we use ' $P_{h+1}(x, \xi)$ ' $(h=0,1, \ldots)$ for Lagrange's symbols ' $P$ ', ' $Q$ ', ' $R$ ', \&c.
} 
In our view, this interpretation involves at least three difficulties.

The first concerns functions, like $x \log (1+\xi)$, having $\log (1+\xi)$ as a factor. To ensure that these functions satisfy the factor lemma-for example, that $x \log (1+\xi)=\xi^{\alpha} G(x, \xi)$, for some appropriate positive exponent $\alpha$ and function $G(x, \xi)$ - Lagrange could have appealed to two arguments. He could either have observed that $\log (1+\xi)$ can be expanded in a power-series, or he could have relied on the equality (32) in order to rewrite this factor under the form

$$
r(1+\xi)^{\frac{1}{r}}-r=\xi+\frac{1-r}{2 r} \xi^{2}+\& c .=\xi\left(1+\frac{1-r}{2 r} \xi+\& c .\right),
$$

with $r$ infinitely large. Still, as already observed above (p. 30, especially footnote 79), both in the Théorie and in the Leçons, Lagrange gets the power-series expansion of the logarithm by appealing to the power-series expansion theorem, and draws, in the Leçons, the equality (81) from this very power-series expansion. Hence, had he actually relied on the factor lemma in order to proof the power-series expansion theorem, he would have fallen into a circularity. No doubt, the power-series expansion of the logarithm could have been obtained according to different arguments than Lagrange's. But this would hardly have been done without either supposing that $\log (1+\xi)$ has a power-series expansion or relying on some geometric arguments involving the quadrature of the hyperbola.

The second difficulty concerns the very nature of a proof of equalities (79) based on the factor lemma. This proof would consists, indeed, in a reiteration of the following argument:

$$
\begin{aligned}
\text { i) } \quad P_{h}(x, \xi) & =p_{h}(x)+P_{h+1}^{*}(x, \xi) \\
& =p_{h}(x)+\xi^{\alpha} P_{h+1}(x, \xi) \\
&
\end{aligned}
$$

where $P_{h+1}^{*}(x, \xi)$ is such that $P_{h+1}^{*}(x, 0)=0, \alpha$ is positive, $P_{h+1}(x, \xi)$ becomes neither infinite nor null for $\xi=0$, and $P_{0}(x, \xi)=f(x+\xi), p_{0}(x)=f(x)$. The factor lemma would enter into this argument to justify the passage from (i) to (ii), whereas the passage from (ii) to (iii) would be justified by the previous conclusion that "any expansion of $f(x+\xi)$ in powers of $\xi$ can contain no fractional or negative powers of $\xi$ ". But, neither this lemma, nor this conclusion are suitable for justifying the assumption of $(i)$, that is, the supposition that $P_{h}(x, \xi)$ is equal to $P_{h}(x, 0)=p_{h}(x)$ plus a function of $x$ and $\xi$ that vanishes for $\xi=0$. Now, if the power-series expansion theorem is not accepted, this supposition is hard to justify, specially in the general case where $P_{h}(x, \xi)$ is not merely a function of $x+\xi$, but rather a function of $x$ and $\xi$. Hence the factor lemma would not have been the only unjustified strong assumption entering into such a proof. Lagrange would thus have derived no real advantage from proving the power-series expansion theorem this way, rather than the way we described in section 3.1.

The third difficulty relates to a simple remark: neither the equalities (79) nor their proof figure in the Leçons. Had Lagrange conceived these equalities as part of his proof of the power-series expansion theorem, he would have regarded them as a fundamental piece of his theory and would hardly have excluded them from the Leçons.

These difficulties suggest not adopting Fraser's interpretation and maintaining that Lagrange's proof of equalities (79) is not part of his proof of the power-series expansion theorem. This is because we discard Fraser's suggestion and consider that Lagrange's proof of equalities (79) relies on this very theorem, namely on the identification of the functions $P_{h}(x, \xi)$ with the series $\sum_{k=h}^{\infty} p_{k}(x) \xi^{k-h}$.

But then, what role do equalities (79) have in Lagrange's theory? If this question had no plausible answer, Fraser's interpretation would gain credence, despite its difficulties. Still, a plausible answer does exist: these equalities allow the proof of two other results.

The first consists in the following equalities (Lagrange 1797, art. 11; Lagrange 1813, art. I.3):

$$
P_{h+1}(x, \xi)=\frac{P_{h}(x, \xi)-p_{h}(x)}{\xi} \quad ; \quad p_{h+1}(x)=\left[\frac{P_{h}(x, \xi)-p_{h}(x)}{\xi}\right]_{\xi=0} \quad(h=0,1, \ldots) .
$$

These equalities, which follow immediately from equalities (79), are algorithmically equivalent to the equality involved in the modern definition of derivatives as limits of the incremental ratio. ${ }^{98}$ But they provide no operator endowed with an algebra and specific properties, and allow the (recursive) computation of the derivatives of $f(x)$ only if it is possible to

${ }^{98}$ Note however the difference between

and

$$
p_{h+1}(x)=\left[\frac{P_{h}(x, \xi)-p_{h}(x)}{\xi}\right]_{\xi=0} \text { that is, } \frac{f^{(h+1)}(x)}{(h+1) !}=\left[\frac{P_{h}(x, \xi)-\frac{f^{(h)}(x)}{h !}}{\xi}\right]_{\xi=0}
$$

$$
f^{(h+1)}(x)=\left[\frac{f^{(h)}(x+\xi)-f^{(h)}(x)}{\xi}\right]_{\xi=0} .
$$


factor out the increment $\xi$ from the numerator of the relevant ratios. ${ }^{99}$ This is not a problem for Lagrange, since for him the derivatives of $f(x)$ are not defined by these equalities, which merely provide a procedure for computing them in some cases.

The second result is just the theorem on the sufficiently small increment. To prove it (Lagrange 1797, art. 14; 1813, arts. I.6), Lagrange identifies the remainders $\xi^{h} P_{h}(x, \xi)(h=1,2 \ldots)$ with the series $\sum_{k=h}^{\infty} p_{k}(x) \xi^{k}$ and uses the equalities $P_{h-1}(x, \xi)=p_{h-1}(x)+\xi P_{h}(x, \xi)$ to argue that the products $\xi P_{h}(x, \xi)$ vanish for $\xi=0$. Hence, he implicitly uses the equality $\sum_{k=0}^{\infty} p_{k+h}(x) \xi^{k}=P_{h}(x, \xi)=\frac{P_{h-1}(x, \xi)-p_{h-1}(x)}{\xi}$, which makes it clear that he is not proving but rather assuming the convergence of $\sum_{k=0}^{\infty} p_{k}(x) \xi^{k}$ to $f(x+\xi)$ around $\xi=0$.

To be more precise, the proof runs as follows.

Since $\xi P_{h}(x, \xi)$ are functions of $\xi$ that vanish for $\xi=0$, they can be taken as the expressions of curves referred to a Cartesian system of co-ordinates $\xi, y$ passing trough the origin of this system. Moreover, unless such an origin is a singular point of these curves - which can be the case only if $x$ takes certain particular values - these curves are continuous around it $^{100}$ and thus approach the $\xi$-axis before cutting it, so as to come so close that their distances from it become smaller than any given positive quantity. Hence for any given positive quantity, it is always possible to find a positive quantity $\delta$ such that the ordinates $y=\xi P_{h}(x, \xi)$ of these curves are smaller than this quantity in absolute value, if $|\xi| \leq \delta .{ }^{101}$

It follows that, insofar as $p_{h-1}(x)$ doesn't vanish—which can only happen if $x$ takes certain particular values-it is possible to find a positive quantity $\delta$ such that $\left|\xi^{h} P_{h}(x, \xi)\right|<\left|\xi^{h-1} p_{h-1}(x)\right|$, if $|\xi| \leq \delta$. But as $\xi^{h} P_{h}(x, \xi)=\sum_{k=h}^{\infty} p_{k}(x) \xi^{k}$, this means that it is possible to find a positive quantity $\delta$ such that $\left|\xi^{h-1} p_{h-1}(x)\right|>\left|\sum_{k=h}^{\infty} p_{k}(x) \xi^{k}\right|$, if $|\xi| \leq \delta$.

This argument holds only if the upper bound $\delta$ of the absolute values of $\xi$ depends on $h$ and $x$. Lagrange's language has poor tools for specifying this necessary condition. Still, it is clear that his argument can at most prove the following result:

Theorem 8. For any function $f(x+\xi)$, any non-negative integer $h$, and any value of $x$ such that this function has a power-series expansion $\sum_{k=0}^{\infty} p_{k}(x) \xi^{k}$ and $p_{h}(x)$ does not vanish, there is a positive quantity $\delta$ such that

$$
|\xi| \leq \delta \Rightarrow\left|\xi^{h} p_{h}(x)\right|>\left|\sum_{k=h+1}^{\infty} p_{k}(x) \xi^{k}\right| .
$$

But, does Lagrange's argument actually prove this theorem? We claim it does.

The remainders $\xi P_{h}(x, \xi)$ are understood in this argument as geometric quantities, namely ordinates of curves. Lagrange thus seems to follow an old tradition in conceiving segments as universal magnitudes, $i$. e. magnitudes capable of representing quantities of any kind. This allows him to reduce what he takes to be a property of any function to a property of one-piece curves. Insofar as the former is a property of any function, there is no need to justify the fact that the functions $\xi P_{h}(x, \xi)$ have it. But insofar as one-piece curves can be opposed to multi-piece curves, this property can be viewed as a special one.

Thus, by introducing curves alongside functions, Lagrange manages to transpose a property of certain curves-namely one-piece curves- to all functions. Though, mutatis mutandis, this property is what we call 'continuity' (following

\footnotetext{
${ }^{99}$ It follows that these equalities lead to the determination of the derivatives of $f(x)$ only for algebraic functions, since in case of transcendental ones, $\xi$ can be factored out from the differences $P_{h}(x, \xi)-p_{h}(x)$ only replacing $P_{h}(x, \xi)$ with their power series expansions. Moreover-as Lagrange remarks, considering the example of the function $f(x)=\sqrt{x}$ (Lagrange 1797, art. 13; 1813, art. I.5) — even in the case of algebraic functions can sometimes be "more expeditious" to compute the derivatives of $f(x)$ by relying on the power-series expansion of $f(x+\xi)$.

${ }^{100}$ Lagrange writes: "after this point". He thus seems to take $\xi$ to be non-negative. The generalization of his argument to the case of any $\xi$ is easy, however.

${ }^{101}$ To avoid misunderstandings we quote Lagrange:

[...] consider the curve of which $\xi$ would be the abscissa and one of the $[\ldots]$ functions $\left[\xi P_{h}(x, \xi)\right]$ the ordinate. This curve will cut the axis in the origin of the abscissas, and, unless this point is singular, which can only happen for some particular values of $x[\ldots]$, the path of the curve will necessarily be continuous after this point. Hence, this curve will gradually come near the axis before cutting it, to the effect that it will come to be out of it for a quantity smaller than any given quantity. Thus, one could always find an abscissa corresponding to an ordinate smaller that any given quantity, and then any value of $x$ smaller [than this one] will also correspond to an ordinate smaller than the given quantity.

[... en considérant la courbe dont $\xi$ serait l'abscisse, et l'une de[s][...] fonctions $\left[\xi P_{h}(x, \xi)\right]$ l'ordonnée, cette courbe coupera l'axe à l'origine des abscisses; et, à moins que ce point ne soit un point singulier, ce qui ne peut avoir lieu que pour des valeurs particulières de $x$ [...], le cours de la courbe sera nécessairement continu depuis ce point; donc elle s'approchera peu à peu de l'axe avant de le couper, et s'en approchera par conséquent d'une quantité moindre qu'aucune quantité donnée; de sorte qu'on pourra toujours trouver une abscisse $\xi$ correspondant à une ordonnée moindre qu'une quantité donnée; et alors toute valeur plus petite de $\xi$ répondra aussi à des ordonnées moindres que la quantité donnée. 
Cauchy), for Lagrange it is not a property of a class of functions, but rather a property of one-piece curves reflected by a property of any function: its introduction obey only to a descriptive purpose and not to the purpose of characterizing an appropriate class of functions (Grabiner 1990, p. 143; 1981a, p. 95).

Furthermore, insofar as what is described is the behavior of a one-piece curve in the neighborhood of a point where it is defined, this property does not concern this point but a neighborhood of it. In other words, Lagrange is not saying that a function $f(\xi)$ is continuous at some point $\xi=a$ if (and only if), for any given positive quantity $\varepsilon$, there is a positive quantity $\delta$ such that $|f(\xi)-f(a)|<\varepsilon$, if $|\xi-a| \leq \delta$. He is rather arguing that any function $f(\xi)$ which is defined for a certain value $\xi=a$-which is not a singular one-is such that, for any given positive quantity $\varepsilon$, there is a positive quantity $\delta$ such that $f(\xi)$ is defined and $|f(\xi)-f(a)|<\varepsilon$, if $|\xi-a| \leq \delta .^{102}$

Once this last statement is accepted, Lagrange's argument seems to be thoroughly correct and actually to prove theorem 8. ${ }^{103}$ As we shall see in the next section, this last theorem is, however, too weak for correctly playing the role that, in the first edition of the Théorie, Lagrange assigns to the theorem of the sufficient small increment within his proof of the remainder theorem.

5.2.2. Two lemmas for the remainder theorem: presuppositions of uniformity. The theorem of the sufficient small increment enters into this proof as a premise for proving a lemma from which the remainder theorem is then deduced. In the second edition of the Théorie, Lagrange proves the same lemma by relying on the equality $f(x+\xi)=f(x)+\xi P_{1}(x, \xi)$ rather than on the former theorem. ${ }^{104}$ Still, the argument in the second edition presents difficulties similar to those presented by that of the first edition.

In the Leçons, this lemma is replaced by another lemma whose proof depends neither on the theorem of the sufficiently small increment nor on the equality $f(x+\xi)=f(x)+\xi P_{1}(x, \xi)$, or, more generally, on the equalities (79). This proof is also flawed, however, and for reasons similar to those that undermine the proofs of the lemma of the Théorie.

It therefore seems convenient to consider both lemmas before turning to the proofs of the remainder theorem.

\subsubsection{The LEMMA OF THE Théorie}

The lemma of the Théorie is the following (Lagrange 1797, art. 48; 1813, art. I.38; the proofs are given in the same articles):

Lemma 2 (Lemma of the Théorie). If a prime function of $x$ like $f^{\prime}(x)$ is always positive for any value of $x$ from $x=a$ up to $x=b$, supposing that $b$ be $>a$, the difference of the primitive functions corresponding to these two values of $x$, i.e. $f(b)-f(a)$, will necessarily be a positive quantity.

Si une function prime de $x$, telle que $f^{\prime}(x)$, est toujours positive pour toutes les valeurs de $x$, depuis $x=a$ jusqu'à $x=b$, $b$ étant $>a$, la différence des fonctions primitives qui répondent à ces deux valeurs de $x$, savoir, $f(b)-f(a)$, sera nécessairement une quantité positive.

Once more, the statement is vague. In to-day's French, 'positif' often means 'non-negative'. But it is unclear whether Lagrange takes 'positif' to mean this or rather 'greater than zero' or 'positive', in current English. Though this is a crucial detail if the function $f(x)$ is conceived as an algebraic quantity rather than as the expression of a curve, Lagrange seems uninterested in it. Because he seeks generality, one could argue. But generality could hardly be a reason for licensing conflating the conditions $\alpha>0$ and $\alpha \geq 0$, when an argument or result turns on their distinction. Hence, we find hard to account for Lagrange's attitude. We shall then reconstruct his arguments and results by interpreting them as strongly and charitably as possible.

Lagrange's proof can be divided into two parts (a reconstruction of this proof is provided by Grabiner 1990, pp. 219221).

The first part is different in the two editions of the Théorie.

In the first edition, he relies on the theorem of the sufficiently small increment to state that $\xi$ can be taken such that the term $\xi f^{\prime}(x)$ in the power-series expansion of $f(x+\xi)$ be greater (in absolute value) than $\sum_{k=2}^{\infty} \frac{f^{(k)}(x)}{k !} \xi^{k}$. Next, he takes

\footnotetext{
${ }^{102}$ Despite such an essential difference, the way Lagrange formulates his property certainly prefigures Cauchy's definition and even the Weierstrassian $\varepsilon-\delta$ interpretation of it. This is not an isolated fact: in order to prove the remainder theorem, Lagrange uses several arguments that are amazingly close to Cauchy's and Weierstrass' techniques. The point has been often made (for example by Grabiner 1981a, pp. 56-76, and Grabiner 1990, pp. 171-214), and we shall not dwell on it. We shall confine ourselves instead to a reconstruction of these arguments within the context of Lagrange's theory, which is quite different from Cauchy's and Weiertrass' versions of the calculus (Fraser 1987, p. 52).

${ }^{103}$ To prove theorem 8 , it is necessary to identify the absolute value of $p_{h-1}(x)(h=1,2, \ldots)$ with the positive quantity $\varepsilon$. If this identification is left out, Lagrange's argument provides a proof for another theorem that he does not state explicitly, and that could be formulated as follows: for any function $f(x+\xi)$, any natural number $h$, any value of $x$ such that this function has a power-series expansion $\sum_{k=0}^{\infty} p_{k}(x) \xi^{k}$, and any positive quantity $\varepsilon$, there is a positive value $\delta$ such that $|\xi| \leq \delta \Rightarrow\left|\sum_{k=h}^{\infty} p_{k}(x) \xi^{k}\right|<\varepsilon$.

${ }^{104}$ It follows that — according to our understanding — the only effective role that the equalities (79) for $h>0$ have in the second edition of the Théorie is that of allowing proving the equalities (82).
} 
advantage of this possibility to conclude that, if $f^{\prime}(x)$ is "positif", $\xi$ can be taken small enough, while remaining "positif", for $f(x+\xi)-f(x)=\sum_{k=1}^{\infty} \frac{f^{(k)}(x)}{k !} \xi^{k}$ to be "positif", too.

In the second edition, he relies on the equality $f(x+\xi)=f(x)+\xi P_{1}(x, \xi)$ and remarks that, insofar as $P_{1}(x, 0)=$ $p_{1}(x)=f^{\prime}(x)$, it follows that, if $f^{\prime}(x)$ is "positif", then, "from $\xi=0$ up to a certain value of $\xi$, which one could take as small as one wishes [depuis $\xi=0$ jusqu'à une certaine valeur de $\xi$, qu'on pourra prendre aussi petite qu'on voudra]", $P_{1}(x, \xi)$ has to be "positif" too. He then uses this to conclude that, if $f^{\prime}(x)$ is "positif", $\xi$ can be taken small enough, while remaining "positif", for $f(x+\xi)-f(x)$ to be "positif" too.

Let us consider these two arguments separately, by supposing - to begin with-that $f^{\prime}(x)>0$.

For the former argument to hold, it is enough to take the theorem of the sufficiently small increment to be equivalent to theorem 8. If $f^{\prime}(x)>0$, it is indeed enough to apply this last theorem for $h=1$, together with theorem 4 , to conclude that there is a positive quantity $\delta$ such that

$$
0<\xi \leq \delta \Rightarrow \sum_{k=1}^{\infty} \frac{f^{(k)}(x)}{k !} \xi^{k}>0,
$$

from which

$$
0<\xi \leq \delta \Rightarrow f(x+\xi)-f(x)>0
$$

follows, if $\sum_{k=1}^{\infty} \frac{f^{(k)}(x)}{k !} \xi^{k}$ is replaced by $f(x+\xi)-f(x)$.

For the latter argument to hold, it is necessary for the function $P_{1}(x, \xi)$ to possess the same property that Lagrange assigns to all the functions $\xi P_{h}(x, \xi)(h=1,2, \ldots)$ in his proof of the theorem of the sufficiently small increment. If $f^{\prime}(x)=P_{1}(x, 0)>0$, it follows that $P_{1}(x, \xi)=f(x+\xi)-f(x)>0$ in a right neighborhood of $\xi=0$, and there is thus a positive quantity $\delta$ such that the implication (85) holds. The assumption that $P_{1}(x, 0)=f^{\prime}(x)$ is not trivial, however. Either it is licensed by taking the first derivative of a function $f(x)$ to coincide by definition with $\left[\frac{f(x+\xi)-f(x)}{\xi}\right] \xi=0$, which is incompatible with Lagrange's theory. Or it depends on the assumption that $f(x+\xi)$ has an expansion whose two first terms are $f(x)$ and $p_{0}(x) \xi$. But if this is assumed, Lagrange's latter argument-which is, at first glance, independent of the assumption that $f(x+\xi)$ has a power-series expansion-reduces to a merely linguistic variant of the former.

The two arguments also resemble each other if it supposed that $f^{\prime}(x)=0$. Neither of them can be used to prove implication (85), or even the weaker implication $0<\xi \leq \delta \Rightarrow f(x+\xi)-f(x) \geq 0$. This means that either Lagrange excludes the case $f^{\prime}(x)=0$ from the range of his lemma - that is, he takes the adjective 'positif' applied to both $f^{\prime}(x)$ and $f(x+\xi)-f(x)$ to mean 'greater than zero'-or he supposes that $f^{\prime}(x)=0$ only if $f(x)$ reduces to a constant (so that $f(x+\xi)-f(x)=0)$, i. $e$. he excludes the values of $x$ for which $f^{\prime}(x)=0$, for any function that does not reduce to a constant.

These are not the only difficulties of Lagrange's proofs of the lemma of the Théorie. A much more relevant one concerns the second part of these proofs, which is the same in the two editions of the Théorie. To see this difficulty some preliminary remarks are necessary.

In both foregoing arguments providing the first part of Lagrange's proof, as well as in Lagrange's proof of the theorem of the sufficiently small increment, the functions $\xi P_{h}(x, \xi)(h=1,2, \ldots)$ are considered as functions of the variable $\xi$ alone. The variable $x$ is simply supposed to be such that the value $\xi=0$ is not a singular value of these functions and the function $f(x+\xi)$ has a power-series expansion. Lagrange merely supposes that for any function $g(\xi)$ defined in $\xi=0$ for which this point is not singular, there is a neighborhood of this same point where $|g(\xi)-g(0)|$ is smaller than any given positive quantity $\varepsilon$. There is thus no room for any ambiguity concerning the uniformity of this property. Indeed, if the variable $x$ is not considered, this neighborhood can only depend on $\varepsilon$.

When the functions $\xi P_{h}(x, \xi)$ are instead considered as functions of two variables $x$ and $\xi$, the situation changes. Since, it is one thing to assert that:

- [Con.1] A function $g(x, \xi)$ which is defined for a certain value $\xi=a$ whenever $x$ belongs to a certain interval $I_{x}$, is such that, for any given positive quantity $\varepsilon$ and for any $x$ in $I_{x}$, there is a positive quantity $\delta$ such that, if $|\xi-a| \leq \delta$, then $g(x, \xi)$ is defined and $|g(x, \xi)-g(x, a)|<\varepsilon$.

Quite another to assert that:

- [Con.2] A function $g(x, \xi)$ which is defined for a certain value $\xi=a$ whenever $x$ belongs to a certain interval $I_{x}$, is such that, for any given positive quantity $\varepsilon$, there is a positive quantity $\delta$ such that, for any $x$ in $I_{x}$, if $|\xi-a| \leq \delta$, then $g(x, \xi)$ is defined and $|g(x, \xi)-g(x, a)|<\varepsilon$.

In the latter case, the property possessed by $g(x, \xi)$ is uniform, since $\delta=\delta(\xi)$; in the former case, it is not, since $\delta=\delta(x, \xi)$.

Suppose now that the functions $\xi P_{h}(x, \xi)$ be understood, both in Lagrange's proof of the theorem of the sufficiently small increment and in the two foregoing arguments, as functions of the two variables of $x$ and $\xi$. Nothing would ensure 
that these functions enjoy the property [Con.2]. The property that one would be licensed to ascribe to them would rather be [Con.1]. In this way, one would obtain linguistic variants of Lagrange's proof and arguments, suitable for proving theorem 8 and implication (85) respectively, under the assumption that $\delta$ depends on $x$ (and that $f^{\prime}(x)>0$ ).

But for the second part of Lagrange's proofs of the lemma of the Théorie to be sound, the implication (85) has to be enter into it under the assumption that $\delta$ be independent of $x$. And this assumption would be permitted only if it were possible to assign to the functions $\xi P_{h}(x, \xi)$ the property [Con.2].

Look at the details. Lagrange takes $\xi$ to be equal to $\frac{b-a}{n+1}$. If $b>a$, for any positive quantity $\delta$ there is a non-negative integer $n$ such that $\frac{b-a}{n+1} \leq \delta$. By relying on the implication (85), he concludes then that if all the derivatives $f^{\prime}\left(a+k \frac{b-a}{n+1}\right)$ $(k=0,1, \ldots, n)$ are "positif", also the differences

$$
f\left(a+(k+1) \frac{b-a}{n+1}\right)-f\left(a+k \frac{b-a}{n+1}\right)
$$

and their sum

$$
\sum_{k=0}^{n}\left[f\left(a+(k+1) \frac{b-a}{n+1}\right)-f\left(a+k \frac{b-a}{n+1}\right)\right]=f(b)-f(a)
$$

are "positif". Next, he draws from this that if $f^{\prime}(x)$ is "positive" whenever $x$ takes all possible values "from $x=a$ up to $x=b$ [depuis $x=$ a jusqu' $\grave{a} x=b]$ ", the difference $f(b)-f(a)$ is "positif" too.

This argument is clearly based on the assumption that the functions $\xi P_{h}(x, \xi)$ have property [Con.2], which is an unproved and hardly acceptable assumption of uniformity. This makes this argument, and then Lagrange's proof of the lemma of the Théorie flawed.

It is implausible to argue that Lagrange saw the problem and deliberately accepted to make this strong assumption. It is likelier that he could not tell the properties [Con.1] and [Con.2] apart.

Having looked at this crucial difficulty of Lagrange's proof, let us now try to elucidate the exact content of the lemma.

Supposing that $x$ take all the possible values "from $x=a$ up to $x=b$ ", Lagrange seems to refer unambiguously to the closed interval $[a, b]$. Lagrange's argument requires, however, neither that $f^{\prime}(b)>0$ nor that $f^{\prime}(b) \geq 0$. One could thus concede that the antecedent of the lemma reduces to the condition that $f^{\prime}(x)$ is defined and is "positif" in $[a, b)$, provided of course that $f(x)$ is defined for $x=b$.

But how is 'positif' to be understood here? If we took this to mean 'greater than zero,' or we took Lagrange to assume that condition $f^{\prime}(x)=0$ may hold only if $f(x)$ reduces to a constant, his proof would pose no other problems. However, as we shall see later, in proving the remainder theorem in the Théorie, Lagrange applies his lemma under the condition that $f^{\prime}(x) \geq 0$ and $f(x)$ is not constant. Is there a way to understand this lemma in such a way that it could be applied under this condition? As a matter of fact, there is one, but it depends on assumptions that are far from trivial. Let us consider it.

It is clear that if $f^{\prime}(a) \neq 0$, and the interval $[a, b)$ contains only a finite number of isolated values of $x$ for which $f^{\prime}(x)=$ 0 , it is always possible to chose $n$ so that these values do not include anyone of the values $a+k \frac{b-a}{n+1}(k=0,1, \ldots, n) .{ }^{105}$ In this case, equality (85) applies and—apart for the unjustified assumption of uniformity_Lagrange's argument holds.

The requirement that $f^{\prime}(a) \neq 0$ is too restrictive, however, since, in proving the remainder theorem in the Théorie, Lagrange applies his lemma to two functions whose derivatives vanish for the right bound of the relevant interval. To justify Lagrange's proof, it would thus be necessary to eliminate this requirement from the previous statement. Fortunately, this can be done. The reason is the following.

Assume that $f^{\prime}(a)=0$ and that the interval $[a, b)$ contains only a finite number of isolated values of $x$ for which $f^{\prime}(x)=0$. For any positive value $\delta$, it is then possible to take a value $\bar{a}$ of $x$ such that $0<a-\bar{a}<\delta$ and $f^{\prime}(\bar{a}) \neq 0$. Now Lagrange clearly supposes that if $f^{\prime}(x)$ is defined for any $x$ in $[a, b)$, then $f(x)$ is defined there as well, and is thus such that, for any positive quantity $\varepsilon$, there is a positive quantity $\delta$ such that $|f(a)-f(\bar{a})|<\varepsilon$ if $|a-\bar{a}|<\delta$. From this it follows that for any positive quantity $\varepsilon$ it is possible to take a value $\bar{a}$ of $x$ such that the difference $f(b)-f(\bar{a})$ differs from the difference $f(b)-f(a)$ for a quantity that is smaller than $\varepsilon$. This is enough to eliminate the condition $f^{\prime}(a) \neq 0$, and to conclude that, if the interval $[a, b)$ contains only a finite number of isolated values of $x$ for which $f^{\prime}(x)=0$, the equality (85) applies even if $f^{\prime}(a)=0$.

All these considerations bring us to suggest the following reformulation of the lemma of the Théorie: ${ }^{106}$

Lemma 3. If $f(x)$ is not constant and its first derivative $f^{\prime}(x)$ is defined and is non-negative for any $x$ in an interval $[a, b)(a<b)$ that does not contain infinitely many values of $x$ for which $f^{\prime}(x)$ vanishes and is such that $f(x)$ is defined for $x=b$, then the difference $f(b)-f(a)$ is non-negative, too.

\footnotetext{
${ }^{105}$ In fact, it suffices that among the isolated values of $x$ belonging to $[a, b)$ for which $f^{\prime}(x)=0$ there are not infinitely many points $z$ such that $z-a$ is rational.

${ }^{106}$ Ovaert (1976, pp. 187-188), and Dugac (2003, p. 74) also take $f^{\prime}(x)$ and $f(b)-f(a)$ to be non-negative, but Ovaert takes the interval from $a$ to $b$ to be closed.
} 


\subsubsection{THE LEMMA OF THE Leçons}

The lemma of the Leçons is the following (Lagrange 1801, p. 66; 1806, p. 86; we quote from the second edition; in the first one, Lagrange considers only a right neighborhood of the origin):

Lemma 4 (Lemma of the Leçons). A function that vanishes when the variable does will necessarily have, insofar as the variable increases positively, some finite values having the same sign as those of its derivative function, or the opposite sign, if the variable increases negatively, insofar as the values of the derivative function will conserve the same sign and do not become infinite.

Une fonction qui est nulle lorsque la variable est nulle, aura nécessairement, pendant que la variable croîtra positivement, des valeurs finies et de même signe que celles de sa fonction dérivée, ou de signe opposé si la variable croît négativement, tant que les valeurs de la fonction dérivée conserveront le même signe, et ne deviendront pas infinies.

Also in this case, the statement is vague. To begin with, the terms 'même signe' and 'signe opposé' are not clear: for two functions to have the same sign, do they both have to be greater or smaller than zero? Or is it enough for them both to be non-negative or non-positive? Furthermore, it is not clear on what intervals the variable is supposed to be increasing or decreasing, or the function to have the same sign as its first derivative or the opposite sign.

Although these details are crucial if the function $f(x)$ is to be viewed as an algebraic quantity rather than as the expression of a curve, Lagrange ignores them, just as he does in the Théorie for analogous details. ${ }^{107}$ Here again, we can only confine ourselves to a reconstruction of his arguments and results, interpreting them as strongly and charitably as possible.

To prove his lemma (Lagrange 1801, pp. 67-69; 1806, pp. 89-92), ${ }^{108}$ Lagrange begins by remarking that, if the first derivative $f^{\prime}(x)$ of a function $f(x)$ is not infinite for a certain value of $x$, then, for this same value of $x$, the first two terms of the power-series expansion of $f(x+\xi)$ are certainly "exact". Hence, he continues, even if the whole power-series expansion of $f(x+\xi)$ is not defined for this value of $x$, if $f^{\prime}(x)$ is not infinite for such a value, $f(x+\xi)$ has, for this same value, an expansion that begins with $f(x)+f^{\prime}(x) \xi$, and whose all other terms include powers of $\xi$ whose exponent is greater that 1 , to the effect that:

$$
f(x+\xi)=f(x)+\xi\left[f^{\prime}(x)+V(x, \xi)\right]
$$

where $V(x, \xi)$ is a function of $x$ and $\xi$ such that $V(x, 0)=0$.

These are strange claims within the context of Lagrange's theory. Since, according to the basic principles of this theory, $f^{\prime}(x)$ is not defined for a certain value of $x$ if the two first terms of the power-series expansion of $f(x+\xi)$ are not "exact" for this value. It follows that, according to these principles, claiming that $f^{\prime}(x)$ is not infinite for a certain value of $x$ is the same as claiming that the first two terms of the power-series expansion of $f(x+\xi)$ are "exact" for this value. For the former claim not to implicitly contain the latter, the function $f^{\prime}(x)$ has to be defined independently of the first two terms of the power-series expansion of $f(x+\xi)$, for instance as the result of an appropriate algorithm to be applied to $f(x)$, or as the limit (or the value) of the ratio $\frac{f(x+\xi)-f(x)}{\xi}$ when $\xi$ tends (or is equal) to 0 . Hence, the way as Lagrange expresses himself here seems to implicitly contradict the principles of his theory, and tacitly imply that $f^{\prime}(x)$ is not defined according to them.

This could be viewed as no more than a slip of the pen. But then, the starting point of Lagrange's proof cannot be taken to be the supposition that the first derivative $f^{\prime}(x)$ of a function $f(x)$ is not infinite (as it appears at first glance), but rather the assumption that equality (88) holds.

Be that as it may, once taken this equality for granted, Lagrange applies to the function $V(x, \xi)$ a non-geometric version of the argument he applies to the functions $y=\xi P_{h}(x, \xi)(h=1,2, \ldots)$ in his proof of the theorem of the sufficiently small increment: if $\xi$ increases imperceptibly in absolute value starting from 0 , the absolute value of $V(x, \xi)$ will increase just

${ }^{107}$ This does not prevent Lagrange from remarking (Lagrange 1801, pp. 69-70; 1806, pp. 92-93) that within the differential calculus a similar but incorrect statement can be proved. He argues that, according to this calculus, if $\frac{d y}{d x}$ is always "positif" in an appropriate interval, $\int_{0}^{z} \frac{d y}{d x} d x$ should be too, since this is defined as the sum of the ratios $\frac{d y}{d x}$. Then he remarks that this is contradicted by the function $y=\frac{x}{a(a-x)}$, since $\frac{d y}{d x}=\frac{1}{(a-x)^{2}}$ is always "positf", whereas $\int_{0}^{z} \frac{d y}{d x} d x=\frac{z}{a(a-z)}$ becomes "negatif" when $z$ is greater than $a$. This is a strange claim, however: if $z \geq a$, according to the principles of the differential calculus correctly understood, $\int_{0}^{z} \frac{d x}{(a-x)^{2}}$ is not defined, so that the example of the function $y=\frac{x}{a(a-x)}$ contradicts neither the previous statement nor these principles.

${ }^{108}$ A reconstruction of Lagrange's proof is given in Grabiner (1981a), pp. 122-126, and (1990), pp. 181-186. A simplified version of it-with the same shortcomings-is offered by Lacroix (1810-1819), vol. I, p. 382. 
as imperceptibly from $V(x, 0)=0$, so that, for any positive quantity $\varepsilon$, there is a positive quantity $\delta$ such that $^{109}$

$$
|\xi| \leq \delta \Rightarrow|V(x, \xi)|<\varepsilon
$$

Everything said in relation to this last argument holds here too. Lagrange is merely describing the behavior of the function $V(x, \xi)$, understood as a function of the only variable $\xi$, in the neighborhood of the origin (where it is supposed to be defined). Still, if this functions is taken to be a function of $x$ and $\xi$, this description amounts to taking for granted that it has the property [Con.1], but not necessarily the property [Con.2].

Aside from this, it is far from clear whether Lagrange identifies the function $V(x, \xi)$ with a generalized power series or with the ratio $\frac{f(x+\xi)-f(x)-\xi f^{\prime}(x)}{\xi}$. As $p_{1}(x)=f^{\prime}(x)$, from the comparison of the equality (88) with the second of the equalities (79), one gets that $V(x, \xi)=\xi P_{2}(x, \xi)$. However, whereas in deriving these last equalities, Lagrange seems to assume that the function $f(x+\xi)$ has a power-series expansion and to identify the function $P_{2}(x, \xi)$ with the power series $\sum_{k=0}^{\infty} p_{k+2}(x) \xi^{k}$ - which is supposed to be convergent around $\xi=0$ - his language is now elusive enough to suggest that he wants to suppose a priori neither that $x$ is such that $f(x+\xi)$ has a power-series expansion, nor that its generalized power-series expansion $f(x)+\xi f^{\prime}(x)+\sum_{k=2}^{\infty} p_{k}(x) \xi \alpha_{k}$ converges to this function around $\xi=0$ and $V(x, \xi)=\sum_{k=2}^{\infty} p_{k}(x) \xi \alpha_{k}$. But if this is not assumed, nothing allows us to conclude that $V(x, 0)=0$.

The only way to avoid a petitio principii thus is by openly defining the derivative $f^{\prime}(x)$ of a function $f(x)$ through the equality (88), provided that $V(x, 0)=0$. According to such a definition, the first derivative of $f(x)$ would be the function $g(x)$ which, around $\xi=0$, satisfies the condition

$$
f(x+\xi)=f(x)+g(x) \xi+V(x, \xi) \xi
$$

where $V(x, 0)=0$.

This resembles to the modern definition of the derivative of $f(x)$ as the function $g(x)$ such that, around $\xi=0:{ }^{110}$

$$
f(x+\xi)-f(x)=\xi g(x)+\xi V(x, \xi) \quad\left[\text { where } \lim _{\xi \rightarrow 0} V(x, \xi)=0\right]
$$

But, even if many of Lagrange's arguments could be rephrased (and perhaps clarified) by adopting such a definition, ascribing it to him does not seem appropriate (Grabiner 1981a, pp. 116 and 120; 1990, pp. 157 and 182). It thus seems that Lagrange-despite his proof of the power-series expansion theorem-tries to free his proof of the lemma of the Leçons from the assumption that any function $f(x+\xi)$ has, for any $x$ for which $f(x)$ is not infinite, a generalized power-series expansion that converges to such a function around $\xi=0$. But he fails.

However the functions $f^{\prime}(x)$ and $V(x, \xi)$ are defined and conceived, after having stated the equality (88) and having argued that, for any positive quantity $\varepsilon$, there is a positive value $\delta$ for which the implication (89) holds, Lagrange's proof proceeds according to an argument that closely resembles the argument used in proving the lemma of the Théorie. It can be reconstructed as follows.

From these premises, Lagrange infers that for any positive quantity $\varepsilon$, there is a positive quantity $\delta$ such that:

$$
|\xi| \leq \delta \Rightarrow\left\{\begin{array}{lll}
\xi\left[f^{\prime}(x)-\varepsilon\right]<f(x+\xi)-f(x)<\xi\left[f^{\prime}(x)+\varepsilon\right] & \text { if } & \xi>0 \\
\xi\left[f^{\prime}(x)+\varepsilon\right]<f(x+\xi)-f(x)<\xi\left[f^{\prime}(x)-\varepsilon\right] & \text { if } & \xi<0
\end{array}\right.
$$

He then argues that, as these implications depend on $f^{\prime}(x)$ not being infinite, one can replace $x$ with $x+k \xi(k=1,2, \ldots, n-$ $1)$, so that, if $n$ is such that the derivatives $f^{\prime}(x+k \xi)(k=0,1, \ldots, n-1)$ are not infinite, for any positive quantity $\varepsilon$, there

${ }^{109}$ On this argument, see Grabiner (1974), p. 363. Here is what Lagrange writes:

[...] since $V$ vanishes when $\xi$ does, it is clear that, letting $\xi$ insensibly increase gradually after zero, the value of $V$ will also insensibly increase after zero, either positively or negatively, up to a certain point, after which it will be able to decrease. Hence, one will always be able to assign to $\xi$ such a value that the corresponding value of $V$, be, independently of the sign, smaller than a given quantity, and that the values of $V$ be smaller [than this quantity] also for the values of $\xi$ smaller than this value.

$[\ldots]$ puisque $V$ devient nul lorsque $\xi$ devient nul, il est clair qu'en faisant croitre $\xi$ par degrés insensibles depuis zéro, la valeur de $V$ croîtra aussi insensiblement depuis zéro, soit en plus ou en moins, jusqu'à un certain point, après quoi elle pourra diminuer; que par conséquent on pourra toujours donner à $\xi$ une valeur telle que la valeur correspondante de $V$, abstraction faire du signe, soit moindre qu'une quantité donnée, et que par les valeurs moindres de $\xi$ la valeur de $V$ soit aussi moindre. (Lagrange 1801, p. 67; 1806, p. 90.)

${ }^{110} \mathrm{~A}$ generalization of this definition to any order $h$ leads, in fact, to a definition resembling that of the Peano derivatives (Peano 1891$)$ : a real function $f(x)$ is $h$ times Peano differentiable at $x=x_{0}$ if there are $h+1$ real numbers $a_{k}(k=0,1, \ldots, h)$ such that, in an appropriate neighborhood of $x_{0}$,

$$
f(x)=\sum_{k=0}^{h}\left[\frac{a_{k}}{k !}\left(x-x_{0}\right)^{k}\right]+F_{h}(x)\left(x-x_{0}\right)^{k} \quad\left[\text { where } \lim _{x \rightarrow x_{0}} F_{h}(x)=0\right] .
$$

The real numbers $a_{k}$ are then the Peano derivatives of order $h$ at $x=x_{0}$. 
is a positive value $\delta$ such that, if $|\xi| \leq \delta$, all the inequalities

$$
\begin{array}{lll}
\xi\left[f^{\prime}(x+k \xi)-\varepsilon\right]<f(x+(k+1) \xi)-f(x+k \xi)<\xi\left[f^{\prime}(x+k \xi)+\varepsilon\right] & \text { if } \quad \xi>0 \\
\xi\left[f^{\prime}(x+k \xi)+\varepsilon\right]<f(x+(k+1) \xi)-f(x+k \xi)<\xi\left[f^{\prime}(x+k \xi)-\varepsilon\right] & \text { if } \quad \xi<0
\end{array}
$$

(where the derivatives $f^{\prime}(x+k \xi)$ are of course taken with respect to $x$ ), holds at the same time. At this point, Lagrange introduces the hypothesis that all the derivatives $f^{\prime}(x+k \xi)$ "have the same sign" "111 and adds up the inequalities, so as to get

$$
\begin{array}{ll}
\xi \sum_{k=0}^{n-1}\left[f^{\prime}(x+k \xi)\right]-n \xi \varepsilon<f(x+n \xi)-f(x)<\xi \sum_{k=0}^{n-1}\left[f^{\prime}(x+k \xi)\right]+n \xi \varepsilon & \text { if } \quad \xi>0 \\
\xi \sum_{k=0}^{n-1}\left[f^{\prime}(x+k \xi)\right]+n \xi \varepsilon<f(x+n \xi)-f(x)<\xi \sum_{k=0}^{n-1}\left[f^{\prime}(x+k \xi)\right]-n \xi \varepsilon & \text { if } \quad \xi<0 .
\end{array}
$$

Then, he takes it for granted that $\varepsilon$ can be taken smaller than $\frac{\left|\sum_{k=0}^{n-1}\left[f^{\prime}(x+k \xi)\right]\right|}{n}$, and concludes that there is a positive value $\delta$ such that:

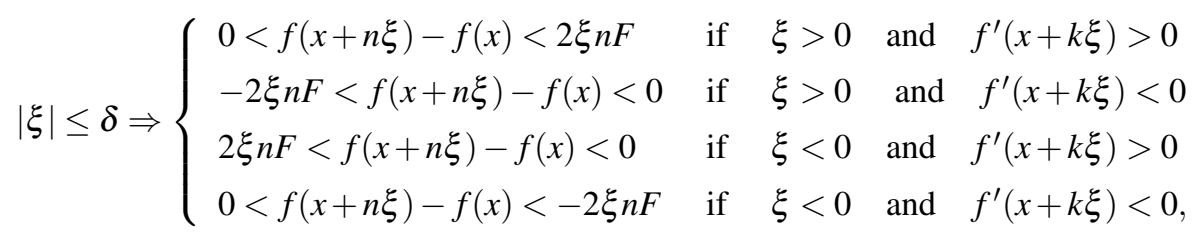

where $F=\max _{k=0, \ldots, n-1}\left|f^{\prime}(x+k \xi)\right|$. Finally, he remarks that, as $n$ increases when $\xi$ diminishes, the product $\xi n$ in the inequalities (95) can be identified with any variable $z$, so that the difference $f(x+n \xi)-f(x)$ can be regarded as a function of $z$ that vanishes for $z=0$ and whose first derivative is the same as the first derivative of $f(x+n \xi)$ (since $\left.[f(x+z)-f(x)]_{z}^{\prime}=f_{z}^{\prime}(x+z)=f_{x}^{\prime}(x+z)=f_{x}^{\prime}(x+n \xi)\right)$.

The argument is flawed. In deriving the implications (92), Lagrange seems to treat the value of $x$ as fixed. If it were, $\delta$ would depend only on $\varepsilon$. But the same argument applies for any fixed value of $x$. Hence, if a (finite) number $n$ of values of $x$ were fixed in advance (whatever $n$ may be), for any positive quantity $\varepsilon$, there would be a positive quantity $\delta$ such that all the implications like (92) relative to these values of $x$ would hold at the same time, if $|\xi| \leq \delta$. But in Lagrange's argument, the $n$ values of $x$ are not fixed in advance but depend on the increment $\xi$, which is in turn supposed to be such that $|\xi| \leq \delta$. So the argument holds only if the value $\delta$ occurring in the implications (92) is independent of $x$, that is, if the function $V(x, \xi)$ has the property [Con.2] (rather than merely the property [Con.1]).

Also in this case, Lagrange's argument is therefore actually subject to a uniformity assumption. Moreover, this assumption closely resembles the ones that figure in the second part of Lagrange's proofs of the lemma of the Théorie. ${ }^{112}$

This same assumption of uniformity is also needed to justify the identification of the product $\xi_{n}$ with any variable $z$ and the interpretation of the difference $f(x+n \xi)-f(x)$ as a function of $z$.

But it is still not enough to justify Lagrange's appeal to the condition $\varepsilon<\frac{\left|\sum_{k=0}^{n-1}\left[f^{\prime}(x+k \xi)\right]\right|}{n}$. For $\frac{\left|\sum_{k=0}^{n-1}\left[f^{\prime}(x+k \xi)\right]\right|}{n}$ depends on $\xi$, which is supposed to be smaller, in absolute value, than the positive quantity $\delta$, which in turn depends on the choice of $\varepsilon$. This was first pointed out by Bolzano (1817, pp. 19-20), who saw a gap in Lagrange's proof of the lemma of the Leçons that could only be filled using the theorem of intermediate values (Sebestik 1964, pp. 142-143). In fact, this theorem is implicitly used by Lagrange in his proof of the remainder theorem, both in the Théorie and in the Leçons (see pp. 53 and 54 , below). But he does not use it to prove his lemma.

Let us now return to the conclusion of Lagrange's argument. Let $\varphi(z)$ be the difference $f(x+n \xi)-f(x)$ regarded as a function of $z$ for $n \xi=z$. Supposing that $f^{\prime}(x+n \xi)=\varphi^{\prime}(z)$ is not infinite and has "the same sign" as $f^{\prime}(x+k \xi)$ $(k=0,1, \ldots, n-1)$, and that $z$ varies on any open interval like $(0, t)$ or $(-t, 0)$, the consequent of the implication (95)

\footnotetext{
${ }^{111}$ Lagrange specifies that this means these derivatives are all "positives" or all "negatives".

${ }^{112}$ Note that if the function $V(x, \xi)$ is identified with the ratio $\frac{f(x+\xi)-f(x)-\xi f^{\prime}(x)}{\xi}$, the statement that for any positive $\varepsilon$ the implication (89) holds for a certain positive $\delta$ reduces to the statement that the function $f(x)$ is differentiable. It follows that if one maintains that Lagrange identifies the function $V(x, \xi)$ with the ratio $\frac{f(x+\xi)-f(x)-\xi f^{\prime}(x)}{\xi}$, one also has to maintain that his argument is actually subject to the condition that any function is uniformly differentiable in an appropriate interval (Ovaert 1976, p. 190).
} 
reduces to

$$
\begin{array}{lllll}
0<\varphi(z)<2 z \Phi & \text { if } & z>0 & \text { and } & \varphi^{\prime}(z)>0 \\
-2 z \Phi<\varphi(z)<0 & \text { if } & z>0 & \text { and } & \varphi^{\prime}(z)<0 \\
2 z \Phi<\varphi(z)<0 & \text { if } & z<0 & \text { and } & \varphi^{\prime}(z)>0 \\
0<\varphi(z)<-2 z \Phi & \text { if } & z<0 & \text { and } & \varphi^{\prime}(z)<0
\end{array}
$$

where $z$ is supposed to vary on any interval $I_{z}$ like $(0, t]$ or $[-t, 0)$, the function $\varphi^{\prime}(z)$ is supposed not to become infinite in the corresponding open intervals like $(0, t)$ or $(-t, 0)$, and $\Phi=\max _{z \in I_{z}, z=0}\left|\varphi^{\prime}(z)\right|\left(\right.$ since $\max _{z \in I_{z}}\left|\varphi^{\prime}(z)\right| \geq \max _{k=1, \ldots, n}\left|f^{\prime}(x+k \xi)\right| \geq$ $\max _{k=1, \ldots, n-1}\left|f^{\prime}(x+k \xi)\right|$, and $\left.\left|f^{\prime}(x)\right|=\left|\varphi^{\prime}(0)\right|\right)$.

In fact, Lagrange explicitly writes neither the inequalities included in condition (96), nor any of the inequalities entering into our reformulation of his proof. He merely expresses these inequalities discursively. Instead of explicitly writing the inequalities included in the implication (95), he claims, for example, that "the quantity $f(x+n \xi)-f(x)$ will be [...] included between zero and $2 n \xi P$ ", where $P$ corresponds, in our notation, to $\pm F$. He therefore does not provide the exact meaning of the expression 'same sign', explicitly at any rate.

It is clear that Lagrange's argument does not require all the derivatives $f^{\prime}(x+k \xi)(k=0,1, \ldots, n-1)$ not to vanish, but only that their sum not vanish. It follows that, according to this argument, the inequalities included in conditions (96) hold even if the derivative $\varphi^{\prime}(z)$ is non-negative or non-positive in the interval $I_{z}$, provided it vanishes nowhere on the interval. Moreover, if all the derivatives $f^{\prime}(x+k \xi)(k=0,1, \ldots, n-1)$ vanished, for any choice of $\xi$ such that $0<|\xi| \leq \delta$, the function $f(x)$ would reduce to a constant. But in this case all the differences $f(x+(k+1) \xi)-f(x+k \xi)$ $(k=0,1, \ldots, n-1)$ would then vanish, and the function $\varphi(z)=f(x+n \xi)-f(x)$ would vanish too for any $z$ on $[0, t]$ or $[-t, 0]$. Hence if $\varphi^{\prime}(z)$ vanished everywhere on $[0, t)$ or $(-t, 0]$, then $\varphi(z)$ would vanish on $[0, t]$ or $[-t, 0]$.

As $\varphi(0)=0$ by hypothesis, this suggests the following reformulation of Lagrange's lemma:

Lemma 5. Suppose that $\varphi(z)$ is any function of $z$ such that $\varphi(0)=0$ and $t$ is a positive value of $z$ : if $0 \leq \varphi^{\prime}(z)<\infty$ on the interval $[0, t)$, then $0 \leq \varphi(z)<\infty$ on $[0, t]$; if $-\infty<\varphi^{\prime}(z) \leq 0$ on the interval $[0, t)$, then $-\infty<\varphi(z) \leq 0$ on $[0, t]$; if $0 \leq \varphi^{\prime}(z)<\infty$ on the interval $(-t, 0]$, then $-\infty<\varphi(z) \leq 0$ on $[-t, 0]$; if $-\infty<\varphi^{\prime}(z) \leq 0$ on the interval $(-t, 0]$, then $0 \leq \varphi(z)<\infty$ on $[-t, 0]$.

5.2.3. From the lemmas to the remainder theorem. Though Lagrange's proofs of his lemmas are actually flawed, he thought otherwise. He was probably also convinced that they hold for the limit-points of the intervals in question. Let us now see how they work in his proofs of the remainder theorem.

\subsubsection{THE PROOF OF THE Théorie.}

Let us begin with the proof offered in the Théorie (Lagrange 1797, arts. 45-53; 1813, arts. I.33-I.40; a reconstruction of Lagrange's proof is offered in Alvarez-Jimenez 1997, pp. 121-125).

From the power-series expansion theorem and theorem 4, according to the successive substitutions $x \rightarrow x-\xi$ and $\xi \rightarrow x z$, where $z$ is "any arbitrary quantity" (of course a variable one), Lagrange first obtains (Lagrange 1797, art. 45; 1813, art, I.33): ${ }^{113}$

$$
f(x)=\sum_{k=0}^{\infty} \frac{x^{k} z^{k}}{k !} f^{(k)}(x-x z) .
$$

Then (Lagrange 1797, art. 47; 1813, art. I.35), ${ }^{114}$ by applying to this expansion the same argument that leads to the equalities (79), he concludes that:

$$
f(x)=\sum_{k=0}^{h}\left[\frac{x^{k} z^{k}}{k !} f^{(k)}(x-x z)\right]+x^{h+1} R_{h+1}(x, z) \quad(h=0,1, \ldots),
$$

where $R_{h+1}(x, z)=z^{h+1} P_{h+1}(x-x z, x z)$ is a function of $x$ and $z$ that vanishes for $z=0$ (and that Lagrange seems to identify with the series $\left.\sum_{k=h+1}^{h} \frac{x^{k-h-1} z^{k}}{k !} f^{(k)}(x-x z)\right)$.

As this equality follows from the power-series expansion theorem and the theorem 4 for appropriate substitutions, the derivatives involved can be taken to be derivatives with respect to $x-x z$. To understand Lagrange's argument it is convenient to make it explicit (by using the same notational trick used in sections 3.3 and 4).

By deriving both members of the equalities (98) with respect to $z$, and observing that

$$
\left[f^{(k)_{x-x z}}(x-x z)\right]^{\prime z}=-x\left[f^{(k+1)_{x-x z}}(x-x z)\right]
$$

\footnotetext{
${ }^{113}$ From the equality (97), Lagrange derives en passant the Maclaurin expansion of any function, simply by supposing that $z=1$.

${ }^{114}$ We use the symbols ' $R_{h+1}(x, \xi)$ ' $(h=0,1, \ldots)$ to replace Lagrange's symbols ' $P$ ', ' $Q$ ', ' $R$ ', \&c.
} 
$(k=0,1, \ldots)$, Lagrange then obtains

$$
0=-x f^{\prime x-x z}(x-x z)+\sum_{k=1}^{h}\left[\begin{array}{r}
\frac{x^{k} z^{k-1}}{(k-1) !} f^{(k)_{x-x z}}(x-x z) \\
-\frac{x^{k+1} z^{k}}{k !} f^{(k+1)_{x-x z}}(x-x z) \\
+x^{h+1}\left[R_{h+1}(x, z)\right]^{\prime z}
\end{array}\right.
$$

and by simplification: ${ }^{115}$

$$
\left[R_{h+1}(x, z)\right]^{\prime z}=\frac{z^{h}}{h !} f^{(h+1)_{x-x z}(x-x z)} \quad(h=0,1, \ldots) .
$$

Since $R_{h+1}(x, 0)=0$, it follows that the remainders $R_{h+1}(x, z)$ are the primitives of $f^{(h+1)_{x-x z}(x-x z)} \frac{z^{h}}{h !}$ with respect to $z$ that vanish when $z$ does. ${ }^{116}$

To prove the remainder theorem, Lagrange has then to estimate these primitives. This is what the lemma of the Théorie is for (Lagrange 1797, arts. 49-53; 1813, art, I.39-40).

Suppose that $\varphi(z)$ is any function of $z$ whose first derivative has the form $z^{h} \phi(z)$, where $h$ is a non-negative integer and $\phi(z)$ is any other function of $z$. Suppose also that $a$ and $b(a<b)$ are two values of $z$ such that $\phi(z)$ is defined in $[a, b]$ and $M=\max _{z \in[a, b]} \phi(z)$ and $N=\min _{z \in[a, b]} \phi(z) .{ }^{117}$ Since, for $z \in[a, b]$, both the functions $z^{h}[M-\phi(z)]$ and $z^{h}[\phi(z)-N]$ are non-negative, from lemma 3 it follows that, if the interval $[a, b]$ does not contain an infinity of values of $z$ for which $z^{h}[M-\phi(z)]=z^{h}[\phi(z)-N]=0$, the differences between the primitives of these functions evaluated for $z=b$ and $z=a$ are non-negative as well: ${ }^{118}$

$$
\begin{aligned}
& \frac{M}{h+1}\left[b^{h+1}-a^{h+1}\right]+\varphi(a)-\varphi(b) \geq 0 \\
& \frac{N}{h+1}\left[a^{h+1}-b^{h+1}\right]+\varphi(b)-\varphi(a) \geq 0
\end{aligned}
$$

that is,

$$
\varphi(b) \leq \frac{M}{h+1}\left[b^{h+1}-a^{h+1}\right]+\varphi(a) \quad ; \quad \varphi(b) \geq \varphi(a)+\frac{N}{h+1}\left[b^{h+1}-a^{h+1}\right] .
$$

Let us now suppose that $\varphi(z)=R_{h+1}(x, z)$. From (101) it follows that:

$$
\varphi^{\prime}(z)=\frac{z^{h}}{h !} f_{x-x z}^{(h+1)}(x-x z) \quad \text { and } \quad \phi(z)=\frac{1}{h !} f_{x-x z}^{(h+1)}(x-x z) .
$$

As $R_{h+1}(x, 0)=\varphi(0)=0$, it suffices then to let $a=0$ and $b=1$ to infer, from the inequalities (103):

$$
z \in[0,1] \Rightarrow \frac{N_{h}}{(h+1) !} \leq R_{h+1}(x, 1) \leq \frac{M_{h}}{(h+1) !},
$$

where

$$
\begin{aligned}
& N_{h}=\min _{z \in[0,1]} f_{x-x z}^{(h+1)}(x-x z)=\min _{t \in[0, x]} f^{(h+1)}(t) \\
& M_{h}=\max _{z \in[0,1]} f_{x-x z}^{(h+1)}(x-x z)=\max _{t \in[0, x]} f^{(h+1)}(t) .
\end{aligned}
$$

${ }^{115}$ Lagrange details his argument leading from the equalities (98) to the equalities (101) only for $h=0$.

${ }^{116}$ By assuming that $x-x z=a$ this allows rewriting the equality (98) as

$$
f(x)=\sum_{k=0}^{h} \frac{(x-a)^{k}}{k !}\left[f^{(k)}(a)\right]+\frac{1}{h !} \int_{a}^{x}(x-t)^{h}\left[f^{(h+1)}(t)\right] d t .
$$

Lagrange leaves this implicit, however, probably to avoid the use of a definite integral.

${ }^{117}$ Once again, Lagrange's language is rather vague concerning the bounds of the interval he is considering:

Let $M$ be the greatest and $N$ the smaller value of $Z$ [i.e. $\phi(z)$, in our notation] for any value of $z$ included between the quantities $a$ et $b$.

Soit $M$ la plus grande, et $N$ la plus petite valeur de $Z$ pour toutes les valeurs de $z$ comprises entre les quantities $a$ et $b$.

(Lagrange 1797, and art. 49; 1813, art. I.39)

Still, the nature of his argument makes now it clear that the interval is closed. As remarked by Grabiner (1990, p. 155), Lagrange is here supposing that a function has a maximum and a minimum in a closed interval where it is defined, which, in modern terms, amounts to the theorem of intermediate values. But this is not a problem for him, since a later step in the proof (see p. 53, below) relies quite explicitly on the intermediate values property.

${ }^{118}$ Indeed Lagrange takes $z^{h}[M-\phi(z)]$ and $z^{h}[\phi(z)-N]$ to be "positif' and uses ' $>$ ' where we use ' $\geq$ '. But it is clear, from the definition of $M$ and $N$, that $M-\phi(z)=0$ and $[\phi(z)-N]=0$ for certain values of $z$ in the relevant interval. Thus we cannot see how to avoid taking Lagrange's adjective 'positif' as meaning 'non-negative' here, and his symbol ' $>$ ' as denoting the relation $\geq$. 
At this point Lagrange takes it for granted that if $R_{h+1}(x, 1)$ lies between two values taken by a function $f^{(h+1)}(t)$ in $[0, x]$ —as ensured by the implication (105) — the equality

$$
R_{h+1}(x, 1)=\frac{f^{(h+1)}(u)}{(h+1) !}
$$

holds for an appropriate $u$ in $[0, x]$. This is just the theorem of intermediate values, which he thus takes for granted.

If so, it suffices to assume that $z=1$ in the equality (98) to obtain:

$$
f(x)=\sum_{k=0}^{h}\left[\frac{x^{k}}{k !} f^{(k)}(0)\right]+\frac{x^{h+1}}{(h+1) !} f^{(h+1)}(u) \quad(h=0,1, \ldots),
$$

for an appropriate $u$ in $[0, x]$. The equality (62), and thus the remainder theorem, are finally obtained by generalization, that is, by interpreting the $x$ in $f(x)$ as $0+x$, then replacing 0 with $x$ and $x$ with $\xi$.

Once lemma 3 is accepted, much of Lagrange's proof reduces to a formal procedure. To carry out the proof, Lagrange nonetheless has to assume that the variable $z$ occurring in the general equality (98) takes the particular value $z=1$, and thus that the function $R_{h+1}(x, z)$ is defined for this particular value of $z$. This reduces the generality of such an equality. In moderns terms, this is the same as transforming the Taylor expansion of the function $f(x)$ centered on the indeterminate difference $x-x z$ into the Maclaurin expansion of the same function. Strictly speaking, the functions for which this last expansion is not defined should thus be excluded from the remainder theorem, given the way it is proved in the Théorie.

\subsubsection{THE PROOF FROM THE Leçons.}

It could be that this was one of the reasons for which Lagrange replaced, in the Leçons, the foregoing proof with a different one. The new proof goes as follows (Lagrange 1801, lecture IX; 1806, lecture IX).

For any function $f(x+\xi)$ in which the value of $x$ is supposed to be fixed, let $\rho_{h+1}$ and $\sigma_{h+1}(h=0,1,2, \ldots)$ be the values of $x+\xi$ such that

$$
f^{(h+1)}\left(\rho_{h+1}\right)=\max _{\xi \in[0, \eta]} f^{(h+1)}(x+\xi) \quad \text { and } \quad f^{(h+1)}\left(\sigma_{h+1}\right)=\min _{\xi \in[0, \eta]} f^{(h+1)}(x+\xi),
$$

where $\eta$ is any given positive quantity. ${ }^{119}$ It follows that when $\xi$ belongs to $[0, \eta]$, the differences $f^{(h+1)}\left(\rho_{h+1}\right)-$ $f^{(h+1)}(x+\xi)$ and $f^{(h+1)}(x+\xi)-f^{(h+1)}\left(\sigma_{h+1}\right)$ are both non-negative. ${ }^{120}$ Hence, according to lemma 5 , if $f^{(h+1)}(x+$ $\xi$ ) becomes infinite for no value of $\xi$ in $[0, \eta]$ (so that $f^{(h+1)}\left(\rho_{h+1}\right)$ and $f^{(h+1)}\left(\sigma_{h+1}\right)$ are finite, and the differences $f^{(h+1)}\left(\rho_{h+1}\right)-f^{(h+1)}(x+\xi)$ and $f^{(h+1)}(x+\xi)-f^{(h+1)}\left(\sigma_{h+1}\right)$ become infinite for no value of $\xi$ in $\left.[0, \eta]\right)$, the primitives of these differences (with respect to $\xi$ ) are both non-negative when $\xi$ belongs to [0, $\eta$ ].

By evaluating the constants that enter into these primitives for these same primitives to be null for $\xi=0$, one obtains

$$
\begin{gathered}
\xi f^{(h+1)}\left(\rho_{h+1}\right)+f^{(h)}(x)-f^{(h)}(x+\xi) \geq 0 \\
\quad \text { and } \\
f^{(h)}(x+\xi)-\xi f^{(h+1)}\left(\sigma_{h}\right)-f^{(h)}(x) \geq 0 .
\end{gathered}
$$

Insofar as $f^{(h)}(x+\xi)$ becomes infinite for no value of $\xi$ in $[0, \eta]$, a further application of lemma 5 leads to

$$
\begin{aligned}
& \frac{\xi^{2}}{2} f^{(h+1)}\left(\rho_{h}\right)+\xi f^{(h)}(x)+f^{(h-1)}(x)-f^{(h-1)}(x+\xi) \geq 0 \\
& \text { and } \\
& f^{(h-1)}(x+\xi)-\frac{\xi^{2}}{2} f^{(h+1)}\left(\sigma_{h}\right)-\xi f^{(h)}(x)-f^{(h-1)}(x) \geq 0 .
\end{aligned}
$$

It is thus enough to apply lemma $5 h+1$ times to obtain

$$
\frac{\xi^{h+1}}{(h+1) !} f^{(h+1)}\left(\sigma_{h+1}\right)+\sum_{k=0}^{h} \frac{f^{(k)}(x)}{k !} \xi^{k} \leq f(x+\xi) \leq \frac{\xi^{h+1}}{(h+1) !} f^{(h+1)}\left(\rho_{h+1}\right)+\sum_{k=0}^{h} \frac{f^{(k)}(x)}{k !} \xi^{k},
$$

${ }^{119}$ Lagrange's language is again vague on the bounds of the interval in question. Concerning the case $h=0$ he writes: Let firstly $p$ and $q$ [i.e. $\sigma_{1}$ and $\rho_{1}$ in our notation] the values of $x+\xi$ that make the derivative function $f^{\prime}(x+\xi)$ is the smallest and the greatest, en regrading $x$ as given, and and letting $\xi$ vary from zero up to whatever given value of $\xi$.

Soient d'abord $p$ et $q$ [that is, $\sigma_{1}$ and $\rho_{1}$ in our notation] les valeurs de $x+\xi$ qui rendent la function dérivée $f^{\prime}(x+\xi)$ la plus petite et la plus grande, en regardand $x$ comme donné, et faisant varier $\xi$ depuis zéro jusqu'à une valeur quelconque donnée de $\xi$.

(Lagrange 1801, p. 70; 1806, pp. 93-94.)

Here again the argument seems to make it clear that the interval is closed. As $x$ is supposed to be fixed, but indeterminate, the existence of a value like $\eta$ such that the function $f^{(h)}(x+\xi)$ is defined when $\xi$ belongs to $[0, \eta]$ is not a problem for Lagrange. It follows that he is here supposing, as in his proof of the Théorie (see footnote 117 above), that a function has a maximum and a minimum in a closed interval where it is defined. But even here this poses no problem, since a subsequent step of his proof relies quite explicitly on the assumption of the intermediate values property.

${ }^{120}$ Lagrange also takes the differences $f^{(h+1)}\left(\rho_{h+1}\right)-f^{(h+1)}(x+\xi)$ and $f^{(h+1)}(x+\xi)-f^{(h+1)}\left(\sigma_{h+1}\right)$ to be "positives" and writes ' $>$ ' where we write ' $\geq$ '. Still, from the definition of $\rho_{h+1}$ and $\sigma_{h+1}$ it follows that these differences vanish for certain values of $\xi$ in $[0, \eta]$. Here again it seems one has to take 'positif' to mean 'non-negative' and ' $>$ ' to denote the relation $\geq$. 
whenever the derivatives $f^{(h+1)}(x+\xi)$ do not become infinite for $\xi$ in $[0, \eta]$. An similar argument works for $\xi$ in $[-\eta, 0]$, and leads to a similar result:

$$
\frac{\xi^{h+1}}{(h+1) !} f^{(h+1)}\left(\rho_{h+1}\right)+\sum_{k=0}^{h} \frac{f^{(k)}(x)}{k !} \xi^{k} \leq f(x+\xi) \leq \frac{\xi^{h+1}}{(h+1) !} f^{(h+1)}\left(\sigma_{h+1}\right)+\sum_{k=0}^{h} \frac{f^{(k)}(x)}{k !} \xi^{k},
$$

where $\rho_{h+1}$ and $\sigma_{h+1}$ are the values of $x+\xi$ such that

$$
f^{(h+1)}\left(\rho_{h+1}\right)=\max _{\xi \in[-\eta, 0]} f^{(h+1)}(x+\xi) \quad \text { and } \quad f^{(h+1)}\left(\sigma_{1}\right)=\min _{\xi \in[-\eta, 0]} f^{(h+1)}(x+\xi),
$$

whenever the derivatives $f^{(h+1)}(x+\xi)$ do not become infinite when $\xi$ belongs to $[-\eta, 0]$.

If the theorem of intermediate values is taken for granted, the inequalities (111) and (112) taken together are equivalent to the remainder theorem. However, instead of formulating the theorem explicitly in full generality, Lagrange merely points out that the inequalities (111) and (112) provide the limits of the remainder of the power-series expansion of any function $f(x+\xi)$ prolonged up to any finite order, gives some examples (Lagrange 1801, pp. 74-77; 1806, pp. 99-103), and deduces the equality (107), which is now understood as a particular consequence of a more general result (Lagrange 1801, p. 78; 1806, p. 105).

By discussing Lagrange's proof of the lemma of the Leçons, we have observed that he tries-unsuccessfully-to free this proof from the assumption that any function $f(x+\xi)$ has, for any $x$ for which $f(x)$ is defined and is not infinite, a generalized power-series expansion that converges to such a function around $\xi=0$. This is confirmed by the previous argument. To obtain the inequalities (111) and (112), Lagrange constructs step by step the partial sum $\sum_{k=0}^{h} \frac{f^{(k)}(x)}{k !} \xi^{k}$ of any order $h$, starting from the derivative $f^{(h+1)}(x+\xi)$, considering its maximum and minimum in an appropriate interval centered on $\xi=0$ and applying the algorithm of the primitives. Supposing that this algorithm and the derivatives entering into his construction are given independently of the consideration of any expansion of $f(x+\xi)$, this procedure is completely independent of any presupposition of convergence and suggests the modern proof of the remainder theorem, which is based on the definition of the remainder as an appropriate finite difference.

It is Lagrange himself that suggests the possibility of understanding the remainder in such a way, when he applies the inequalities (111) and (112) to the example of the function $x^{m}$ :

Through these limits [those of the reminder of the power-series expansion of $(x+\xi)^{m}$ ], one is preserved from the difficulties that can result from the non-convergence of the series, since, as whatever $n$-th term is to the following in the ratio of 1 to $\frac{m-n+1}{n} \frac{\xi}{x}$, in order for the series to be convergent, it is necessary that the quantity $\frac{m-n+1}{n} \frac{\xi}{x}$ be, independently of the sign it has to have, smaller than the unity. If $\frac{\xi}{x}<1$, it is clear that the series will always become convergent, since the last value of $\frac{m-n+1}{n}$ is -1 .

Par le moyen de ces limites, on est à couvert des difficultés qui peuvent résulter de la non-convergence de la série; car, comme un terme quelconque $n^{\text {ème }}$ est au suivant dans le rapport de 1 à $\frac{m-n+1}{n} \frac{\xi}{x}$, pour que la série soit convergente, il faut que la quantité $\frac{m-n+1}{n} \frac{\xi}{x}$, abstraction faite du signe qu'elle doit avoir, soit moindre que l'unité. $\mathrm{Si} \frac{\xi}{x}<1$, il est clair que la série finira toujours par être convergente, puisque la dernière valeur de $\frac{m-n+1}{n}$ est -1 .

(Lagrange 1801, p. 75; 1806, p. 100.)

Though Lagrange does not use the remainder theorem to evaluate the convergence of his series (he uses d'Alembert's test instead: Alembert 1768, p. 171), it is clear that he supposes here that the equality

$$
(x+\xi)^{m}=\sum_{k=0}^{h}\left(\begin{array}{c}
m \\
k
\end{array}\right) x^{m-k} \xi^{k}+P_{h+1}(x, \xi) \xi^{h+1}
$$

(where $P_{h+1}(x, \xi)=\left(\begin{array}{c}m \\ h\end{array}\right)(x+j)^{x-h-1}$, for an appropriate $j$ in $\left.[0, \xi]\right)$ holds even when the power-series expansion of this $(x+\xi)^{m}$ is divergent.

But if this expansion is not taken to converge to the function $(x+\xi)^{m}$ on an appropriate interval, how can Lagrange define the derivative of $x^{m}$ ? And, more generally, how can he assume the algorithms of the primitives and the derivatives to be given without assuming that there is a power series converging to any function $f(x+\xi)$ around $\xi=0$ ?

No consistent answer seems to be possible. Lagrange was possibly thinking that the step-by-step procedure included in the proof of the remainder theorem offered in the Leçons manages to obtain, as it were, constructively, the partial sum $\sum_{k=0}^{h} \frac{f^{(k)}(x)}{k !} \xi$. This is what the following passage, occurring at the end of this proof, suggests:

The foregoing analysis successively provides again, as one can see, the terms of the expansion of $f(x+$ $\xi)$. But it is has the advantage of expounding this function as far as one wishes, and of providing the limits of the remainder. 
L'analyse précédente redonne, comme l'on voit, successivement les termes du développement de $f(x+\xi)$; mais elle a l'avantage de ne développer cette fonction qu'autant que l'on veut, et d'offrir des limites du reste.

(Lagrange 1801, p. 73; Lagrange 1806, p. 98.)

However, this advantage can at most be operational, since Lagrange's theory collapses if the existence of a powerseries expansion of any function $f(x+\xi)$ is not previously assumed. Though many of the shortcomings of Lagrange's theory are reflected in this difficulty, his attempt to free his proof of the remainder theorem from a previous supposition of convergence also shows his capacity to see beyond the bounds of this theory and suggests a possible alternative.

This explains why the remainder theorem has been one of the few original results contained in the Théorie and the Leçons that have survived the theory, and why this has been possible only thanks to a dramatic change in the role of this theorem within the system of the calculus.

\section{Conclusions}

In a sense, both the Théorie and the Leçons are more philosophical essays than treatises intended to obtain particular mathematical results. They are part of a foundationalist agenda. The fact that this agenda was never really accepted by Lagrange's contemporaries contrasts with another fact: that it was the most careful attempt to integrate the calculus within the program of 18th-century algebraic analysis. Its failure is then also the failure of this ambitious program.

We hope our account not only contribute to a better understanding of Enlightenment mathematics, but also shows, on a relevant example, that the problem of the foundation of mathematics is by no means peculiar to twenty-century mathematics. It has been addressed before in different occasions and in different ways. For Lagrange, it was not a problem of the certainty of the ultimate bases of mathematics. It was a matter of generality and internal organization; it was essentially a question of purity of method.

\section{ANNEX}

The first edition of the Théorie (Lagrange 1797) appeared on Prairial and V (May 20th to June 18th 1797) at the Imprimerie de la République, while the Leçons were firstly published in 1801 (Lagrange 1801), as the tenth volume of the second edition of the Leçons de École Normale de l'an III. This was an addition to the lectures actually delivered at the École Normale de l'an III, since Lagrange's five lectures at this school were devoted to more elementary subjects. ${ }^{121}$.

Many documents attest that Lagrange taught his theory of analytical functions at the École Polytechnique between 1795 and 1799. When the École centrale des travaux publics — which later became the École polytechnique—was created in 1794, Lagrange was made president of the Conseil de l'École, but he only taught there from Spring 1795 (Dahan 1992,179-180).

A note contained in the third volume of the Correspondance sur l'École Polytechnique (1814, 1, p. 93) attests that the Théorie and the Leçons include the matter taught during the years 1795, 1796 and 1799.

In introducing his course for the an VII (on 7th Pluviôse an VII: January 26th, 1799) Lagrange presented it as being devoted to "the theory of functions", claiming that his aim was to expound this theory "with more detail than in the printed work" (Lagrange 1799, p. 232). This suggests that the Théorie includes the matter taught in 1795 and 1796 , and the Leçons the matter taught in 1799.

This is confirmed by a note included in certain copies of the first edition of the Théorie, bound as the ninth cahier of the Journal de l'École Polytechnique (see for example the copy digitalized by Gallica: FRBNF36048706), which first appeared an IX (September 23th, 1800 to September 22th, 1801), where it is explicitly said that "the Théorie des fonctions analytiques [...] has been the subject of the lectures delivered by Mr. Lagrange at this École in 1795 and 1796", while "in $1799 \mathrm{Mr}$. Lagrange has taken up the calcul de fonctions as subject of his lectures at the École polytechnique" in delivering twenty lectures published in the twelfth cahier of the same Journal.

This refers to a reprint of the first edition of the Leçons (including some minor revisions), which first appeared in Thermidor an XII (July 20th to August 18th, 1804), where the Leçons are presented as a "commentary and a supplement" to the Théorie, corresponding to Lagrange's course at the École for 1799 (for further details on the publication of the Leçons, see Grattan-Guinness 1990, I, 195-196).

Again, a note by de Prony in the second cahier of the Journal de l'École Polytechnique, which first appeared on Nivôse and IV (December 22th, 1995 to January 20th, 1996), mentions "an elementary course of analysis" that Lagrange gave at

${ }^{121}$ See: Ecole Normale III (D), vol. I, pp. 34-55, lecture of 16th Pluviôse (February 4th, 1795); Ecole Normale III (L) vol. III, pp. 227-253, 276-310, 463-489, lectures of 6th Ventôse (February 24th, 1795), 1st Germinal (March 21th, 1795), and 6th Germinal (March 26th, 1795), respectively; and vol. IV, pp. 401-420, lecture of 22th Germinal (April 11th, 1795). The same lectures are also reprinted with the same pagination in Ecole Normale III (NE), vol. I, Débats (lecture of 16th Pluviôse), vol. III, Leçons (lectures of 6th Ventôse, and 1st and 6th Germinal); vol. IV, Leçons (lecture of 22th Germinal). Lagrange also participated in a debate, with Laplace, on 11th Pluviôse (January 30th, 1795), and was present when Laplace read the program of all the mathematical lectures on first Pluviôse (January 20th, 1795): Ecole Normale III (D), vol. I (and NE, vol. I, Débats), pp. 5-23; (L), vol. I (and NE, vol. I, Leçons), pp. 16-21. Lagrange's lectures and his and Laplace's interventions in the debate of the 11th Pluviôse have been more recently published in Dhombres (1992), pp. 193-265, by A. Daham-Dalmédico. The program read by Laplace on the first Pluviôse is also published there, pp. 45-47. 
the École from the 5th Prairial and IV (May 24th, 1796), where he presented "a matter belonging only to himself whose object is the proof of the fundamental principles of the differential and integral calculus" (Prony 1796. p. 208).

That Lagrange actually presented his theory at the École Polytechnique in 1795-1796 is, however, called into question by the manuscript 1323 of the library of the École des Ponts et Chausses containing an account of lectures given by Lagrange at the École Polytechnique in 1796 and 1797: only one of these lectures has to do with the theory of analytical functions; this was delivered between the 5th and the 16th Messidor an VIII (June 23th to July 4th, 1797), and is nothing but a general presentation of the Théorie, whose first edition had appeared about a month before (this lecture has been edited in Pepe 1986).

After 1799, Lagrange only returned to his treatises to make additions and changes in style and organization. The most relevant additions concern the calculus of variations.

The second edition of the Leçons (Lagrange 1806), which came out in 1806, includes, among many more local changes, two new lectures on this matter, which had received no attention in the first edition (on Lagrange's treatment of the calculus of variations in these lectures, see Fraser 1987, pp. 49-50; on his different approaches to this subject at different stages of his career see Fraser 1985).

The second edition of the Théorie (Lagrange 1813), which appeared in 1813 - the year of Lagrange's death—only contains local changes, most of which are purely stylistic.

The following four tables show the organization of the various subjects dealt with in Lagrange's treatises. The first shows how the four components mentioned on page 4 are distributed. The three others show how the different topics that components (ii) and (iv) concern enter into these treatises.

In all the tables, the Arabic and Roman numerals refer to the articles of the Théorie and the lectures of the Leçons, respectively. 
TABLE 1. The Structure of Lagrange's Treatises

\begin{tabular}{|c|c|c|c|c|}
\hline \multicolumn{5}{|c|}{ THE STRUCTURE OF LAGRANGE's TREATISES } \\
\hline & Théorie, 1797 & Leçons, 1801 & Leçons, 1806 & Théorie, 1813 \\
\hline \multirow{3}{*}{ (i) } & $1-10$ & I-VI & I-VI & $\begin{array}{c}\text { Introduction } \\
1-2, \text { part 1 } \\
\text { 8-32, part 1 }\end{array}$ \\
\hline \multirow{2}{*}{ (ii) } & $16-44$ & VIII & VIII & 41-95, part 1 \\
& $54-107$ & VII & VII & $61-76$, part 2 \\
\hline \multirow{2}{*}{ (iii) } & $170-184$ & X-XX & X-XXII & I-7, part 1 \\
& $11-15$ & IX & IX & $33-40$, part 1 \\
\hline \multirow{3}{*}{ (iv) } & $45-53$ & & & $1-60$, part 2 \\
& $108-169$ & & $\backslash$ & $77-87$, part 2 \\
& $185-228$ & & & $1-47$, part 3 \\
\hline
\end{tabular}

TABLE 2. Reformulation of the edifice of the Calculus in its pure part

\begin{tabular}{|c|c|c|c|c|}
\hline \multicolumn{5}{|c|}{ REFORMULATION OF THE EDIFICE OF THE CALCULUS IN ITS PURE PART } \\
\hline & Théorie, 1797 & Leçons, 1801 & Leçons, 1806 & Théorie, 1813 \\
\hline $\begin{array}{l}\text { Derivative of a function } \\
\text { with respect to any } \\
\text { function of its variable }\end{array}$ & I & VII & VII & / \\
\hline $\begin{array}{l}\text { Derivative equations } \\
\text { and transformation } \\
\text { of functions }\end{array}$ & $54-57$ & $\mathrm{X}$ & $\mathrm{X}$ & $41-44$ \\
\hline $\begin{array}{l}\text { Formulas relative to } \\
\text { to trigonometric } \\
\text { functions }\end{array}$ & / & $\mathrm{X}-\mathrm{XI}$ & $\mathrm{X}-\mathrm{XI}$ & / \\
\hline $\begin{array}{l}\text { Arbitrary constants } \\
\text { occurring in the } \\
\text { solution of derivative } \\
\text { equations }\end{array}$ & $58-61$ & XII & XII & $45-48$ \\
\hline $\begin{array}{l}\text { Solution of some } \\
\text { derivative equations }\end{array}$ & $\begin{array}{l}62-70 \\
79-84\end{array}$ & / & / & $\begin{array}{l}49-57 \\
67-72\end{array}$ \\
\hline $\begin{array}{l}\text { Theory of singular } \\
\text { primitives }\end{array}$ & $71-76$ & XV-XVIII & XIV-XVII & $58-63$ \\
\hline $\begin{array}{l}\text { Applications to series and } \\
\text { equations of } 3^{r d} \text { degree }\end{array}$ & $76-78$ & / & / & $64-66$ \\
\hline $\begin{array}{l}\text { Expansion of any function } \\
\text { of a root of the equation } \\
z=x+f(z)\end{array}$ & $97-99$ & / & / & $85-87$ \\
\hline $\begin{array}{l}\text { Function of several } \\
\text { variables and partial } \\
\text { derivative equations }\end{array}$ & $\begin{array}{c}85-96 \\
100-107\end{array}$ & XIV and XX & XIX-XXI & $\begin{array}{l}73-84 \\
88-95\end{array}$ \\
\hline Theory of the Multiplier & I & XIII & XIII & / \\
\hline $\begin{array}{l}\text { Finite difference } \\
\text { equations }\end{array}$ & / & XIX & XVIII & l \\
\hline Calculus of variations & $170-184$ & I & XXI-XXII & $61-76$, part 2 \\
\hline
\end{tabular}


TABle 3. Geometrical Applications

\begin{tabular}{|l|c|c|}
\hline \multicolumn{3}{|c|}{ GEOMETRICAL APPLICATIONS } \\
\hline & Théorie, 1797 & Théorie, 1813 \\
\hline $\begin{array}{l}\text { Tangents and points } \\
\text { of contact of curves }\end{array}$ & $108-130$ & $1-19$, part 2 \\
\hline Maxima and minina & $\begin{array}{l}131-133 \\
160-169\end{array}$ & $\begin{array}{l}20-26, \text { part 2 } \\
51-60, \text { part 2 }\end{array}$ \\
\hline $\begin{array}{l}\text { Quadrature and } \\
\text { rectification }\end{array}$ & $134-136$ & $27-29$, part 2 \\
\hline $\begin{array}{l}\text { Curves in space } \\
\text { and surfaces }\end{array}$ & $139-159$ & $32-50$, part 2 \\
\hline $\begin{array}{l}\text { Volumes and } \\
\text { areas of surfaces }\end{array}$ & $137-138$ & $\begin{array}{l}30-31, \text { part 2 } \\
77-87, \text { part 2 }\end{array}$ \\
\hline
\end{tabular}

TABLE 4. Mechanical Applications

\begin{tabular}{|l|c|c|}
\hline \multicolumn{3}{|c|}{ MECHANICAL APPLICATIONS } \\
\hline $\begin{array}{l}\text { Speed, acceleration } \\
\text { and force }\end{array}$ & $185-189$ & $1-6$, part 3 \\
\hline $\begin{array}{l}\text { Composition of motions } \\
\text { and forces }\end{array}$ & $190-194$ & $7-10$, , part 3 \\
\hline $\begin{array}{l}\text { Curvilinear motion and } \\
\text { equation of motion }\end{array}$ & $195-200$ & $11-16$, part 3 \\
\hline $\begin{array}{l}\text { Motion in a } \\
\text { resistant medium }\end{array}$ & $201-205$ & $17-24$, part 3 \\
\hline $\begin{array}{l}\text { Motion on a surface and } \\
\text { principle of virtual velocity }\end{array}$ & $206-210$ & $25-30$, part 3 \\
\hline $\begin{array}{l}\text { Centre of gravity and } \\
\text { rotation of surfaces }\end{array}$ & $211-218$ & $31-38$, part 3 \\
\hline $\begin{array}{l}\text { Vis Viva } \text { and } \\
\text { conservation of energy }\end{array}$ & $219-228$ & $39-47$, part 3 \\
\hline
\end{tabular}

We are very much indebted to Jean Dhombres and Alexander Afriat who read previous drafts of this paper and offered many general and particular suggestions. We also thank Carlos Alvarez, Andrew Arana, Tom Archibald, Emmanuel Barot, June Barrow-Green, Karine Chemla, Annalisa Coliva, Benno van Dalen, Marie-José Durand-Richard, Craig Fraser, Massimo Galuzzi, Christian Gilain, Jeremy Gray, Niccolò Guicciardini, Jesper Lützen, Antoni Malet, Paolo Mancosu, Philippe Nabonnand, Eduardo Noble, Göran Sundholm, Jamie Tappenden, for valuable remarks and suggestions.

\section{REFERENCES}

F. U. T. Æpinus (1760-1761). Demostratio generalis theorematis newtoniani de binomio ad potentiam indefinitam elevando. Novi Commentarii academiae scientiarum imperialis Petropolitanae, 8:169-180 (abstract at pp. 27-29 of Summarium Dissertationum), 1760-1761. Publ. 1763. French Translation (by J. Dhombres and M. Pensivy) in: Sciences and Techniques en Perspective, 11, 1986-1987, pp. 204-218.

J. B. le R. d' Alembert (DIS). Discours préliminaire des éditeurs. In Encyclopédie, ou dictionnaire raisonné des sciences, des arts et des métiers, Briasson, David l'aîné, le Breton, Durand, Paris, 1751-1780, 35 volumes, vol. 1 (1751), pp. IXLV.

J. B. le R. d' Alembert (ANA). Analytique. In Encyclopédie, ou dictionnaire raisonné des sciences, des arts et des métiers, Briasson, David l'aîné, le Breton, Durand, Paris, 1751-1780, 35 volumes, vol. 1 (1751), pp. 403-404.

J. B. le R. d' Alembert (1768). Refléxion sur les suites et sur les racine imaginaires. In Opuscles mathématiques, David, Briasson, C. A. Jombert, Paris, 1761-1780 (8 tomes), tome 5 (1768), pp. 171-215.

C. Alvarez-Jimenez (1997). Mathematical analysis and analytical science. In M.Otte and M. Panza, ed., Analysis and Synthesis in Mathematics. History and Philosophy, (vol. 196 of Boston Studies in the Philosophy of Science), Kluwer A. P., 1997, pp. 103-145.

A. M. Ampère (1806). Recherches sur l'application des formules générales du calcul des variations aux problèmes de la mécanique Mémoires présentés à l'Institut des sciences, lettres et arts, par divers savans, et lus dans ses assemblées. 
Sciences mathématiques et Physiques, tome I, January 1806, pp. 493-523. Presented on 26th Floréal an XI (May 16th, 1803).

A. Arana (2008). Logical and semantic purity. Protosociology, 25, 2008, pp. 36-48.

L. F. A. Arbogast (ESSAI). Essai sur des nouveaus principes de calcul difféérentiel et de calcul intégral. Manuscript conserved at the Biblioteca Medicea-Laurenziana in Florence (Cod. Laur. Ashb. App. 1840) and at the Bibliothèque de l'École des Ponts et Chaussées in Paris (Ms. 2809).

Johann Bernoulli (1718). Remarques sur ce qu'on a donné jusqu'ici de solutions des problèmes sur les isoperimétres. Histoire de l'Académie Royale des Sciences [de Paris], Mémoires de Mathématiques et Physique, 1, 1718 (2 tomes published in 1719 and 1720), pp. 100-138 of tome I.

B. P. J. N. Bolzano (1817). Rein analytischer Beweis des Lehrsatzes [... ]. G. Haase, Prag, 1817 (Abhandlungen der Königl. Böhmischen Gesellschaft der Wissenschaften, 5: 1-60).

E. Borel (1929). Leçons sur les séries devergentes. Gauthier-Villars, Paris, 1929.

U. Bottazzini (1986). The Higher Calculus. A History of Real and Complex Analysis from Euler to Weierstrass. Springer, New York, 1986.

U. Bottazzini (1990). Geometrical rigour and 'modern analysis'. An introduction to Cauchy's Cours d'analyse. In Cauchy (CAB), pp. XI-CLVII.

L. Carnot (1797). Réfléxions sur la métaphysique du calcul infinitésimal. Duprat, Paris, an V (1797). 2nd edition, M. V. Courcier, Paris, 1813.

A. L. Cauchy (1821). Cours d'analyse de l'École royale polytechnique [...], Ire partie. Analyse algébrique. Debure frères, Paris, 1821. Also in Cauchy (EUVRES), ser. 2 vol. III.

A. L. Cauchy (1822). Sur le développement des fonctions en séries, et sur l'intégration des équations différéntielles, ou aux différences partielles. Bull. des Sci. par la Soc. Philomatique de Paris, 1822, pp. 49-54. Also in Cauchy (EUVRES), ser. 2 vol. II, pp. 276-282. References are relative to this last edition.

A. L. Cauchy (1823). Résumé des leçons données à l'École royale polytechnique sur le calcul infinitésimal. Impr. Royale, chez Debure, Paris, 1823. Also in Cauchy (CEUVRES), ser. 2 vol. IV.

A. L. Cauchy (EUVRES). CEuvres Complètes. Gauthier-Villars et fils, Paris, 1882-1903, 27 volumes in 2 series.

A. L. Cauchy (CAB). Cours d'analyse de l'école royale polytechique. Première partie. Analyse algèbrique. CLUEB, Bologna, 1990. Edited by U. Bottazzini.

A. L. Cauchy (CABS). Cauchy's Cours d'analyse. An Annotated Translation. Springer, Dordrecht, Heidelberg, London, New York, 2009. Translation and notes by R. E. Bradley and C. E. Sandifer.

E. B. Condillac (1797). La langue des calculs. Imp. de Ch. Houel, Paris, an VI (1797).

M. J. A. C. Condorcet (1765). Du calcul intégral. Didot, Paris, 1765.

A. Dahan Dalmédico (1992). La méthode critique du “mathématicien-philosophe”. In Dhombres (1992), pp. 171-192.

J.-T. Desanti (1973). Notes sur l'épistémologie égélienne; l'intériorisation du concept. Dialectique, 1-2, 1973 , pp. $55-87$. Re-published in J.-T. Desanti, La philosophie silencieuse ou critique de la philosophie de la science, Seuil, Paris, 1975, pp. 22-66.

R. Descartes (1637). Discours de la méthode [...]. Plus la Dioptrique. Les météores. Et la Géométrie qui sont des essaies de cette Méthode. I. Maire, Leyde, 1637. Re-edited in Descartes AT, vol. VI, pp. 367-485.

R. Descartes (AT). Euvres de Descartes, ed. by C. Adam et P. Tannery, Vrin, Paris, 1897-1910, 12 volumes.

M. Detlefsen (2008). Purity as an ideal of proof. In Mancosu (2008), pp. 179-197.

J. Dhombres (1982-1983). La langue des calculs de Condillac (ou comment propager les lumières?). Sciences et techniques en perspective, 2, 1982-1983, pp. 197-230.

J. Dhombres (1987). Sur un texte d'Euler relatif à une équation fonctionnelle. Archaïsme, pédagogie et style d'écriture. Historia Scientiarum, 33, 1987, pp. 77-123.

J. Dhombres (1988). Un texte d'Euler sur les fonctions continues et le fonctions discontinues, véritable programme d'organisation de l'analyse au 18e siècle. Cahiers du Séminaire d'Histoire des Mathématiques, 9, 1988, pp. 23-97. Including a French translation of Euler (1765).

J. Dhombres, ed. (1992). L'École Normale de l'an III. Leçons de mathématiques. Laplace - Lagrange - Monge. Paris, Dunod, 1992.

J. Dhombres and M. Pensivy (1988). Ésprit de rigueur et présentation mathématique au XVIIIème siècle : le cas d'une démonstration d' Epinus. Historia Mathematica, 15, 1988, pp. 9-31.

P. Dugac (2003). Histoire de l'analyse. Autour de la notion de limite et de ses voisinages. Paris, Vuibert, 2003.

M. Dummett (1991). Frege. Philosophy of Mathematics. London, Duckworth, 1991.

Ecole Normale III (L). Séances des écoles normales, recueillies par des sténographes et revues par les professeurs. Première partie. Leçons. L. Reynier, Paris, s.d., 6 volumes.

Ecole Normale III (D). Séances des écoles normales, recueillies par des sténographes et revues par les professeurs. Seconde partie. Débats. Impr. du Cercle Social, Paris, s.d., 3 volumes. 
Ecole Normale III (NE). Séances des écoles normales, recueillies par des sténographes et revues par les professeurs, nouvelle édition. Impr. du Cercle Social, Paris, 1800-1801, 13 volumes (1-10, Leçons + 1-3, Débats).

L. Euler (1744). Methodus inveniendi lineas curvas [‥]. M.-M. Bousquet \& Soc., Lausanne \& Genevæ, 1744. Also in Euler (OPERA), ser. 1, vol. XXIV.

L. Euler (1748). Introductio in analysisn infinitorum. M.-M. Bousquet \& Soc., Lausanne, 1748, 2 volumes. Also in Euler (OPERA), ser. 1, volumes VIII et IX.

L. Euler (1749). De vibratione chordarum exercitatio. Nova Acta Eruditorum, 1749, pp. 512-527. Also in Euler (OPERA), ser. 2, vol. 10, pp. 50-62.

L. Euler (1753). Remarques sur les mémoires précédens de M. Bernoulli. Histoire de l'Académie Royale des Sciences [de Paris], Mémoires de Mathématiques et Physique, 9, 1753, pp. 196-222. Also in Euler (OPERA), ser. 2, vol. 10, pp. 233-254.

L. Euler (1765). De usu functionum discontinarum in analysi. Novi Commentarii academiae scientiarum Imperialis Petropolitana, 11, 1765, pp. 3-27 (abstract at pp. 3-7 of Summarium Dissertationum). Also in Euler (OPERA), ser. 1, vol. 23, pp. 74-91.

L. Euler (1787). Nova demonstratio quod evolutio potestatum binomii neutoniana etiam pro exponentibus fracti valeat. Nova acta academiae scientiarum imperialis Petropolitana, 5, 1787, pp. 52-58, Also in Euler (OPERA), ser. 1, vol. 16/1, pp. 112-121.

L. Euler (OPERA). Leonhardi Euleri Opera omnia. Soc. Sci. Nat. Helveticæ, Leipzig, Berlin, Basel, 1911-... 76 volumes published to date.

L. Euler (BLA). Introduction to the Analysis of the Infinite. Springer-Verlag, New York, 1988-1990, translation by J. D. Blanton, 2 volumes.

G. Ferraro (2000). Functions, functional relations and the laws of continuity in Euler. Historia Mathematica, 27, 2000, pp. 107-132.

G. Ferraro (2007). The foundational aspects of Gauss's work on the hypergeometric, factorial and digamma functions. Archive for History of Exact Sciences, 61, 2007, pp. 457-518.

G. Ferraro and M. Panza (2003). Developing into series and returning from series. A note on the foundation of 18thcentury analysis. Historia Mathematica, 30, 2003, pp. 17-46, .

C. Fraser (1985). J. L. Lagrange's changing approach to the foundation of the calculus of variations. Archive for History of Exact Sciences, 39, 1985; pp. 151-191.

C. Fraser (1987). Joseph Louis Lagrange algebraic vision of the calculus. Historia Mathematica, 14, 1987, pp. 38-53.

C. Fraser (1989). The calculus as algebraic analysis: Some observations on mathematical analysis in the 18th century. Archive for History of Exact Sciences, 39, 1989, 317-335.

E. Galois (EMM). Écrits et mémoires mathématiques d'Evariste Galois, ed. by R. Bourgne et J.-P. Azra, 2nd edition, Gauthier-Villars, Paris, 1976.

C. F. Gauss (WERKE). Werke. Königlichen Gesellshaft der Wissenschaften, Göttingen, 1863-1933,12 volumes.

C. Gilain (1988). Condorcet et le calcul intégral. In R. Rashed, ed., Sciences à l'époque de la Révolution française, Blanchard, Paris, 1988, pp. 85-147.

E. Giusti (1983). Analisi matematica. Boringhieri, Torino, 1983, 2 volumes.

J.-V. Grabiner (1974). Is mathematical truth time-dependent? The American Mathematical Monthly, 81, 1974, pp. 354365.

J. V. Grabiner (1981a). The Origins of Cauchy's Rigorous Calculus. M.I.T. Press, Cambridge (Mass.), 1981.

J.-V. Grabiner (1981b). Changing attitudes towards mathematical rigor: Lagrange and analysis in the eighteenth and nineteenth centuries. In H. N. Jahnke and M. Otte, ed., Epistemological and Social Problems of the Sciences in the Early Nineteenth Century, D. Reidel P. C., Dordrecht, Boston, London, 1981, pp. 311-330.

J. V. Grabiner (1990). The Calculus as Algebra. J. L. Lagrange, 1736-1813. Garland Publishing, New York and London, 1990. Reprint of Grabiner's $1966 \mathrm{PhD}$ theis.

I. Grattan-Guinness (1970). The Development of Foundations of Mathematical Analysis from Euler to Riemann. MIT Press, Cambridge (Mass.), London, 1970, 3 volumes.

I. Grattan-Guinness (1990). Convolutions in French Mathematics, 1800-1840. Birkhāuser, Basel, Boston, Berlin, 1990, 3 volumes.

J. Gregory (1667). Vera circuli et hyperbolacequadratura […], ex Typographia Iacobi de Cadorinis, Patavii, 1667.

G. Gusdorf (1971). Les principes de la pensée au siècle des lumières, Payot, Paris, 1971.

M. Hallett (2008). Reflections on the purity of method in Hilbert's Grundlagen der Geometrie. In Mancosu (2008), pp. 198-255.

D. Hilbert (1898). Grundlagen der geometrie. In Festschrift zur Feier der Enthüllung des Gauss-Weber-Denkmals in Göttingen, B. G. Teubner, Leipzig, 1899, pp. 3-92.

G.-S. Klügel (1800). Erläuterungen über den beweis des polynomischen lehrsatzes von dem verfasser des beweises. Sammlung combinatorisch-analytischer Abhandlungen, 2, 1800, pp. 145-154. 
S.-F. Lacroix (1800). Traité des différences et des séries: faisant suite au Traité du calcul différentiel et du calcul intégral. Duprat, Paris, 1800, 2 volumes.

S.-F. Lacroix (1810-1819). Traité du calcul différentiel et du calcul intégral. Courcier, Paris, 1810-1819, 3 volumes.

S.-F. Lacroix (1797-1798). Traité du calcul différentiel et du calcul intégral. Duprat, Paris, ans. V-VI: 1797-1798, 2 volumes.

J.-L. Lagrange (1761). Essai d'une nouvelle méthode pour déterminer les maxima et les minima des formules intégrales indéfinies. Mélanges de Philosophie et de Mathématiques de la Soc. Roy. de Turin, 2, 1760-1761, 2nd part, pp. 173-95. Also in Lagrange (EUVRES), vol. I, pp. 335-362.

J.-L. Lagrange (1772). Sur une nouvelle espéce de calcul relatif à la différentiation et à l'intégration des quantités variables. Nouv. Mem. Acad. Roy Sci. Bell. Lett. [Berlin], 1772 (publied in 1774), pp. 185-221. Also in Lagrange (EUVRES), vol. III, pp. 441-476.

J.-L. Lagrange (1788). Méchanique analytique. Chez La Veuve Desaint, Paris, 1788.

J.-L. Lagrange (1797). Théorie des fonctions analytiques [...]. Impr. de la République, Paris, Prairial an V (May-June 1797).

J.-L. Lagrange (1798). De la résolution des équations numériques de tous les degrés. Duprat, Paris, an VI (1798).

J.-L. Lagrange (1799). Discours sur l'objet de la théorie des fonctions analytiques. Journal de l'École Polytechnique, 2 (6th cahier), Thermidor an VII (July-August 1799), pp. 232-235. Also in Lagrange (CEUVRES), vol. VII, pp. $325-328$.

J.-L. Lagrange (1801). Sur le calcul des fonctions. Impr. du Cercle Social, 1801. Vol. X (Leçons) of Ecole Normale III (NE); reprinted with slight revisions as the 12th cahier of the Journal de l'École Polytecnique, Thermidor, an XII (July-August 1804). References are relative to this last edition.

J.-L. Lagrange (1806). Leçons sur le calcul des fonctions, nouvelle édition revue, corrigée et augmentée par l'auteur. Courcier, Paris, 1806. Also in Lagrange (CEUVRES), vol. X.

J.-L. Lagrange (1808). Traité de la résolution des équations numériques de tous les degrés. Avec des Notes sur plusieurs points de la Théorie des équations algébriques. Courcier, Paris, 1808. Also in Lagrange (EUVRES), vol. VIII.

J.-L. Lagrange (1811-1815). Mécanique analytique. Courcier, Paris, 1811-1815, 2 volumes. Also in Lagrange (CEUVRS), volumes XI-XII.

J.-L. Lagrange (1813). Théorie des fonctions analytiques. Courcier, Paris, 1813. Also in Lagrange (EUVRES), vol. IX.

J.-L. Lagrange (EUVRES). Euvres de Lagrange, ed. by M. J.-A. Serret [et G. Darboux], Gauthier-Villars, Paris, 18671892,14 volumes.

E. Laguerre (1878-1879). Sur l'intégrale $\int_{z}^{\infty} \frac{e^{-x}}{x} d x$. Bulletin de la Société mathématique de France, 7, 1878-1879, pp. 7281.

P.-S. Laplace (1812). Théorie analytique des probabilités. Courcier, Paris, 1812.

P. Mancosu, ed. (2008) The Philosophy of Mathematical Practice. Clarendon Press, Oxford, 2008.

A. F. Monna (1972). The concept of function in the 19th and 20th centuries [… Archive for History of Exact Sciences, 9, 1972, pp. 57-84.

J. L. Ovaert (1976). La thèse de Lagrange et la transformation de l'analyse. In C. Houzel, L. L. Ovaert, P. Raymond, J.-L. Sansuc, eds., Philosophie et calcul de l'infini, Maspero, Paris, 1976, pp. 157-222.

M. Panza (1985). Il manoscritto del 1789 di Arbogast sui principi del calcolo differenziale e integrale. Rivista di storia della scienza, 2, 1985, pp. 123-157.

M. Panza (1991-1992). The analytical foundation of mechanics of discrete systems in Lagrange's théorie des fonctions analytiques, compared with Lagrange's earlier treatments of this topics. Historia Scientiarum, 44, 1991, pp. 87-132, and 45, 1992, pp. 181-212.

M. Panza (1992). La forma della quantità. Analisi algebrica e analisi superiore: il problema dell'unità della matematica nel secolo dell'illuminismo, volume 38 and 39 of Cahiers d'historie et de philosophie des sciences, Paris, 1992.

M. Panza (1996). Concept of function, between quantity and form, in the eighteenth century. In N. Knoche H. N. Jahnke and M. Otte, eds., History of Mathematics and Education: Ideas and Experiences, Vandenhoeck \& Ruprecht, Göttingen, 1996, pp. 241-274.

M. Panza (2003). The origins of analytical mechanics in 18th century. In H. N. Jahnke, ed., A History of Analysis, American Mathematical Society and London Mathematical Society, 2003, pp. 137-153.

M. Panza (2005). Netwon et les origines de l'analyse: 1664-1666. Blanchard, Paris, 2005.

M. Panza (2007). Euler's Introductio in analysin infinitorum and the program of algebraic analysis: quantities, functions and numerical partitions. In R. Backer, edi., Euler Reconsidered. Tercentenary essays, Kendrick Press, Heber City (Utah), 2007, pp. 119-166.

G. Peano (1891). Sulla formula di Taylor. Atti della Reale Accademia delle Scienze di Torino, 27, 1891, pp. 40-46. Also in: G. Peano, Opere Scelte, Edizioni Cremonese, Roma, 1957-1959, 3 volumes, vol. 1, pp. 204-209.

M. Pensivy (1987-1988). Jalons historiquesn pour une épistémologie de la série infinie du binôme, Vol. 14 de Sciences et techniques en perscective, 1987-1988. 
L. Pepe (1986). Tre 'prime edizioni' ed un'introduzione della Théorie des fonctions analytiques di Lagrange. Bollettino di storia delle scienze matematiche, 6, 1986, pp. 17-44.

S.-D. Poisson (1805). Démonstration du théorème de Taylor. Correspondance de l'École Polytechnique, 1, 1804-1805, issue of 3rd Pluviôse an XIII (January-February 1805), pp. 52-55.

G. R. de Prony (1796). Notice sur un cours élémentaire d'analyse fait par Lagrange. Journal de l'École Polytechnique, 1, 1796, pp. 206-208.

J. Sebestik (1964). Bernard Bolzano et son mémoire sur le théorème fondamental de l'analyse. Revue d'histoire des sciences, 17, 1964, pp. 129-164. Containing a French Translation of Bolzano (1817).

C. Truesdell (1995). The Rational Mechanics od Flexible or Elastic Bodies, 1638-1788, Vol. IX of Euler (OPERA), 1995.

J. Vuillemin (1962). La philosophie de l'algèbre. P.U.F., Paris, 1962.

M. V. Wilkers F. R. S. (1990). Herschel, Peacock, Babbage and the development of the Cambridge curriculum. Notes and Records of the Royal Society of London, 44, 1990 pp. 205-219.

R. Woodhouse (1803). The Principles of Analytical Calculation. Cambridge Univ. Press, Cambridge, 1803.

C. Wright (2000). Neo-Fregean foundations for real analysis: Some reflections on Frege's constraint. Notre Dame Journal of Formal Logic, 41, 2000, pp. 317-334.

A. Youschkevitch (1976-1977). The concept of function up to the middle of the 19th century. Archive for History of Exact Sciences, 16, 1976-1977, pp. 37-85.

K. Zimmermann (1934). Arbogast als Mathematoker, und Historiker der Mathematik. Ruprecht-Karls-Universität, Heidelberg, 1934. Inaugural-Dissertation zur Erlangung der Doktorwürde der Hohen NaturalwissenschaftlichMathelatischen Fakultät. 